\title{
Numerical Periodic Normalization for Codim 2 Bifurcations of Limit Cycles: Computational Formulas, Numerical Implementation, and Examples*
}

\author{
V. De Witte ${ }^{\dagger}$, F. Della Rossa ${ }^{\ddagger}$, W. Govaerts ${ }^{\dagger}$, and Yu. A. Kuznetsov ${ }^{\S}$
}

\begin{abstract}
Explicit computational formulas for the coefficients of the periodic normal forms for codimension 2 (codim 2) bifurcations of limit cycles in generic autonomous ODEs are derived. All cases (except the weak resonances) with no more than three Floquet multipliers on the unit circle are covered. The resulting formulas are independent of the dimension of the phase space and involve solutions of certain boundary-value problems on the interval $[0, T]$, where $T$ is the period of the critical cycle, as well as multilinear functions from the Taylor expansion of the ODE right-hand side near the cycle. The formulas allow one to distinguish between various bifurcation scenarios near codim 2 bifurcations of limit cycles. Our formulation makes it possible to use robust numerical boundaryvalue algorithms based on orthogonal collocation, rather than shooting techniques, which greatly expands its applicability. The implementation is described in detail with numerical examples, where numerous codim 2 bifurcations of limit cycles are analyzed for the first time.
\end{abstract}

Key words. normal forms, limit cycles, bifurcations, codimension 2

AMS subject classifications. 34C20, 37G15, 37M20, 65L07

DOI. $10.1137 / 120874904$

1. Introduction. Isolated periodic orbits (limit cycles) of smooth differential equations

$$
\dot{u}=f(u, p), \quad u \in \mathbb{R}^{n}, p \in \mathbb{R}^{m},
$$

play an important role in applications. In generic systems of the form (1.1) depending on one control parameter (i.e., with $m=1$ ) a hyperbolic limit cycle exists for an open interval of parameter values $p$. At a boundary of such an interval, the limit cycle may become nonhyperbolic, so that a cycle limit point (saddle-node), or a period-doubling (flip), or a torus (NeimarkSacker) bifurcation occurs. In two-parameter generic systems (1.1) (i.e., with $m=2$ ) these local bifurcations happen at certain curves in the parameter plane. These curves of codimension 1 (codim 1) bifurcations can meet tangentially or intersect transversally at some codimension 2 (codim 2 ) points characterized by a double degeneracy of the limit cycle which play the role of organizing centers for local dynamics, i.e., near the critical cycle and for nearby

\footnotetext{
${ }^{*}$ Received by the editors April 25, 2012; accepted for publication (in revised form) by H. Osinga January 24, 2013; published electronically May 14, 2013.

http://www.siam.org/journals/siads/12-2/87490.html

${ }^{\dagger}$ Department of Applied Mathematics and Computer Science, Ghent University, Krijgslaan 281-S9, B-9000, Ghent, Belgium (Virginie.DeWitte@UGent.be, Willy.Govaerts@UGent.be).

${ }^{\ddagger}$ Dipartimento di Elettronica e Informazione, Politecnico di Milano, Via Ponzio 35/5, 20133 Milano, Italy (fabio.dellarossa@mail.polimi.it).

${ }^{\S}$ Department of Mathematics, Utrecht University, Budapestlaan 6, PO Box 80010, 3508 TA Utrecht, The Netherlands (I.A.Kouznetsov@uu.nl) and Department of Applied Mathematics, University of Twente, PO Box 217, 7500 AE Enschede, The Netherlands.
} 
parameter values. In some cases, such codim 2 bifurcations imply the appearance of nearby "chaotic motions."

The codim 2 bifurcations of limit cycles in generic systems (1.1) are well understood with the help of the corresponding Poincaré maps and their normal forms (see, for example, $[24,2,4,3,29,20])$. However, applications of these results to the analysis of concrete systems (1.1) are exceptional, since they require accurate higher-order derivatives of the Poincaré map which are hardly available numerically [42, 22, 23, 32].

We note that there exists software, e.g., CAPD [9] and TIDES [1, 6], that allows us to compute up to any precision level the solution of an ODE using a Taylor series method in a variable stepsize - variable order formulation. It can also compute, up to any order, the partial derivatives of the solution with respect to the initial conditions. When applied to compute a periodic orbit by a shooting method, this will also provide the derivatives of the Poincaré map.

Though this is a valuable approach in some individual cases, it is difficult to use in a continuation context. Also, the shooting method does not have the high-order convergence properties of the method of approximation by piecewise polynomials with collocation in the Gauss points that is routinely used in the standard software such as AUTO [17], CONTENT [31], and MATCONT [15, 14]. Moreover, the number of derivatives of the Poincaré map to be computed is $O\left(n^{k}\right)$ if derivatives up to order $k$ are needed (in several cases $k=5$ ). Even for moderate values of $n$ this involves a great deal of unnecessary work since in our situation the normal form itself is known in advance and we need only compute its coefficients. We will show that this can be done without computing the derivatives of the Poincaré map.

Indeed, recently an alternative numerical method to analyze codim 1 limit cycle bifurcations has been developed and implemented in [30]. It is based on the periodic normalization proposed in $[19,25,26]$ and completely avoids the numerical computation of Poincaré maps and their derivatives. Instead, the computation of the normal form coeffcients is reduced to solving certain linear boundary-value problems (BVPs), where only the partial derivatives of the right-hand side (RHS) of (1.1) are used, and evaluating certain integrals. In our implementation in MATCONT, we discretize these BVPs by orthogonal collocation with piecewise-polynomial functions. Note that all appearing integrals can also be easily computed using this discretization.

Analytical studies of bifurcation phenomena and their normal forms are sometimes supported by numerical computations, e.g., in $[37,8]$. In such studies, curves of codim 1 bifurcations of limit cycles are computed (often with AUTO [17]), and codim 2 bifurcations are detected. However, since normal form coeffcients are not computed, the results cannot be compared to ours.

In the present paper, we apply the approach developed in [30] to codim 2 bifurcations of limit cycles. We therefore advise the reader to first get acquainted with [30]. It should be noted that already in [10] normal forms for some codim 2 bifurcations of cycles in (1.1) were derived, while [25] contains the periodic normal forms for many codim 2 bifurcations of cycles as well as a general normalization technique applicable at any codimension. However, in neither of these publications are explicit formulas for the normal form coefficients given in a form suitable for numerical implementation. The derivation of such formulas is the primary contribution of this paper. We also report full details of their implementation and 
give numerous numerical examples.

The paper is organized as follows. In section 2 we fix notation, list the periodic normal forms for codim 2 bifurcations of limit cycles in generic ODEs, and clarify the relationships between the periodic normal forms and bifurcations of the Poincaré maps. Note that we exclude the weakly resonant cases since traditionally, weak resonances are not included in the list of codim 2 bifurcations (see [3, page 48]) but are considered as generic codim 1 Neimark-Sacker bifurcations, where only one invariant torus appears. We follow this tradition. The birth of this torus is determined by the first Lyapunov coefficient that we do compute. However, Arnol'd also noticed that it is more natural to describe such bifurcations in twoparameter families (see pages 43 and 53 in [3]), where phase locking tongues appear. ${ }^{1}$ In section 3 we derive explicit formulas to compute the critical normal form coefficients for these bifurcations, which we order by the dimension $n_{c}$ of the cycle center manifold (i.e., the total number of critical multipliers with $|\mu|=1$ including the trivial multiplier 1). We restrict our attention to the cases $n_{c}=2$ and 3 but do not detect and thus do not compute normal forms for weak resonances. In this paper we study the resulting eight cases for which $n_{c}=2$ or 3. The formulas for the normal form coefficients for these bifurcations are independent of the dimension of the phase space and involve solutions of certain BVPs on the interval $[0, T]$, where $T$ is the period of the critical cycle, as well as multilinear functions from the Taylor expansion of the RHS of (1.1) near the cycle. In section 4 we give the explicit formulas for all needed functions and coefficients, but rescaled to the interval $[0,1]$, and discuss their implementation in MATCONT. In section 5, we focus on four examples which contain all eight codim 2 bifurcations of limit cycles with two- and three-dimensional center manifolds. We discuss in detail which type of codim 2 bifurcation occurs in each case and check the correspondence with the value/sign of the normal form coefficients.

We also provide some supplementary online material. In Appendix A of these supplementary files, we derive all necessary periodic normal forms based on [25, 26], where suggestions were made for the derivation in all codim 2 cases, except the fold-flip, but details were missing. In Appendix B some results on differential-difference operators used in section 4 are formulated. Appendix $\mathrm{C}$ contains a list of all needed functions and normal form coefficients in the considered eight cases. A tutorial is added as Appendix D, which allows the interested reader to do all the computations that led to the bifurcation diagrams reported in Figures 1 and 3.

2. Periodic normal forms on the center manifold. We write (1.1) at the critical parameter values as

$$
\dot{u}=F(u),
$$

and suppose that there is a limit cycle $\Gamma$ corresponding to a periodic solution $u_{0}(t)=u_{0}(t+T)$, where $T>0$ is its (minimal) period. Develop $F\left(u_{0}(t)+v\right)$ into the Taylor series

$$
\begin{aligned}
F\left(u_{0}(t)+v(t)\right) & =F\left(u_{0}(t)\right)+A(t) v(t)+\frac{1}{2} B(t ; v(t), v(t))+\frac{1}{3 !} C(t ; v(t), v(t), v(t)) \\
& +\frac{1}{4 !} D(t ; v(t), v(t), v(t), v(t))+\frac{1}{5 !} E(t ; v(t), v(t), v(t), v(t), v(t))+O\left(\|v\|^{6}\right),
\end{aligned}
$$

\footnotetext{
${ }^{1}$ The truncated critical normal form for a weak resonance depends on the order $q$ of the resonance, and its principle part is given by $\dot{z}=i \omega z+A z|z|^{2}+B \bar{z}^{q-1}$, where $\Re(A)$ determines the first Lyapunov coefficient but $B$ has very little dynamical meaning $(|B|$ linearly scales the width of the tongue [26]).
} 
where $A(t) v=F_{u}\left(u_{0}(t)\right) v, B\left(t ; v_{1}, v_{2}\right)=F_{\text {uu }}\left(u_{0}(t)\right)\left[v_{1}, v_{2}\right], C\left(t ; v_{1}, v_{2}, v_{3}\right)=F_{\text {uиu }}\left(u_{0}(t)\right)\left[v_{1}, v_{2}\right.$, $\left.v_{3}\right]$, etc. The multilinear forms $A, B, C, D$, and $E$ are periodic in $t$ with period $T$.

Consider the initial-value problem for the fundamental matrix solution $Y(t)$, namely,

$$
\frac{d Y}{d t}=A(t) Y, \quad Y(0)=I_{n},
$$

where $I_{n}$ is the $n \times n$ identity matrix. The eigenvalues of the monodromy matrix $M=Y(T)$ are called (Floquet) multipliers of the limit cycle. The multipliers with $|\mu|=1$ are called critical. There is always a "trivial" critical multiplier $\mu_{n}=1$. We denote the total number of critical multipliers (counting multiplicity) by $n_{c}$ and assume that the limit cycle is nonhyperbolic, i.e., $n_{c}>1$. In this case, there exists an invariant $n_{c^{-}}$-dimensional critical center manifold $W^{c}(\Gamma) \subset \mathbb{R}^{n}$ near $\Gamma$.

It is well known $[2,29]$ that in generic two-parameter systems such as (1.1) only 11 codim 2 local bifurcations of limit cycles occur. In this paper we consider eight of these cases, namely, those for which the dimension of the center manifold equals 2 or 3 (excluding the weak resonances). To describe the normal forms of generic (2.1) on the critical center manifold $W^{c}(\Gamma)$ for these codim 2 cases, we parameterize $W^{c}(\Gamma)$ near $\Gamma$ by $(\tau, \xi)$, where $\tau \in[0, k T]$ for $k \in\{1,2,3,4\}$ is a cyclic coordinate, and $\xi$ is a real or complex transverse coordinate, depending on the bifurcation. Note that $t$ represents the time of the dynamical system. It follows from [25] that it is possible to select the $\tau$ - and $\xi$-coordinates so that the restriction of (2.1) to the corresponding critical center manifold $W^{c}(\Gamma)$ with $n_{c}=2$ or $n_{c}=3$ will have a periodic normal form. Each normal form can be written as

$$
\left\{\begin{array}{l}
\frac{d \tau}{d t}=1+p(\xi)+r(\tau, \xi), \\
\frac{d \xi}{d t}=P(\xi)+R(\tau, \xi),
\end{array}\right.
$$

where $p$ and $P$ are polynomials in $\xi$ of some degree $N$ without constant terms given in Table 1 , while $r$ and $R$ are smooth $O\left(\|\xi\|^{N+1}\right)$-functions which are $k T$-periodic in $\tau$. We stress that the normal forms from Table 1 are valid for generic systems. In particular, it is assumed that a multiple critical eigenvalue of the monodromy matrix $M$ (when present) is not semisimple and that the corresponding Jordan chain has maximal length.

After a time reparameterization, (2.4) can be rewritten as

$$
\left\{\begin{array}{l}
\frac{d \tau}{d t}=1, \\
\frac{d \xi}{d t}=\tilde{P}(\xi)+\tilde{R}(\tau, \xi),
\end{array}\right.
$$

where $\tilde{P}$ and $\tilde{R}$ have the same properties as $P$ and $R$. The equation for $\xi$ will then become the nonautonomous system

$$
\frac{d \xi}{d t}=\tilde{P}(\xi)+\tilde{R}(t, \xi)
$$

with the $k T$-periodic in $t$ RHS. The $k T$-shift along orbits of the resulting autonomous truncated system,

$$
\dot{\xi}=\tilde{P}(\xi),
$$


Table 1

Truncated critical periodic normal forms with two- and three-dimensional center manifold.

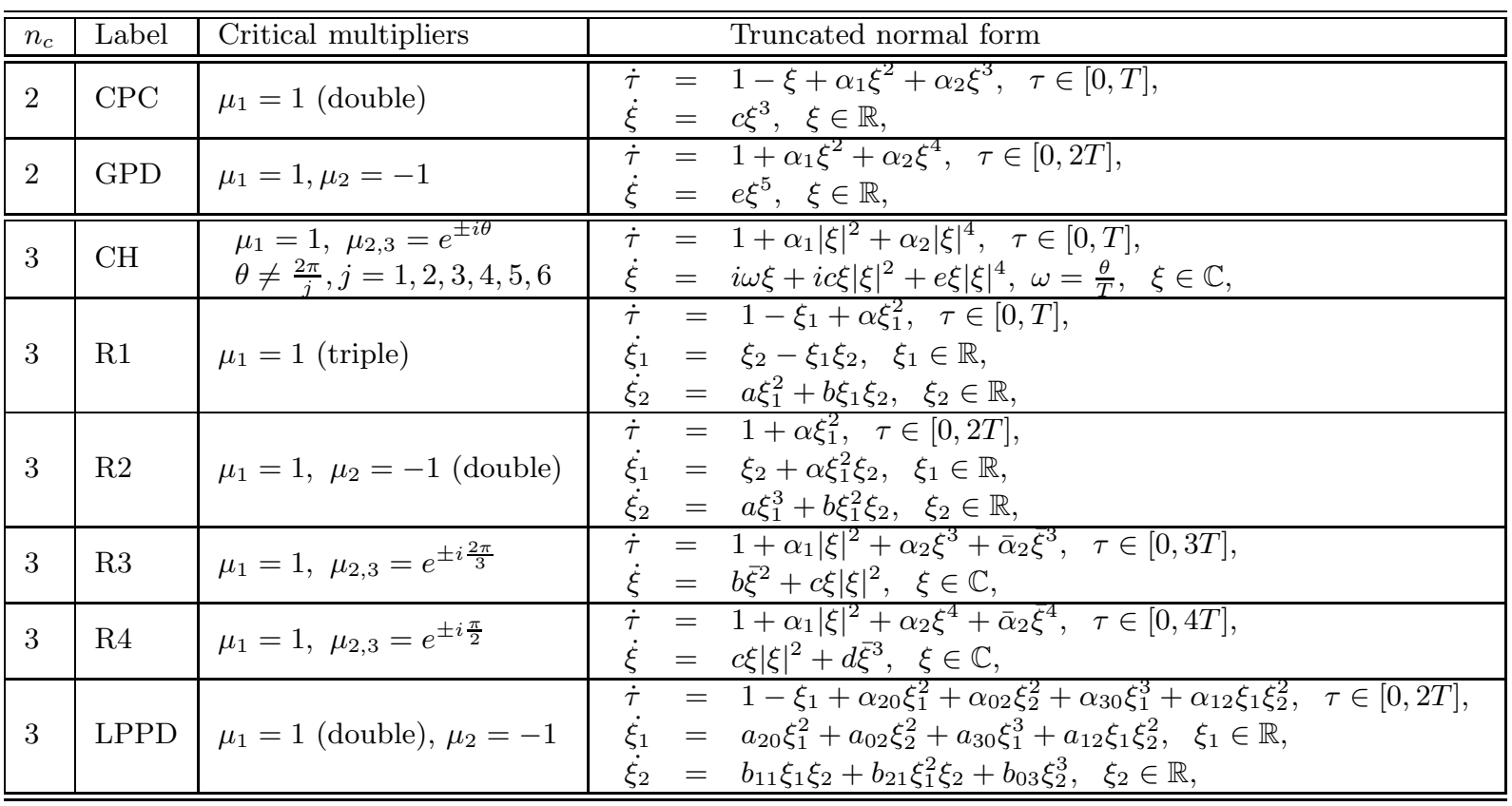

will approximate the $k$ th iterate of the Poincaré map associated with the limit cycle and restricted to the center manifold, in appropriate coordinates. Notice that the RHS of (2.6) has the same terms as the corresponding equation in the Iooss normal form in [25].

This construction can be carried out to parameter-dependent systems. In appropriate coordinates, a canonical unfolding of (2.6) will approximate the restricted Poincaré map of the generic two-parameter system (1.1) [25]. In what follows, we refer to the bifurcation diagrams of the unfoldings given in [29]. The complete overview of all bifurcation diagrams with phase portraits can be found in the arXiv paper [13].

\subsection{Bifurcations with two critical eigenvalues.}

2.1.1. Cusp point of cycles (CPC) bifurcation. In this case, (2.6) takes the form

$$
\dot{\xi}=c \xi^{3}, \quad \xi \in \mathbb{R},
$$

and the $T$-shift along its orbits approximates the restricted Poincaré map associated with the critical limit cycle. The canonical two-parameter unfolding of (2.7) is

$$
\dot{\xi}=\beta_{1}+\beta_{2} \xi+c \xi^{3},
$$

provided $c \neq 0$. Equilibria of these equations correspond to fixed points of the Poincaré maps, i.e., cycles in (1.1). When two equilibria collide at a limit point bifurcation, a limit point of cycles (LPC) bifurcation occurs in (1.1). The bifurcation diagram of this equation is shown in Figure 8.3 in section 8.2 of [29] for the case $c<0$. On curves $T_{1}$ and $T_{2}$, which meet tangentially at the cusp point, two limit cycles collide and disappear. When detecting a CPC point, the output given by MATCONT is the normal form coefficient $c$. 
2.1.2. Generalized period-doubling (GPD) bifurcation. In this case, (2.6) reduces to

$$
\dot{\xi}=e \xi^{5}, \quad \xi \in \mathbb{R},
$$

and the $2 T$-shift along its orbits will approximate the second iterate of the restricted Poincaré map associated with the critical limit cycle. The canonical two-parameter unfolding of (2.8) when $e \neq 0$ is

$$
\dot{\xi}=\beta_{1} \xi+\beta_{2} \xi^{3}+e \xi^{5} .
$$

The equilibrium $\xi=0$ of this equation corresponds to the fixed point of the Poincaré map, while symmetric nonzero equilibria of this equation correspond to its 2-cycles. Thus, a pitchfork bifurcation in this equation will describe a period-doubling bifurcation of a limit cycle in (1.1). If $e<0$, we obtain the bifurcation diagram reported in Figure 9.3 in [29], in which the limit point curve of the period-doubled limit cycles $T^{(2)}$ is tangent to the subcritical perioddoubling branch labeled as $F_{-}^{(1)}$. If $e>0$, we are in the opposite situation. The output given by MATCONT is the normal form coefficient $e$.

\subsection{Bifurcations with three critical eigenvalues.}

2.2.1. Chenciner $(\mathrm{CH})$ bifurcation. In this case, (2.6) becomes

$$
\dot{\xi}=i \omega \xi+i\left(c-\alpha_{1} \omega\right) \xi|\xi|^{2}+\left(e-i\left(\alpha_{1} c-\alpha_{1}^{2} \omega+\alpha_{2} \omega\right)\right) \xi|\xi|^{4},
$$

and the $T$-shift along its orbits will approximate the restricted Poincare map associated with the critical limit cycle. The canonical two-parameter unfolding of (2.9) is locally topologically equivalent to the normal form for the degenerate Hopf (Bautin) bifurcation

$$
\dot{\xi}=\left(\beta_{1}+i \omega\right) \xi+\beta_{2} \xi|\xi|^{2}+\Re(e) \xi|\xi|^{4},
$$

provided $\Re(e) \neq 0$. The trivial equilibrium $\xi=0$ corresponds to the bifurcating cycle in (1.1), while limit cycles in the $(\Re(\xi), \Im(\xi))$-plane correspond to closed invariant curves of the approximate Poincaré map, i.e., approximate invariant tori in (1.1). Note that actual invariant sets of (1.1) can be close to tori but have a much more complicated structure. The Hopf bifurcation will correspond to the Neimark-Sacker bifurcation, while the LPC at which two limit cycles collide and disappear will be substituted by a complicated bifurcation set where an "annihilation" of two closed invariant curves occurs. This set, however, is close to the LPC curve, which therefore will be referred to as the "limit point of tori curve."

The sign of $\Re(e)$ determines the bifurcation scenario: $\Re(e)<0$ corresponds with a stable critical limit cycle and $\Re(e)>0$ with an unstable critical limit cycle. When $\Re(e)<0$ the outer invariant curve is stable and the limit point of tori curve is tangent to the subcritical Neimark-Sacker branch, as shown in Figure 9.5 of [29]. When $\Re(e)>0$ the outer invariant curve is unstable and the limit point of tori curve is tangent to the supercritical NeimarkSacker branch. The output given by MATCONT is $\Re(e)$.

2.2.2. Strong resonance $1: 1$ (R1) bifurcation. In this case, (2.6) has the form

$$
\left\{\begin{array}{l}
\dot{\xi}_{1}=\xi_{2} \\
\dot{\xi}_{2}=a \xi_{1}^{2}+b \xi_{1} \xi_{2}
\end{array}\right.
$$


where it is assumed that $a b \neq 0$. The $T$-shift along orbits of this system will approximate the restricted Poincaré map associated with the critical limit cycle. The canonical two-parameter unfolding of (2.10) is given by the Bogdanov normal form

$$
\left\{\begin{array}{l}
\dot{\xi}_{1}=\xi_{2} \\
\dot{\xi}_{2}=\beta_{1}+\beta_{2} \xi_{1}+a \xi_{1}^{2}+b \xi_{1} \xi_{2}
\end{array}\right.
$$

with bifurcation diagrams depending on the sign of the product $a b$. Equilibria of this system correspond to fixed points of the Poincaré map, i.e., to cycles of (1.1), while its limit cycles approximate closed invariant curves of the map, i.e., invariant tori of (1.1). The Hopf bifurcation will thus correspond to the Neimark-Sacker bifurcation. In particular, as shown in Figure 8.8 of [29], if the two coefficients have different signs, the Neimark-Sacker curve $H$ is supercritical, while in the other case it is subcritical. The saddle homoclinic bifurcation in the Bogdanov normal form will correspond to a complicated sequence of bifurcations through which the torus self-destructs near a homoclinic tangle. The output given by MATCONT is the product of the coefficients $a$ and $b$.

2.2.3. Strong resonance $1: 2(\mathrm{R} 2)$ bifurcation. If we reparameterize time, (2.6) takes the form

$$
\left\{\begin{array}{l}
\dot{\xi}_{1}=\xi_{2} \\
\dot{\xi}_{2}=a \xi_{1}^{3}+b \xi_{1}^{2} \xi_{2}
\end{array}\right.
$$

The 2T-shift along its orbits will approximate the second iterate of the restricted Poincaré map associated with the critical limit cycle. The canonical two-parameter unfolding of (2.11) when $a b \neq 0$ is

$$
\left\{\begin{array}{l}
\dot{\xi}_{1}=\xi_{2}, \\
\dot{\xi}_{2}=\beta_{1} \xi_{1}+\beta_{2} \xi_{2}+a \xi_{1}^{3}+b \xi_{1}^{2} \xi_{2} .
\end{array}\right.
$$

We have four different bifurcation diagrams, determined by the signs of the coefficients. Those with negative $b$ are reported in Figure 9.9 of [29], where $a>0$, and Figure 9.10 of [29], where $a<0$. The other two cases can be obtained by reversing the arrows of the phase portraits and making a vertical flip both of the state portraits and of the bifurcation diagrams. The trivial equilibrium $\xi=0$ corresponds to the fixed point of the restricted Poincaré map, i.e., the bifurcating cycle of (1.1), while the nontrivial equilibria are the fixed points of the second iterate of the Poincaré map and correspond to one doubled cycle in (1.1). Thus, a pitchfork bifurcation implies the period-doubling bifurcation, and a Hopf bifurcation gives a NeimarkSacker bifurcation that generates an invariant torus. More complicated invariant sets and bifurcations are also possible. The primary Neimark-Sacker curve $H^{(1)}$ is supercritical (with negative normal form coefficient) if our coefficient $b$ is negative and subcritical otherwise. Moreover, if $a<0$, a secondary Neimark-Sacker curve $H^{(2)}$ is rooted at the 1:2 resonance point with opposite criticality of the primary one. The output given by MATCONT is $(a, b)$.

2.2.4. Strong resonance $1: 3$ (R3) bifurcation. In this case, (2.6) takes the form

$$
\dot{\xi}=b \bar{\xi}^{2}+c \xi|\xi|^{2}, \quad \xi \in \mathbb{C} .
$$


The 3T-shift along its orbits will approximate the third iterate of the restricted Poincaré map associated with the critical limit cycle. The canonical two-parameter unfolding of (2.12) when $b \neq 0$ and $\Re(c) \neq 0$ is

$$
\dot{\xi}=\left(\beta_{1}+i \beta_{2}\right) \xi+b \bar{\xi}^{2}+c \xi|\xi|^{2} .
$$

Its trivial equilibrium corresponds to the bifurcating limit cycle, while three nontrivial equilibria correspond to fixed points of the third iterate of the Poincaré map, i.e., the cycle in (1.1) with triple period. Moreover, a limit cycle in the $(\Re(\xi), \Im(\xi))$-plane approximates a closed invariant curve of the Poincaré map, i.e., an invariant torus in (1.1). The usual remark about the nature of the approximation is applicable here, but a Hopf bifurcation corresponds to the Neimark-Sacker one. As can be seen in Figure 9.12 of [29], if $\Re(c)<0$ the Neimark-Sacker bifurcation at the curve $N$ is supercritical (with negative normal form coefficient), while in the other case it is subcritical. The output given by MATCONT is $(b, \Re(c))$.

2.2.5. Strong resonance 1:4 (R4) bifurcation. Here (2.6) has the form

$$
\dot{\xi}=c \xi|\xi|^{2}+d \bar{\xi}^{3}, \quad \xi \in \mathbb{C} .
$$

The 4T-shift along its orbits will approximate the fourth iterate of the restricted Poincaré map associated with the critical limit cycle. The canonical two-parameter unfolding of (2.13) when the complex product $c d \neq 0$ is

$$
\dot{\xi}=\left(\beta_{1}+i \beta_{2}\right) \xi+c \xi|\xi|^{2}+d \bar{\xi}^{3},
$$

and its equilibria, cycles, and their bifurcations have the standard interpretations in terms of the original system (1.1). In particular, nonzero equilibria correspond to the fixed points of the fourth iterate of the Poincaré map, i.e., one cycle of approximate period-4T in (1.1). The bifurcation diagram of the unfolding depends on the complex number

$$
A=\frac{c}{|d|}
$$

(see $[28,29]$ and references therein). Many topologically different bifurcation diagrams can be found near the 1:4 resonance point. The analysis, if one excludes higher codimension situations, can be reduced to 22 different cases. First, analyzing the unfolding, one can divide the $A$-plane into two big regions: in the semiplane $\Re(A)<0$ the primary Neimark-Sacker bifurcation is supercritical, and in the semiplane $\Re(A)>0$ it is subcritical. What happens in the semiplane $\Re(A)>0$ can therefore be obtained by inverting the direction of the vector fields. We can further reduce the analysis to the third quadrant of the $A$-plane, since the 12 possible cases are topologically equivalent paired through the transformation $\xi \mapsto \bar{\xi}$. The different regions are shown in Figure 9.14 of section 9.5.5 in [29], in which some curves are computed numerically.

The output given by MATCONT is $(A, d)$.

2.2.6. Fold-flip (LPPD) bifurcation. In this final case, (2.6) has the form

$$
\left\{\begin{array}{l}
\dot{\xi}_{1}=a_{20} \xi_{1}^{2}+a_{02} \xi_{2}^{2}+\left(a_{30}+a_{20}\right) \xi_{1}^{3}+\left(a_{12}+a_{02}\right) \xi_{1} \xi_{2}^{2}, \\
\dot{\xi}_{2}=b_{11} \xi_{1} \xi_{2}+\left(b_{21}+b_{11}\right) \xi_{1}^{2} \xi_{2}+b_{03} \xi_{2}^{3} .
\end{array}\right.
$$


The $2 T$-shift along its orbits will approximate the second iterate of the restricted Poincaré map associated with the critical limit cycle. If $b_{11} \neq 0$, the canonical unfolding of (2.14) is provided by

$$
\left\{\begin{array}{l}
\dot{\xi}_{1}=\beta_{1}+\beta_{2} \xi_{1}+a_{20} \xi_{1}^{2}+a_{02} \xi_{2}^{2}+\left(a_{30}+a_{20}\right) \xi_{1}^{3}+\left(a_{12}+a_{02}\right) \xi_{1} \xi_{2}^{2} \\
\dot{\xi}_{2}=b_{11} \xi_{1} \xi_{2}+\left(b_{21}+b_{11}\right) \xi_{1}^{2} \xi_{2}+b_{03} \xi_{2}^{3}
\end{array}\right.
$$

Its equilibria and cycles have standard interpretations in terms of the original system (1.1). In particular, equilibria with $\xi_{2} \neq 0$ correspond to a double-period cycle, while their Hopf bifurcation represents a Neimark-Sacker (torus) bifurcation of this cycle in (1.1). Bifurcations of limit cycles approximate torus bifurcations. The critical coefficients allow us to determine which bifurcation scenario takes place. In particular (see [29, 33] for more details), three additional nondegeneracy conditions are involved:

- if $a_{20} \neq 0$ there are two limit cycles which collide and disappear;

- if $a_{02} \neq 0$ the double-period limit cycle is born;

- if $a_{02} b_{11}<0$ a nondegenerate torus bifurcation occurs for the period-doubled cycle, with the Lyapunov coefficient that might differ by a positive factor from

$$
\begin{aligned}
C_{N S}= & -2 a_{20} b_{21} a_{02}+6 b_{03} a_{20}^{2}+\left(-2 a_{02} b_{21}-6 a_{20} a_{02}+2 a_{20} b_{03}-3 a_{02} a_{30}-a_{12} a_{20}\right) b_{11} \\
& +b_{11}^{2}\left(a_{12}-a_{02}\right),
\end{aligned}
$$

provided $C_{N S} \neq 0$.

In Figures 9.25-9.28 of [29] four possible scenarios are reported depending on the sign of the normal form coefficients. The output given by MATCONT is $\left(b_{11}, a_{20}, a_{02}, C_{N S}\right)$.

3. Computation of critical coefficients. Taking into account previous results, we can assume that a parameterization of the center manifold $W^{c}(\Gamma)$ has been selected so that the restriction of (2.1) to this manifold has one of the normal forms given in section 2 . We then apply the so-called homological equation approach [7]: the Taylor expansions of $T-, 2 T-$, $3 T$-, or $4 T$-periodic unknown functions involved in these parameterizations can be found by solving appropriate BVPs on $[0, T]$ so that $(2.1)$ restricted to $W^{c}(\Gamma)$ has the corresponding periodic normal form. The coefficients of the normal forms arise from the Fredholm solvability conditions for the BVPs as integrals of scalar products over $[0, T]$, involving nonlinear terms of (2.1) near the periodic solution $u_{0}$, as well as the critical (generalized) eigenfunctions and already known expansion terms of the center manifold. A significant improvement with respect to [30] is that we systematically use (anti)periodicity properties of individual terms in the Taylor expansions. Note that BVPs for the generalized eigenfunctions are set up under the same genericity assumptions on the Jordan structure of the monodromy matrix which are used in the derivation of the corresponding critical normal forms.

The coefficient functions in the Taylor expansion are usually unique up to the addition of a multiple of a known eigenfunction. This can be fixed by adding an integral condition. Among other things this leads to the fact that normal form coefficients are not unique, but implications for the underlying dynamical systems are independent of this. We also remark that the solvability of all the equations up to the maximal order of the normal form has to be checked. Finally, we note that the coefficients in the equation for the cyclic variable will be computed only when needed for the computation of other critical coefficients. 


\subsection{Bifurcations with a two-dimensional center manifold.}

3.1.1. Cusp point of cycles bifurcation. The two-dimensional critical center manifold $W^{c}(\Gamma)$ at the cusp point of cycles (CPC) bifurcation can be parameterized locally by $(\tau, \xi)$ as

$$
u=u_{0}(\tau)+\xi v(\tau)+H(\tau, \xi), \quad \tau \in[0, T], \xi \in \mathbb{R},
$$

where $H$ satisfies $H(T, \xi)=H(0, \xi)$ and has the Taylor expansion

$$
H(\tau, \xi)=\frac{1}{2} h_{2}(\tau) \xi^{2}+\frac{1}{6} h_{3}(\tau) \xi^{3}+O\left(\xi^{4}\right)
$$

with $h_{j}(T)=h_{j}(0)$ for $j=2,3$, while the generalized eigenfunction $v$ is defined (as a function of $\tau$ ) by

$$
\left\{\begin{aligned}
\dot{v}-A(\tau) v-F\left(u_{0}\right) & =0, \tau \in[0, T] \\
v(T)-v(0) & =0 \\
\int_{0}^{T}\left\langle v, F\left(u_{0}\right)\right\rangle d \tau & =0
\end{aligned}\right.
$$

where the dot denotes the derivative with respect to $\tau$ (this notation will be used throughout this section). The function $v$ exists due to Lemma 2 of [25]. Let $\varphi^{*}$ be a nontrivial solution of the adjoint eigenvalue problem

$$
\left\{\begin{array}{l}
\dot{\varphi}^{*}+A^{\mathrm{T}}(\tau) \varphi^{*}=0, \quad \tau \in[0, T] \\
\varphi^{*}(T)-\varphi^{*}(0)=0
\end{array}\right.
$$

and the generalized adjoint eigenfunction $v^{*}$ a solution of

$$
\left\{\begin{aligned}
\dot{v}^{*}+A^{\mathrm{T}}(\tau) v^{*}+\varphi^{*} & =0, \tau \in[0, T] \\
v^{*}(T)-v^{*}(0) & =0
\end{aligned}\right.
$$

which is now defined up to the addition of a multiple of $\varphi^{*}$. Note that the first equation of (3.3) implies

$$
\int_{0}^{T}\left\langle\varphi^{*}, F\left(u_{0}\right)\right\rangle d \tau=0
$$

for $\varphi^{*}$ satisfying (3.4). Moreover, due to spectral assumptions at the CPC point, we can also assume

$$
\int_{0}^{T}\left\langle\varphi^{*}, v\right\rangle d \tau=1
$$

Notice that this assumption gives us another normalization condition for free, since taking into account (3.3) and (3.5) we have

$$
\int_{0}^{T}\left\langle v^{*}, F\left(u_{0}\right)\right\rangle d \tau=\int_{0}^{T}\left\langle\varphi^{*}, v\right\rangle d \tau=1
$$


i.e., we have automatically normalized the eigenfunction of the adjoint problem with respect to the generalized eigenfunction of the original problem and the generalized eigenfunction of the adjoint problem with respect to the eigenfunction of the original problem. So $\varphi^{*}$ is the unique solution of the BVP

$$
\left\{\begin{aligned}
\dot{\varphi}^{*}+A^{\mathrm{T}}(\tau) \varphi^{*} & =0, \tau \in[0, T], \\
\varphi^{*}(T)-\varphi^{*}(0) & =0 \\
\int_{0}^{T}\left\langle\varphi^{*}, v\right\rangle d \tau-1 & =0
\end{aligned}\right.
$$

We still need an integral condition for the adjoint generalized eigenfunction $v^{*}$. In all cases, for the computation of an adjoint generalized eigenfunction we will require the inner product with an original eigenfunction to be zero. Here, the inner product with $v$ is appropriate. Therefore, we obtain

$$
\left\{\begin{aligned}
\dot{v}^{*}+A^{\mathrm{T}}(\tau) v^{*}+\varphi^{*} & =0, \quad \tau \in[0, T], \\
v^{*}(T)-v^{*}(0) & =0 \\
\int_{0}^{T}\left\langle v^{*}, v\right\rangle d \tau & =0 .
\end{aligned}\right.
$$

Now, we substitute (3.1) into (2.1), using (2.2), the CPC normal form in Table 1, and (3.2). This gives

$$
\begin{aligned}
& \dot{u}_{0}+\xi\left(\dot{v}-\dot{u}_{0}\right)+\xi^{2}\left(\alpha_{1} \dot{u}_{0}-\dot{v}+\frac{1}{2} h_{2}\right)+\xi^{3}\left(\alpha_{2} \dot{u}_{0}+\alpha_{1} \dot{v}-\frac{1}{2} \dot{h}_{2}+\frac{1}{6} \dot{h}_{3}+c v\right)+O\left(\xi^{4}\right) \\
=F\left(u_{0}\right)+ & \xi A(\tau) v+\frac{1}{2} \xi^{2}\left(A(\tau) h_{2}+B(\tau ; v, v)\right) \\
& +\frac{1}{6} \xi^{3}\left(A(\tau) h_{3}+3 B\left(\tau ; h_{2}, v\right)+C(\tau ; v, v, v)\right)+O\left(\xi^{4}\right) .
\end{aligned}
$$

Collecting the $\xi^{0}$-terms, we get the identity $\dot{u}_{0}=F\left(u_{0}\right)$, since $u_{0}$ is the periodic solution of (2.1). The $\xi^{1}$-terms provide another identity, namely, $\dot{v}-\dot{u}_{0}=A(\tau) v$, as stated in (3.3).

From collecting the $\xi^{2}$-terms we obtain an equation for $h_{2}$ :

$$
\dot{h}_{2}-A(\tau) h_{2}=B(\tau ; v, v)+2 \dot{v}-2 \alpha_{1} \dot{u}_{0} .
$$

The differential operator $\frac{d}{d \tau}-A(\tau)$ on the left-hand side is singular in the space of vectorfunctions on $[0, T]$ satisfying $h_{2}(T)=h_{2}(0)$, since $\dot{u}_{0}$ is in its kernel. Now, we project the left-hand side of (3.11) on the adjoint null-eigenfunction, i.e., we take the scalar product with $\varphi^{*}$ pointwise and integrate the result over $[0, T]$, to obtain

$$
\int_{0}^{T}\left\langle\varphi^{*},\left(\frac{d}{d \tau}-A(\tau)\right) h_{2}\right\rangle d \tau=-\int_{0}^{T}\left\langle\left(\frac{d}{d \tau}+A^{T}(\tau)\right) \varphi^{*}, h_{2}\right\rangle d \tau=0
$$

due to (3.4). Therefore, the projection of the RHS of (3.11) on $\varphi^{*}$ also has to vanish, which leads to the so-called Fredholm solvability condition:

$$
\int_{0}^{T}\left\langle\varphi^{*}, B(\tau ; v, v)+2 \dot{v}-2 \alpha_{1} \dot{u}_{0}\right\rangle d \tau=\int_{0}^{T}\left\langle\varphi^{*}, B(\tau ; v, v)+2 A(\tau) v\right\rangle d \tau=0
$$


Notice that this condition is actually trivially satisfied, due to the fact that we are at the CPC point, so that the second order normal form coefficient (see [30])

$$
b=\frac{1}{2} \int_{0}^{T}\left\langle\varphi^{*}, B(\tau ; v, v)+2 A(\tau) v\right\rangle d \tau
$$

vanishes. Hence (3.11) is solvable, independent of the value of $\alpha_{1}$. For any value of $\alpha_{1}$ we get an equation for $h_{2}$ to be solved in the space of vector-functions on $[0, T]$ satisfying $h_{2}(T)=h_{2}(0)$. Notice that if $h_{2}$ satisfies (3.11), $h_{2}+\varepsilon F\left(u_{0}\right)$ also satisfies (3.11), due to the fact that $F\left(u_{0}\right)=\dot{u}_{0}$. The orthogonality condition with $v^{*}$ determines the value of $\varepsilon$ such that we can define $h_{2}$ as the unique solution of

$$
\left\{\begin{aligned}
\dot{h}_{2}-A(\tau) h_{2}-B(\tau ; v, v)-2 A v-2 F\left(u_{0}\right)+2 \alpha_{1} F\left(u_{0}\right) & =0, \tau \in[0, T] \\
h_{2}(T)-h_{2}(0) & =0 \\
\int_{0}^{T}\left\langle v^{*}, h_{2}\right\rangle d \tau & =0
\end{aligned}\right.
$$

Collecting the $\xi^{3}$-terms we finally obtain an equation in $h_{3}$ which allows us to determine the normal form coefficient $c$ of the CPC normal form in Table 1, namely,

$$
\dot{h}_{3}-A(\tau) h_{3}=-6 \alpha_{2} \dot{u}_{0}-6 \alpha_{1} \dot{v}+3 \dot{h}_{2}-6 c v+3 B\left(\tau ; h_{2}, v\right)+C(\tau ; v, v, v) .
$$

The Fredholm solvability condition implies that

$$
\int_{0}^{T}\left\langle\varphi^{*},-6 \alpha_{2} \dot{u}_{0}-6 \alpha_{1} \dot{v}+3 \dot{h}_{2}-6 c v+3 B\left(\tau ; h_{2}, v\right)+C(\tau ; v, v, v)\right\rangle d \tau=0 .
$$

Using this equation together with (3.3), (3.7), and (3.6), we get the expression

$$
c=\frac{1}{6} \int_{0}^{T}\left\langle\varphi^{*},-6 \alpha_{1} A(\tau) v+3 A(\tau) h_{2}+3 B(\tau ; v, v)+6 A(\tau) v+3 B\left(\tau ; h_{2}, v\right)+C(\tau ; v, v, v)\right\rangle d \tau,
$$

where $v$ and $\varphi^{*}$ are defined by (3.3) and (3.9), while $h_{2}$ satisfies (3.12).

Finally, let us prove that the choice of $\alpha_{1}$ does not influence the value of the critical normal form coefficient $c$. Indeed, two solutions $h_{2}$ corresponding to $\alpha_{1}^{(1)} \neq \alpha_{1}^{(2)}$ in (3.12) differ by $h_{2}^{(2)}-h_{2}^{(1)}=-2\left(\alpha_{1}^{(2)}-\alpha_{1}^{(1)}\right) v$, from which it follows that

$$
c^{(2)}-c^{(1)}=\left(\alpha_{1}^{(2)}-\alpha_{1}^{(1)}\right) \int_{0}^{T}\left\langle\varphi^{*},-2 A(\tau) v-B(\tau ; v, v)\right\rangle d \tau=\left(\alpha_{1}^{(1)}-\alpha_{1}^{(2)}\right) b=0,
$$

since $b=0$. So, for simplicity, we take $\alpha_{1}=0$, which further simplifies the expression for $c$. The critical coefficient $c$ in the periodic CPC normal form has thus been computed. The bifurcation is nondegenerate if $c \neq 0$. 
3.1.2. Generalized period-doubling bifurcation. The two-dimensional critical center manifold $W^{c}(\Gamma)$ at the generalized period-doubling (GPD) bifurcation can be parameterized locally by $(\tau, \xi)$ as

$$
u=u_{0}(\tau)+\xi v(\tau)+H(\tau, \xi), \quad \tau \in[0,2 T], \xi \in \mathbb{R},
$$

where the function $H$ satisfies $H(2 T, \xi)=H(0, \xi)$. It has the Taylor expansion

$$
H(\tau, \xi)=\frac{1}{2} h_{2}(\tau) \xi^{2}+\frac{1}{6} h_{3}(\tau) \xi^{3}+\frac{1}{24} h_{4}(\tau) \xi^{4}+\frac{1}{120} h_{5}(\tau) \xi^{5}+O\left(\xi^{6}\right),
$$

where $h_{j}(2 T)=h_{j}(0)$, while

$$
\left\{\begin{aligned}
\dot{v}-A(\tau) v & =0, \tau \in[0, T], \\
v(T)+v(0) & =0, \\
\int_{0}^{T}\langle v, v\rangle d \tau-1 & =0,
\end{aligned}\right.
$$

and

$$
v(\tau+T)=-v(\tau) \text { for } \tau \in[0, T] .
$$

The function $v$ exists due to Lemma 5 of [25].

The functions $h_{i}, i=2, \ldots, 5$, can be found by solving appropriate BVPs, assuming that (2.1) restricted to $W^{c}(\Gamma)$ has the periodic GPD normal form in Table 1. From (3.13) and (3.14) it follows that $h_{i}(\tau+T)=h_{i}(\tau)$ for $i$ even and $h_{i}(\tau+T)=-h_{i}(\tau)$ for $i$ odd, for $\tau \in[0, T]$. Indeed, since we are at the GPD point $u(\tau, \xi)=u(\tau+T,-\xi)$,

$$
\sum_{i} \frac{1}{i !} h_{i}(\tau) \xi^{i}=\sum_{i} \frac{1}{i !} h_{i}(\tau+T)(-1)^{i} \xi^{i}
$$

and thus

$$
h_{i}(\tau)=(-1)^{i} h_{i}(\tau+T),
$$

from which the statement follows. This makes it possible to restrict our considerations to the interval $[0, T]$ instead of $[0,2 T]$.

The coefficients $\alpha_{1}, \alpha_{2}$, and $e$ arise from the solvability conditions for the BVPs as integrals of scalar products over the interval $[0, T]$. Specifically, these scalar products involve among other things the terms up to the fifth order of (1.1) near the periodic solution $u_{0}$, the eigenfunction $v$, the adjoint eigenfunction $\varphi^{*}$ satisfying

$$
\left\{\begin{aligned}
\dot{\varphi}^{*}+A^{\mathrm{T}}(\tau) \varphi^{*} & =0, \quad \tau \in[0, T], \\
\varphi^{*}(T)-\varphi^{*}(0) & =0 \\
\int_{0}^{T}\left\langle\varphi^{*}, F\left(u_{0}\right)\right\rangle d \tau-1 & =0
\end{aligned}\right.
$$

and a similar adjoint eigenfunction $v^{*}$ satisfying

$$
\left\{\begin{aligned}
\dot{v}^{*}+A^{\mathrm{T}}(\tau) v^{*} & =0, \tau \in[0, T] \\
v^{*}(T)+v^{*}(0) & =0 \\
\int_{0}^{T}\left\langle v^{*}, v\right\rangle d \tau-1 & =0
\end{aligned}\right.
$$


To derive the normal form coefficient, we proceed as in section 3.1.1; namely, we substitute (3.13) into (2.1) and use the GPD normal form Table 1, (3.14), as well as (2.2).

Collecting the $\xi^{0}$ - and $\xi^{1}$-terms in the resulting equation gives the trivial identities, namely, $\dot{u}_{0}=F\left(u_{0}\right)$ and $\dot{v}=A(\tau) v$, if we take (3.15) into account.

By collecting the $\xi^{2}$-terms, we obtain the equation for $h_{2}$,

$$
\dot{h}_{2}-A(\tau) h_{2}=B(\tau ; v, v)-2 \alpha_{1} \dot{u}_{0},
$$

which is to be solved in the space of functions satisfying $h_{2}(T)=h_{2}(0)$. In this space, the differential operator $\frac{d}{d \tau}-A(\tau)$ is singular with null-function $\dot{u}_{0}$. Thus, the following Fredholm solvability condition has to be satisfied:

$$
\int_{0}^{T}\left\langle\varphi^{*}, B(\tau ; v, v)-2 \alpha_{1} \dot{u}_{0}\right\rangle d \tau=0
$$

This leads to the expression

$$
\alpha_{1}=\frac{1}{2} \int_{0}^{T}\left\langle\varphi^{*}, B(\tau ; v, v)\right\rangle d \tau
$$

where $v$ and $\varphi^{*}$ are defined by (3.15) and (3.16), respectively.

With $\alpha_{1}$ defined in this way, let $h_{2}$ be a solution of (3.18) in the space of functions satisfying $h_{2}(0)=h_{2}(T)$. Notice also here that if $h_{2}$ is a solution of (3.18), then also $h_{2}+\varepsilon_{1} F\left(u_{0}\right)$ satisfies (3.18), since $F\left(u_{0}\right)$ is in the kernel of the operator $\frac{d}{d \tau}-A(\tau)$. In order to obtain a unique solution (without projection on the null-eigenspace) we impose the following orthogonality condition which determines the value of $\varepsilon_{1}$ :

$$
\int_{0}^{T}\left\langle\varphi^{*}, h_{2}\right\rangle d \tau=0
$$

Thus $h_{2}$ is the unique solution of the BVP

$$
\left\{\begin{aligned}
\dot{h}_{2}-A(\tau) h_{2}-B(\tau ; v, v)+2 \alpha_{1} F\left(u_{0}\right) & =0, \\
h_{2}(T)-h_{2}(0) & =0 \\
\int_{0}^{T}\left\langle\varphi^{*}, h_{2}\right\rangle d \tau & =0 .
\end{aligned}\right.
$$

Collecting the $\xi^{3}$-terms, we get the equation for $h_{3}$,

$$
\dot{h}_{3}-A(\tau) h_{3}=C(\tau ; v, v, v)+3 B\left(\tau ; v, h_{2}\right)-6 \alpha_{1} \dot{v}
$$

which is to be solved in the space of functions satisfying $h_{3}(T)=-h_{3}(0)$. In this space the differential operator $\frac{d}{d \tau}-A(\tau)$ has a one-dimensional null-space, spanned by $v$, and (3.21) is solvable only if the RHS of this equation lies in the range of that operator. Using (3.15), we can rewrite the RHS as

$$
C(\tau ; v, v, v)+3 B\left(\tau ; v, h_{2}\right)-6 \alpha_{1} A(\tau) v
$$


Note that the Fredholm solvability condition

$$
\int_{0}^{T}\left\langle v^{*}, C(\tau ; v, v, v)+3 B\left(\tau ; v, h_{2}\right)-6 \alpha_{1} A(\tau) v\right\rangle d \tau=0
$$

is trivially satisfied due to the fact that we are in the GPD point, and so the cubic coefficient of the normal form (see [30])

$$
c=\frac{1}{3} \int_{0}^{T}\left\langle v^{*}, C(\tau ; v, v, v)+3 B\left(\tau ; v, h_{2}\right)-6 \alpha_{1} A(\tau) v\right\rangle d \tau
$$

vanishes. Since the RHS of (3.21) is in the range space of the operator $\frac{d}{d \tau}-A(\tau)$, we can solve the equation in order to find $h_{3}$ as the unique solution of the BVP

$$
\left\{\begin{aligned}
\dot{h}_{3}-A(\tau) h_{3}-C(\tau ; v, v, v)-3 B\left(\tau ; v, h_{2}\right)+6 \alpha_{1} A(\tau) v & =0, \tau \in[0, T], \\
h_{3}(T)+h_{3}(0) & =0, \\
\int_{0}^{T}\left\langle v^{*}, h_{3}\right\rangle d \tau & =0 .
\end{aligned}\right.
$$

By collecting the $\xi^{4}$-terms, we get the equation for $h_{4}$,

$$
\begin{aligned}
\dot{h}_{4}-A(\tau) h_{4}= & D(\tau ; v, v, v, v)+6 C\left(\tau ; v, v, h_{2}\right)+3 B\left(\tau ; h_{2}, h_{2}\right) \\
& +4 B\left(\tau ; v, h_{3}\right)-12 \alpha_{1} \dot{h}_{2}-24 \alpha_{2} \dot{u}_{0},
\end{aligned}
$$

which is to be solved in the space of functions satisfying $h_{4}(T)=h_{4}(0)$. The Fredholm solvability condition gives us the following expression for $\alpha_{2}$ :

$$
\alpha_{2}=\frac{1}{24} \int_{0}^{T}\left\langle\varphi^{*}, D(\tau ; v, v, v, v)+6 C\left(\tau ; v, v, h_{2}\right)+3 B\left(\tau ; h_{2}, h_{2}\right)+4 B\left(\tau ; v, h_{3}\right)-12 \alpha_{1} \dot{h}_{2}\right\rangle d \tau
$$

which by considering (3.18) can be simplified to

$$
\begin{aligned}
\alpha_{2}=\frac{1}{24} \int_{0}^{T} & \left\langle\varphi^{*}, D(\tau ; v, v, v, v)+6 C\left(\tau ; v, v, h_{2}\right)+3 B\left(\tau ; h_{2}, h_{2}\right)\right. \\
& \left.+4 B\left(\tau ; v, h_{3}\right)-12 \alpha_{1}\left(A(\tau) h_{2}+B(\tau ; v, v)\right)\right\rangle d \tau+\alpha_{1}^{2},
\end{aligned}
$$

where $\alpha_{1}$ is given by (3.19) and $h_{2}, h_{3}, v$, and $\varphi^{*}$ are the solutions of the BVPs (3.20), (3.23), (3.15), and (3.16), respectively.

Using this value of $\alpha_{2}$, we can find $h_{4}$ by solving

$$
\left\{\begin{aligned}
\dot{h}_{4}-A(\tau) h_{4}-D(\tau ; v, v, v, v)-6 C\left(\tau ; v, v, h_{2}\right)-3 B\left(\tau ; h_{2}, h_{2}\right) & \\
-4 B\left(\tau ; v, h_{3}\right)+12 \alpha_{1}\left(A(\tau) h_{2}+B(\tau ; v, v)-2 \alpha_{1} F\left(u_{0}\right)\right)+24 \alpha_{2} F\left(u_{0}\right) & =0, \tau \in[0, T], \\
h_{4}(T)-h_{4}(0) & =0 \\
\int_{0}^{T}\left\langle\varphi^{*}, h_{4}\right\rangle d \tau & =0
\end{aligned}\right.
$$

Finally, by collecting the $\xi^{5}$-terms, we get the equation for $h_{5}$,

$$
\begin{aligned}
\dot{h}_{5}-A(\tau) h_{5}= & E(\tau ; v, v, v, v, v)+10 D\left(\tau ; v, v, v, h_{2}\right)+15 C\left(\tau ; v, h_{2}, h_{2}\right)+10 C\left(\tau ; v, v, h_{3}\right) \\
& +10 B\left(\tau ; h_{2}, h_{3}\right)+5 B\left(\tau ; v, h_{4}\right)-120 \alpha_{2} \dot{v}-20 \alpha_{1} \dot{h}_{3}-120 e v,
\end{aligned}
$$


which has to be solved in the space of functions satisfying $h_{5}(T)=-h_{5}(0)$. Since the operator $\frac{d}{d \tau}-A(\tau)$ has a one-dimensional null-space, we can apply the Fredholm solvability condition to compute the parameter $e$ in the GPD normal form from Table 1. Using the normalization of (3.17), (3.23), and (3.22), we get

$$
\begin{gathered}
e=\frac{1}{120} \int_{0}^{T}\left\langle v^{*}, E(\tau ; v, v, v, v, v)+10 D\left(\tau ; v, v, v, h_{2}\right)+15 C\left(\tau ; v, h_{2}, h_{2}\right)+10 C\left(\tau ; v, v, h_{3}\right)\right. \\
\left.+10 B\left(\tau ; h_{2}, h_{3}\right)+5 B\left(\tau ; v, h_{4}\right)-120 \alpha_{2} A(\tau) v-20 \alpha_{1} A(\tau) h_{3}\right\rangle d \tau .
\end{gathered}
$$

If this quantity does not vanish, the codim 2 bifurcation is nondegenerate.

\subsection{Bifurcations with a three-dimensional center manifold.}

3.2.1. Chenciner bifurcation. The three-dimensional critical center manifold $W^{c}(\Gamma)$ at the Chenciner $(\mathrm{CH})$ bifurcation can be parameterized locally by $(\tau, \xi)$ as

$$
u=u_{0}(\tau)+\xi v(\tau)+\bar{\xi} \bar{v}(\tau)+H(\tau, \xi, \bar{\xi}), \quad \tau \in[0, T], \xi \in \mathbb{C},
$$

where the real function $H$ satisfies $H(T, \xi, \bar{\xi})=H(0, \xi, \bar{\xi})$ and has the Taylor expansion

$$
H(\tau, \xi, \bar{\xi})=\sum_{\substack{i, j=0 \\ 2 \leq i+j \leq 5}}^{5} \frac{1}{i ! j !} h_{i j}(\tau) \xi^{i} \bar{\xi}^{j}+O\left(|\xi|^{6}\right)
$$

with $h_{i j}(T)=h_{i j}(0)$ and $h_{i j}=\bar{h}_{j i}$ so that $h_{i i}$ is real, while $v$ and its conjugate $\bar{v}$ are defined as

$$
\left\{\begin{aligned}
\dot{v}(\tau)-A(\tau) v+i \omega v & =0, \tau \in[0, T], \\
v(T)-v(0) & =0 \\
\int_{0}^{T}\langle v, v\rangle d \tau-1 & =0
\end{aligned}\right.
$$

These functions exist due to Lemma 2 of [25].

If we assume that (2.1) restricted to $W^{c}(\Gamma)$ has the $\mathrm{CH}$ periodic normal form in Table 1, as in the previous cases, we can find the functions $h_{i j}(\tau)$ by solving appropriate BVPs.

First we introduce the two needed adjoint eigenfunctions. The first one, namely, $\varphi^{*}$, satisfies (3.16), and the second one, namely, $v^{*}$, satisfies

$$
\left\{\begin{aligned}
\dot{v}^{*}(\tau)+A^{\mathrm{T}}(\tau) v^{*}+i \omega v^{*} & =0, \tau \in[0, T] \\
v^{*}(T)-v^{*}(0) & =0 \\
\int_{0}^{T}\left\langle v^{*}, v\right\rangle d \tau-1 & =0
\end{aligned}\right.
$$

Note that in [30] the last term in the differential equation for $v^{*}$ has the wrong sign. This error could have led to wrong values of the cubic normal form coefficient at the torus bifurcation computed by early versions of MATCONT.

As usual, we substitute (3.24) into (2.1), use the CH normal form from Table 1, (3.25), as well as (2.2), and collect the corresponding terms in order to find the needed normal form coefficients. 
The $\xi$-independent and the linear terms give rise to the usual identities

$$
\dot{u}_{0}=F\left(u_{0}\right), \quad \dot{v}-A(\tau) v+i \omega v=0, \quad \dot{\bar{v}}-A(\tau) \bar{v}-i \omega \bar{v}=0 .
$$

Collecting the coefficients of the $\xi^{2}$ - or $\bar{\xi}^{2}$-terms leads to the equation

$$
\dot{h}_{20}-A(\tau) h_{20}+2 i \omega h_{20}=B(\tau ; v, v)
$$

or its complex-conjugate. This equation has a unique solution $h_{20}$ satisfying $h_{20}(T)=h_{20}(0)$, since due to the spectral assumptions $e^{2 i \omega T}$ is not a multiplier of the critical cycle. Thus, $h_{20}$ can be found by solving

$$
\left\{\begin{aligned}
\dot{h}_{20}-A(\tau) h_{20}+2 i \omega h_{20}-B(\tau ; v, v) & =0, \tau \in[0, T] \\
h_{20}(T)-h_{20}(0) & =0
\end{aligned}\right.
$$

By collecting the $\xi \bar{\xi}$-terms we obtain an equation for $h_{11}$, namely,

$$
\dot{h}_{11}-A(\tau) h_{11}=B(\tau ; v, \bar{v})-\alpha_{1} \dot{u}_{0},
$$

which is to be solved in the space of the functions satisfying $h_{11}(T)=h_{11}(0)$. In this space the operator $\frac{d}{d \tau}-A(\tau)$ has a range space with codim 1 . As before, the null-eigenfunction of the adjoint operator $-\frac{d}{d \tau}-A^{\mathrm{T}}(\tau)$ is $\varphi^{*}$, given by (3.16), and thus because of the Fredholm solvability condition, we can easily obtain the needed value for $\alpha_{1}$,

$$
\alpha_{1}=\int_{0}^{T}\left\langle\varphi^{*}, B(\tau ; v, \bar{v})\right\rangle d \tau
$$

With $\alpha_{1}$ defined in this way, let $h_{11}$ be the unique solution of the BVP

$$
\left\{\begin{aligned}
\dot{h}_{11}-A(\tau) h_{11}-B(\tau ; v, \bar{v})+\alpha_{1} \dot{u}_{0} & =0, \tau \in[0, T], \\
h_{11}(T)-h_{11}(0) & =0 \\
\int_{0}^{T}\left\langle\varphi^{*}, h_{11}\right\rangle d \tau & =0 .
\end{aligned}\right.
$$

The coefficient of the third order term in the $\mathrm{CH}$ normal form from Table 1 is purely imaginary since the first Lyapunov coefficient vanishes at a $\mathrm{CH}$ point. We are now ready to compute this coefficient. In fact, if we collect the $\xi^{2} \bar{\xi}$-terms, we obtain

$$
\dot{h}_{21}-A(\tau) h_{21}+i \omega h_{21}=C(\tau ; v, v, \bar{v})+2 B\left(\tau ; v, h_{11}\right)+B\left(\tau ; \bar{v}, h_{20}\right)-2 i c v-2 \alpha_{1} \dot{v},
$$

which is to be solved in the space of functions satisfying $h_{21}(T)=h_{21}(0)$. In this space the operator $\frac{d}{d \tau}-A(\tau)+i \omega$ is singular, since $e^{i \omega T}$ is a multiplier of the critical cycle. So we can impose the usual Fredholm solvability condition taking (3.27) into account:

$$
\int_{0}^{T}\left\langle v^{*}, C(\tau ; v, v, \bar{v})+2 B\left(\tau ; v, h_{11}\right)+B\left(\tau ; \bar{v}, h_{20}\right)-2 i c v-2 \alpha_{1} \dot{v}\right\rangle d \tau=0 .
$$

This allows us to find the value of the coefficient $c$ of the $\mathrm{CH}$ normal form from Table 1,

$$
c=-\frac{i}{2} \int_{0}^{T}\left\langle v^{*}, C(\tau ; v, v, \bar{v})+2 B\left(\tau ; v, h_{11}\right)+B\left(\tau ; \bar{v}, h_{20}\right)-2 \alpha_{1} A(\tau) v\right\rangle d \tau+\alpha_{1} \omega,
$$


and, with $c$ defined in this way, we can find $h_{21}$ as the unique solution of the BVP

$$
\left\{\begin{aligned}
\dot{h}_{21}-A(\tau) h_{21}+i \omega h_{21}-C(\tau ; v, v, \bar{v})-2 B\left(\tau ; v, h_{11}\right) & \\
-B\left(\tau ; \bar{v}, h_{20}\right)+2 i c v+2 \alpha_{1}(A(\tau) v-i \omega v) & =0, \tau \in[0, T], \\
h_{21}(T)-h_{21}(0) & =0 \\
\int_{0}^{T}\left\langle v^{*}, h_{21}\right\rangle d \tau & =0 .
\end{aligned}\right.
$$

Collecting the $\xi^{3}$-terms gives us an equation for $h_{30}$,

$$
\dot{h}_{30}-A(\tau) h_{30}+3 i \omega h_{30}=C(\tau ; v, v, v)+3 B\left(\tau ; v, h_{20}\right),
$$

which has a unique solution $h_{30}$ satisfying $h_{30}(T)=h_{30}(0)$, since $e^{3 i \omega T}$ is not a multiplier of the critical cycle by the spectral assumptions. Thus, $h_{30}$ is the unique solution of the BVP

$$
\left\{\begin{aligned}
\dot{h}_{30}-A(\tau) h_{30}+3 i \omega h_{30}-C(\tau ; v, v, v)-3 B\left(\tau ; v, h_{20}\right) & =0, \tau \in[0, T], \\
h_{30}(T)-h_{30}(0) & =0 .
\end{aligned}\right.
$$

By collecting the $\xi^{3} \bar{\xi}$-terms we obtain an equation for $h_{31}$,

$$
\begin{aligned}
\dot{h}_{31}-A(\tau) h_{31}+2 i \omega h_{31}= & D(\tau ; v, v, v, \bar{v})+3 C\left(\tau ; v, v, h_{11}\right)+3 C\left(\tau ; v, \bar{v}, h_{20}\right)+3 B\left(\tau ; h_{11}, h_{20}\right) \\
& +3 B\left(\tau ; v, h_{21}\right)+B\left(\tau ; \bar{v}, h_{30}\right)-6 i c h_{20}-3 \alpha_{1} \dot{h}_{20},
\end{aligned}
$$

which has a unique solution $h_{31}$ satisfying $h_{31}(T)=h_{31}(0)$, since $e^{2 i \omega T}$ is not a multiplier of the critical cycle by the spectral assumptions. Thus, $h_{31}$ is the unique solution of the BVP

$$
\left\{\begin{aligned}
\dot{h}_{31}-A(\tau) h_{31}+2 i \omega h_{31}-D(\tau ; v, v, v, \bar{v})-3 C\left(\tau ; v, v, h_{11}\right) & \\
-3 C\left(\tau ; v, \bar{v}, h_{20}\right)-3 B\left(\tau ; h_{11}, h_{20}\right)-3 B\left(\tau ; v, h_{21}\right) & \\
-B\left(\tau ; \bar{v}, h_{30}\right)+6 i c h_{20}+3 \alpha_{1}\left(A(\tau) h_{20}-2 i \omega h_{20}+B(\tau ; v, v)\right) & =0, \quad \tau \in[0, T], \\
h_{31}(T)-h_{31}(0) & =0 .
\end{aligned}\right.
$$

Taking the $|\xi|^{4}$-terms into account gives an equation for $h_{22}$,

$$
\begin{aligned}
\dot{h}_{22}-A(\tau) h_{22}= & D(\tau ; v, v, \bar{v}, \bar{v})+C\left(\tau ; v, v, h_{02}\right)+4 C\left(\tau ; v, \bar{v}, h_{11}\right)+C\left(\tau ; \bar{v}, \bar{v}, h_{20}\right) \\
& +2 B\left(\tau ; h_{11}, h_{11}\right)+2 B\left(\tau ; v, h_{12}\right)+B\left(\tau ; h_{02}, h_{20}\right)+2 B\left(\tau ; \bar{v}, h_{21}\right) \\
& -4 \alpha_{1} \dot{h}_{11}-4 \alpha_{2} \dot{u}_{0},
\end{aligned}
$$

which is to be solved in the space of functions satisfying $h_{22}(T)=h_{22}(0)$. In this space the operator $\frac{d}{d \tau}-A(\tau)$ has a range space with codim 1 which is orthogonal to $\varphi^{*}$. So one Fredholm solvability condition is involved, allowing us to compute the value of the coefficient $\alpha_{2}$ of our normal form as follows:

$$
\begin{aligned}
\alpha_{2}= & \frac{1}{4} \int_{0}^{T}\left\langle\varphi^{*}, D(\tau ; v, v, \bar{v}, \bar{v})+C\left(\tau ; v, v, h_{02}\right)+4 C\left(\tau ; v, \bar{v}, h_{11}\right)+C\left(\tau ; \bar{v}, \bar{v}, h_{20}\right)\right. \\
& +2 B\left(\tau ; h_{11}, h_{11}\right)+2 B\left(\tau ; v, h_{12}\right)+B\left(\tau ; h_{02}, h_{20}\right)+2 B\left(\tau ; \bar{v}, h_{21}\right) \\
& \left.-4 \alpha_{1}\left(A(\tau) h_{11}+B(\tau ; v, \bar{v})\right)\right\rangle d \tau+\alpha_{1}^{2} .
\end{aligned}
$$


Using this value for $\alpha_{2}$, we can find $h_{22}$ as the unique solution of the BVP

$$
\left\{\begin{aligned}
\dot{h}_{22}-A(\tau) h_{22}-D(\tau ; v, v, \bar{v}, \bar{v})-C\left(\tau ; v, v, h_{02}\right)-4 C\left(\tau ; v, \bar{v}, h_{11}\right) & \\
-C\left(\tau ; \bar{v}, \bar{v}, h_{20}\right)-2 B\left(\tau ; h_{11}, h_{11}\right)-2 B\left(\tau ; v, h_{12}\right)-B\left(\tau ; h_{02}, h_{20}\right) & \\
-2 B\left(\tau ; \bar{v}, h_{21}\right)+4 \alpha_{1}\left(A(\tau) h_{11}+B(\tau ; v, \bar{v})-\alpha_{1} F\left(u_{0}\right)\right)+4 \alpha_{2} F\left(u_{0}\right) & =0, \tau \in[0, T], \\
h_{22}(T)-h_{22}(0) & =0 \\
\int_{0}^{T}\left\langle\varphi^{*}, h_{22}\right\rangle d \tau & =0 .
\end{aligned}\right.
$$

Finally, by collecting the $\xi^{3} \bar{\xi}^{2}$-terms we get an equation for $h_{32}$,

$$
\begin{aligned}
& \dot{h}_{32}-A(\tau) h_{32}+i \omega h_{32}=E(\tau ; v, v, v, \bar{v}, \bar{v})+D\left(\tau ; v, v, v, h_{02}\right)+6 D\left(\tau ; v, v, \bar{v}, h_{11}\right) \\
& \quad+3 D\left(\tau ; v, \bar{v}, \bar{v}, h_{20}\right)+6 C\left(\tau ; v, h_{11}, h_{11}\right)+3 C\left(\tau ; v, v, h_{12}\right)+3 C\left(\tau ; v, h_{02}, h_{20}\right) \\
& \quad+6 C\left(\tau ; \bar{v}, h_{11}, h_{20}\right)+6 C\left(\tau ; v, \bar{v}, h_{21}\right)+C\left(\tau ; \bar{v}, \bar{v}, h_{30}\right)+3 B\left(\tau ; h_{12}, h_{20}\right)+6 B\left(\tau ; h_{11}, h_{21}\right) \\
& \quad+3 B\left(\tau ; v, h_{22}\right)+B\left(\tau ; h_{02}, h_{30}\right)+2 B\left(\tau ; \bar{v}, h_{31}\right)-12 e v-6 i c h_{21}-12 \alpha_{2} \dot{v}-6 \alpha_{1} \dot{h}_{21},
\end{aligned}
$$

which, since the operator is singular, allows us, using the first equation of (3.26) as well as the first and the last equations of (3.29), to compute the critical coefficient $e$ of the $\mathrm{CH}$ normal form by imposing the Fredholm solvability condition:

$$
\begin{aligned}
e=\frac{1}{12} \int_{0}^{T} & \left\langle v^{*}, E(\tau ; v, v, v, \bar{v}, \bar{v})+D\left(\tau ; v, v, v, h_{02}\right)+6 D\left(\tau ; v, v, \bar{v}, h_{11}\right)\right. \\
& +3 D\left(\tau ; v, \bar{v}, \bar{v}, h_{20}\right)+6 C\left(\tau ; v, h_{11}, h_{11}\right)+3 C\left(\tau ; v, v, h_{12}\right) \\
& +3 C\left(\tau ; v, h_{02}, h_{20}\right)+6 C\left(\tau ; \bar{v}, h_{11}, h_{20}\right)+6 C\left(\tau ; v, \bar{v}, h_{21}\right)+C\left(\tau ; \bar{v}, \bar{v}, h_{30}\right) \\
& +3 B\left(\tau ; h_{12}, h_{20}\right)+6 B\left(\tau ; h_{11}, h_{21}\right)+3 B\left(\tau ; v, h_{22}\right)+B\left(\tau ; h_{02}, h_{30}\right) \\
& +2 B\left(\tau ; \bar{v}, h_{31}\right)-12 \alpha_{2} A(\tau) v-6 \alpha_{1}\left(A(\tau) h_{21}+2 B\left(\tau ; v, h_{11}\right)+C(\tau ; v, v, \bar{v})\right. \\
& \left.\left.+B\left(\tau ; \bar{v}, h_{20}\right)-2 \alpha_{1} A v\right)\right\rangle d \tau+i \omega \alpha_{2}+i c \alpha_{1}-\alpha_{1}^{2} i \omega .
\end{aligned}
$$

We define the second Lyapunov coefficient as

$$
L_{2}(0)=\Re(e) \text {. }
$$

If this coefficient does not vanish, the codim 2 point is nondegenerate.

It can be checked that the equations for $h_{40}, h_{50}$, and $h_{41}$ are uniquely solvable. Since we are in a complex eigenvalue case, $v$ is determined up to a factor $\gamma$, for which $\bar{\gamma}^{\mathrm{T}} \gamma=1$. Then $v^{*}, h_{20}, h_{21}, h_{30}, h_{31}$ are replaced by $\gamma v^{*}, \gamma^{2} h_{20}, \gamma h_{21}, \gamma^{3} h_{30}, \gamma^{2} h_{31}$, respectively, but $\alpha_{1}, \alpha_{2}, c$, and $e$ are not affected by this factor.

3.2.2. Strong resonance $1: 1$ bifurcation. The three-dimensional critical center manifold $W^{c}(\Gamma)$ at the R1 bifurcation can be parameterized locally by $(\tau, \xi)$ as

$$
u=u_{0}(\tau)+\xi_{1} v_{1}(\tau)+\xi_{2} v_{2}(\tau)+H(\tau, \xi), \quad \tau \in[0, T], \xi=\left(\xi_{1}, \xi_{2}\right) \in \mathbb{R}^{2},
$$

where $H$ satisfies $H(T, \xi)=H(0, \xi)$ and has the Taylor expansion

$$
H(\tau, \xi)=\frac{1}{2} h_{20}(\tau) \xi_{1}^{2}+h_{11}(\tau) \xi_{1} \xi_{2}+\frac{1}{2} h_{02}(\tau) \xi_{2}^{2}+O\left(\|\xi\|^{3}\right) .
$$


Here the functions $h_{20}, h_{11}$, and $h_{02}$ are $T$-periodic in $\tau$, while $v_{1}$ and $v_{2}$ are the generalized eigenfunctions associated with the trivial multiplier and defined as the unique solutions of the BVPs

$$
\left\{\begin{aligned}
\dot{v}_{1}-A(\tau) v_{1}-F\left(u_{0}\right) & =0, \tau \in[0, T], \\
v_{1}(T)-v_{1}(0) & =0 \\
\int_{0}^{T}\left\langle v_{1}, F\left(u_{0}\right)\right\rangle d \tau & =0
\end{aligned}\right.
$$

and

$$
\left\{\begin{aligned}
\dot{v}_{2}-A(\tau) v_{2}+v_{1} & =0, \tau \in[0, T], \\
v_{2}(T)-v_{2}(0) & =0 \\
\int_{0}^{T}\left\langle v_{2}, F\left(u_{0}\right)\right\rangle d \tau & =0,
\end{aligned}\right.
$$

respectively. The functions $v_{1}$ and $v_{2}$ exist and are different due to Lemma 2 of [25]. Following our approach to find the value of the normal form constants, we define $\varphi^{*}$ as a solution of the adjoint eigenfunction problem (3.4), $v_{1}^{*}$ as a solution of

$$
\left\{\begin{aligned}
\dot{v}_{1}^{*}(\tau)+A^{\mathrm{T}}(\tau) v_{1}^{*}-\varphi^{*} & =0, \tau \in[0, T] \\
v_{1}^{*}(T)-v_{1}^{*}(0) & =0
\end{aligned}\right.
$$

and $v_{2}^{*}$ as a solution of

$$
\left\{\begin{aligned}
\dot{v}_{2}^{*}(\tau)+A^{\mathrm{T}}(\tau) v_{2}^{*}+v_{1}^{*} & =0, \tau \in[0, T] \\
v_{2}^{*}(T)-v_{2}^{*}(0) & =0
\end{aligned}\right.
$$

The above definitions immediately imply that

$$
\int_{0}^{T}\left\langle\varphi^{*}, F\left(u_{0}\right)\right\rangle d \tau=\int_{0}^{T}\left\langle\varphi^{*}, v_{1}\right\rangle d \tau=\int_{0}^{T}\left\langle F\left(u_{0}\right), v_{1}^{*}\right\rangle d \tau=0 .
$$

Due to the spectral assumptions at the $\mathrm{R} 1$ point we are free to assume that

$$
\int_{0}^{T}\left\langle\varphi^{*}, v_{2}\right\rangle d \tau=1
$$

Appending this condition to the eigenproblem, we can find the eigenfunction $\varphi^{*}$ as the unique solution of the BVP

$$
\left\{\begin{aligned}
\dot{\varphi}^{*}+A^{\mathrm{T}}(\tau) \varphi^{*} & =0, \tau \in[0, T], \\
\varphi^{*}(T)-\varphi^{*}(0) & =0 \\
\int_{0}^{T}\left\langle\varphi^{*}, v_{2}\right\rangle d \tau-1 & =0 .
\end{aligned}\right.
$$

As already mentioned in the CPC case, we will choose adjoint generalized eigenfunctions orthogonal to an original eigenfunction. Therefore, $v_{1}^{*}$ and $v_{2}^{*}$ are obtained as the solution of

$$
\left\{\begin{aligned}
\dot{v}_{1}^{*}+A^{\mathrm{T}}(\tau) v_{1}^{*}-\varphi^{*} & =0, \tau \in[0, T], \\
v_{1}^{*}(T)-v_{1}^{*}(0) & =0 \\
\int_{0}^{T}\left\langle v_{1}^{*}, v_{2}\right\rangle d \tau & =0
\end{aligned}\right.
$$


and

$$
\left\{\begin{aligned}
\dot{v}_{2}{ }^{*}(\tau)+A^{\mathrm{T}}(\tau) v_{2}^{*}+v_{1}^{*} & =0, \tau \in[0, T], \\
v_{2}^{*}(T)-v_{2}^{*}(0) & =0 \\
\int_{0}^{T}\left\langle v_{2}^{*}, v_{2}\right\rangle d \tau & =0
\end{aligned}\right.
$$

respectively. Notice that, as in the CPC case, we have normalized in (3.35) the adjoint eigenfunction with the last generalized eigenfunction, which gives us in addition

$$
\int_{0}^{T}\left\langle v_{1}^{*}, v_{1}\right\rangle d \tau=\int_{0}^{T}\left\langle v_{2}^{*}, F\left(u_{0}\right)\right\rangle d \tau=1 .
$$

As usual, to derive the value of the normal form coefficients we substitute (3.30) into (2.1), we use (2.2) as well as the R1 normal form in Table 1 and (3.31), and we get differential equations at every degree of $\xi$. Note that in fact the solvability of all the equations up to the maximal order of the normal form has to be checked. We will pay extra attention to it in this section.

By collecting the $\xi^{0}$-terms we get the identity $\dot{u}_{0}=F\left(u_{0}\right)$. The linear terms provide two other identities, namely,

$$
\dot{v}_{1}-A(\tau) v_{1}-F\left(u_{0}\right)=0 \quad \text { and } \quad \dot{v}_{2}-A v_{2}+v_{1}=0 ;
$$

cf. (3.32) and (3.33).

By collecting the $\xi_{1}^{2}$-terms we find an equation for $h_{20}$, namely,

$$
\dot{h}_{20}-A(\tau) h_{20}=-2 \alpha \dot{u}_{0}+2 \dot{v}_{1}+B\left(\tau ; v_{1}, v_{1}\right)-2 a v_{2},
$$

which is to be solved in the space of periodic functions on $[0, T]$. In this space, the differential operator $\frac{d}{d \tau}-A(\tau)$ is singular with a range orthogonal to $\varphi^{*}$. Using (3.34), (3.35), and (3.32), we obtain from the corresponding Fredholm solvability condition the following value for $a$ :

$$
a=\frac{1}{2} \int_{0}^{T}\left\langle\varphi^{*}, 2 A(\tau) v_{1}+B\left(\tau ; v_{1}, v_{1}\right)\right\rangle d \tau
$$

Notice that in the RHS of (3.39) we have no freedom which could change the value of the coefficient $a$. This confirms the theoretically proved fact that the $\xi_{1}^{2}$-term of the R1 normal form from Table 1 is resonant. Notice, moreover, that parameter $\alpha$ is undetermined, which gives us two degrees of freedom for $h_{20}$. In fact, if $h_{20}$ is a solution of (3.39), then also $\tilde{h}_{20}=h_{20}+\varepsilon_{20}^{I} F\left(u_{0}\right)+\varepsilon_{20}^{I I} v_{1}$ is a solution, due to the fact that $F\left(u_{0}\right)$ spans the null-space of the operator $\frac{d}{d \tau}-A(\tau)$ and that we can tune $\alpha$ as desired:

$$
\frac{d \tilde{h}_{20}}{d \tau}-A(\tau) \tilde{h}_{20}=\frac{d h_{20}}{d \tau}-A(\tau) h_{20}+\varepsilon_{20}^{I I}\left(\frac{d v_{1}}{d \tau}-A(\tau) v_{1}\right)=\frac{d h_{20}}{d \tau}-A(\tau) h_{20}+\varepsilon_{20}^{I I} \dot{u}_{0}
$$

By collecting the $\xi_{1} \xi_{2}$-terms we find an equation for $h_{11}$,

$$
\dot{h}_{11}-A(\tau) h_{11}=B\left(\tau ; v_{1}, v_{2}\right)+\dot{v}_{2}-h_{20}-b v_{2}+v_{1},
$$


which is to be solved in the space of $T$-periodic functions. As in the previous case, taking (3.35) into account, as well as (3.33) and (3.34), the corresponding solvability condition implies

$$
b=\int_{0}^{T}\left\langle\varphi^{*}, B\left(\tau ; v_{1}, v_{2}\right)+A(\tau) v_{2}\right\rangle d \tau-\int_{0}^{T}\left\langle\varphi^{*}, h_{20}\right\rangle d \tau
$$

Using (3.37), (3.39), (3.34), and (3.32), we can rewrite this expression as

$$
b=\int_{0}^{T}\left\langle\varphi^{*}, B\left(\tau ; v_{1}, v_{2}\right)+A(\tau) v_{2}\right\rangle d \tau+\int_{0}^{T}\left\langle v_{1}^{*}, 2 A v_{1}+B\left(\tau ; v_{1}, v_{1}\right)\right\rangle d \tau,
$$

thus obtaining a formula for $b$ which involves only the original and adjoint eigenfunctions.

Notice that the freedom that we have in $h_{20}$ cannot be used to change the value of coefficient $b$ (and so the $\xi_{1} \xi_{2}$-term of the $\mathrm{R} 1$ normal form in Table 1 is resonant). Indeed, $h_{20}$ is defined up to a multiple of $F\left(u_{0}\right)$ and $v_{1}$, but both vectors are orthogonal to $\varphi^{*}$; see the first two orthogonality conditions in (3.34). However the presence of $h_{20}$ in the RHS gives us three degrees of freedom for $h_{11}$. In fact, if $h_{11}$ is a solution of (3.42), $\tilde{h}_{11}=h_{11}+\varepsilon_{11}^{I} F\left(u_{0}\right)-\varepsilon_{20}^{I} v_{1}+\varepsilon_{20}^{I I} v_{2}$ is also a solution.

Collecting the $\xi_{2}^{2}$-terms gives us an equation for $h_{02}$,

$$
\dot{h}_{02}-A(\tau) h_{02}=B\left(\tau, v_{2}, v_{2}\right)-2 h_{11}
$$

which is to be solved in the space of $T$-periodic functions. This equation should be solvable, so the RHS should lie in the range of the operator $\frac{d}{d t}-A(\tau)$ :

$$
\int_{0}^{T}\left\langle\varphi^{*}, B\left(\tau, v_{2}, v_{2}\right)-2 h_{11}\right\rangle d \tau=0
$$

This condition can be satisfied by tuning $h_{11}$. In fact, $\varepsilon_{20}^{I I}$ is not yet determined, so $h_{11}$ can have a projection on $v_{2}$. Due to (3.35), $v_{2}$ does not lie in the range of the $\frac{d}{d \tau}-A(\tau)$ operator, and therefore we can require that

$$
\int_{0}^{T}\left\langle\varphi^{*}, h_{11}\right\rangle d \tau=\frac{1}{2} \int_{0}^{T}\left\langle\varphi^{*}, B\left(\tau, v_{2}, v_{2}\right)\right\rangle d \tau
$$

This last solvability condition determines $\varepsilon_{20}^{I I}$ uniquely, and since $\varepsilon_{20}^{I I}$ determines the value of $\alpha$ (see (3.39) and (3.41)), $\alpha$ is now also uniquely determined. So the center manifold expansion has now become unique. Note that in fact the value of $\alpha$ is not needed since it can be shown that it does not affect the bifurcation scenario. Note also that in order to compute the necessary coefficients $a$ and $b$ by (3.40) and (3.43), the second order expansion of the center manifold is not needed. Indeed, we have rewritten the formulas of the normal form coefficients in terms of the original and adjoint eigenfunctions. Since $h_{20}$ and $h_{11}$ are not needed, we do not write down their defining BVPs. 
3.2.3. Strong resonance 1:2 bifurcation. The three-dimensional critical center manifold $W^{c}(\Gamma)$ at the $\mathrm{R} 2$ bifurcation can be parameterized locally by $(\tau, \xi)$ as

$$
u=u_{0}(\tau)+\xi_{1} v_{1}(\tau)+\xi_{2} v_{2}(\tau)+H(\tau, \xi), \quad \tau \in[0,2 T], \xi=\left(\xi_{1}, \xi_{2}\right) \in \mathbb{R}^{2},
$$

where $H$ satisfies $H(2 T, \xi)=H(0, \xi)$ and has the Taylor expansion

$$
H(\tau, \xi)=\sum_{\substack{i, j=0 \\ 2 \leq i+j \leq 3}}^{3} \frac{1}{i ! j !} h_{i j}(\tau) \xi_{1}^{i} \xi_{2}^{j}+O\left(\|\xi\|^{4}\right),
$$

where all functions $h_{i j}$ are $2 T$-periodic, the eigenfunction corresponding to eigenvalue -1 is given by

$$
\left\{\begin{aligned}
\dot{v}_{1}-A(\tau) v_{1} & =0, \tau \in[0, T], \\
v_{1}(T)+v_{1}(0) & =0 \\
\int_{0}^{T}\left\langle v_{1}, v_{1}\right\rangle d \tau-1 & =0
\end{aligned}\right.
$$

and the generalized eigenfunction is given by

$$
\left\{\begin{aligned}
\dot{v}_{2}-A(\tau) v_{2}+v_{1} & =0, \tau \in[0, T], \\
v_{2}(T)+v_{2}(0) & =0 \\
\int_{0}^{T}\left\langle v_{2}, v_{1}\right\rangle d \tau & =0
\end{aligned}\right.
$$

with

$$
v_{1}(\tau+T):=-v_{1}(\tau) \text { and } v_{2}(\tau+T):=-v_{2}(\tau) \text { for } \tau \in[0, T] .
$$

The functions $v_{1}$ and $v_{2}$ exist due to Lemma 5 of [25]. The functions $h_{i j}$ of (3.45) can be found by solving appropriate BVPs, assuming that (2.1) restricted to $W^{c}(\Gamma)$ has the R2 normal form from Table 1. As in the GPD case, we first deduce periodicity properties of these functions $h_{i j}$. Similarly to the GPD case, we here have $u\left(\tau, \xi_{1}, \xi_{2}\right)=u\left(\tau+T,-\xi_{1},-\xi_{2}\right)$. This implies that

$$
\sum_{i, j} \frac{1}{i ! j !} h_{i j}(\tau) \xi_{1}^{i} \xi_{2}^{j}=\sum_{i, j} \frac{1}{i ! j !} h_{i j}(\tau+T)(-1)^{i+j} \xi_{1}^{i} \xi_{2}^{j},
$$

and thus

$$
h_{i j}(\tau)=(-1)^{i+j} h_{i j}(\tau+T),
$$

from which follows that $h_{i j}(\tau+T)=h_{i j}(\tau)$ for $i+j$ even and $h_{i j}(\tau+T)=-h_{i j}(\tau)$ for $i+j$ odd, for $\tau \in[0, T]$. Taking these (anti)periodicity properties into account, we can reduce our analysis to the interval $[0, T]$ instead of $[0,2 T]$.

The coefficients $\alpha, a$, and $b$ arise from the solvability conditions for the BVPs as integrals of scalar products over the interval $[0, T]$. Specifically, those scalar products involve among other things the quadratic and cubic terms of (2.2) near the periodic solution $u_{0}$. The adjoint eigenfunction $\varphi^{*}$ associated to the trivial multiplier is the $T$-periodic solution of (3.16). The adjoint eigenfunction $v_{1}^{*}$ is the unique solution of the problem

$$
\left\{\begin{aligned}
\dot{v}_{1}^{*}(\tau)+A^{\mathrm{T}}(\tau) v_{1}^{*} & =0, \tau \in[0, T], \\
v_{1}^{*}(T)+v_{1}^{*}(0) & =0 \\
\int_{0}^{T}\left\langle v_{1}^{*}, v_{2}\right\rangle d \tau-1 & =0 .
\end{aligned}\right.
$$


Note that we can indeed require this normalization since $v_{2}$ is the last generalized eigenfunction of the original problem and therefore not orthogonal to all the eigenfunctions of the adjoint problem. We further define the generalized adjoint eigenfunction $v_{2}^{*}$ as the unique solution of

$$
\left\{\begin{aligned}
\dot{v}_{2}^{*}(\tau)+A^{\mathrm{T}}(\tau) v_{2}^{*}-v_{1}^{*} & =0, \quad \tau \in[0, T], \\
v_{2}^{*}(T)+v_{2}^{*}(0) & =0 \\
\int_{0}^{T}\left\langle v_{2}^{*}, v_{2}\right\rangle d \tau & =0
\end{aligned}\right.
$$

since, as above, $v_{1}^{*}$ is not orthogonal to $v_{2}$. Moreover, we have

$$
\int_{0}^{T}\left\langle v_{2}^{*}, v_{1}\right\rangle d \tau=\int_{0}^{T}\left\langle v_{1}^{*}, v_{2}\right\rangle d \tau=1
$$

and

$$
\int_{0}^{T}\left\langle v_{2}, v_{1}\right\rangle d \tau=\int_{0}^{T}\left\langle v_{1}^{*}, v_{1}\right\rangle d \tau=0 .
$$

To derive the normal form coefficients, we proceed as in the previous sections, namely, we substitute (3.44) into (2.1), and use (2.2) as well as the R2 normal form from Table 1 and (3.45).

By collecting the $\xi^{0}$-terms we get the trivial identity $\dot{u}_{0}=F\left(u_{0}\right)$. The linear terms provide two other identities, namely, $\dot{v}_{1}=A(\tau) v_{1}$ and $v_{1}+\dot{v}_{2}=A(\tau) v_{2}$, in correspondence with (3.46) and (3.47).

Collecting the $\xi_{2}^{2}$-terms gives an equation for $h_{02}$,

$$
\dot{h}_{02}-A(\tau) h_{02}=B\left(\tau ; v_{2}, v_{2}\right)-2 h_{11}
$$

which is to be solved in the space of functions satisfying $h_{02}(T)=h_{02}(0)$. In this space, the differential operator $\frac{d}{d \tau}-A(\tau)$ is singular and its null-space is spanned by $\dot{u}_{0}$. The Fredholm solvability condition gives a normalization condition for function $h_{11}$, namely,

$$
\int_{0}^{T}\left\langle\varphi^{*}, h_{11}\right\rangle d \tau=\frac{1}{2} \int_{0}^{T}\left\langle\varphi^{*}, B\left(\tau ; v_{2}, v_{2}\right)\right\rangle d \tau
$$

By collecting the $\xi_{1} \xi_{2}$-terms we obtain the differential equation for $h_{11}$,

$$
\dot{h}_{11}-A(\tau) h_{11}=B\left(\tau ; v_{1}, v_{2}\right)-h_{20},
$$

which must be solved in the space of functions satisfying $h_{11}(T)=h_{11}(0)$. The Fredholm solvability condition gives in this case a normalization condition for $h_{20}$, i.e.,

$$
\int_{0}^{T}\left\langle\varphi^{*}, h_{20}\right\rangle d \tau=\int_{0}^{T}\left\langle\varphi^{*}, B\left(\tau ; v_{1}, v_{2}\right)\right\rangle d \tau
$$

By collecting the $\xi_{1}^{2}$-terms we find an equation for $h_{20}$,

$$
\dot{h}_{20}-A(\tau) h_{20}=B\left(\tau ; v_{1}, v_{1}\right)-2 \alpha \dot{u}_{0},
$$


which is to be solved in the space of functions satisfying $h_{20}(T)=h_{20}(0)$. In this space, the differential operator $\frac{d}{d \tau}-A(\tau)$ is singular and its null-space is spanned by $\dot{u}_{0}$. The Fredholm solvability condition leads to the expression

$$
\alpha=\frac{1}{2} \int_{0}^{T}\left\langle\varphi^{*}, B\left(\tau ; v_{1}, v_{1}\right)\right\rangle d \tau
$$

where $v_{1}$ is defined in (3.46).

With $\alpha$ defined in this way we have to find a normalization condition which makes the solution of (3.52) unique. Indeed, if $h_{20}$ is a solution of (3.52) with $h_{20}(T)=h_{20}(0)$, then $\tilde{h}_{20}=h_{20}+\varepsilon_{1} \dot{u}_{0}$ is also a solution, since $\dot{u}_{0}$ spans the kernel of the operator $\frac{d}{d \tau}-A(\tau)$ in the space of $T$-periodic functions. The projection along the space generated by $\dot{u}_{0}$ is fixed by solvability condition (3.51). So $h_{20}$ can be found as the unique solution of the BVP

$$
\left\{\begin{aligned}
\dot{h}_{20}-A(\tau) h_{20}-B\left(\tau ; v_{1}, v_{1}\right)+2 \alpha F\left(u_{0}\right) & =0, \tau \in[0, T], \\
h_{20}(T)-h_{20}(0) & =0, \\
\int_{0}^{T}\left\langle\varphi^{*}, h_{20}\right\rangle d \tau & =\int_{0}^{T}\left\langle\varphi^{*}, B\left(\tau ; v_{1}, v_{2}\right)\right\rangle d \tau .
\end{aligned}\right.
$$

In line with the previous observations, we can define $h_{11}$ as the unique solution of the BVP

$$
\left\{\begin{aligned}
\dot{h}_{11}-A(\tau) h_{11}-B\left(\tau ; v_{1}, v_{2}\right)+h_{20} & =0, \tau \in[0, T] \\
h_{11}(T)-h_{11}(0) & =0 \\
\int_{0}^{T}\left\langle\varphi^{*}, h_{11}\right\rangle d \tau & =\frac{1}{2} \int_{0}^{T}\left\langle\varphi^{*}, B\left(\tau ; v_{2}, v_{2}\right)\right\rangle d \tau
\end{aligned}\right.
$$

with $h_{20}$ defined in (3.54).

By collecting the $\xi_{1}^{3}$-terms we get an equation for $h_{30}$,

$$
\dot{h}_{30}-A(\tau) h_{30}=C\left(\tau ; v_{1}, v_{1}, v_{1}\right)+3 B\left(\tau ; v_{1}, h_{20}\right)-6 a v_{2}-6 \alpha \dot{v}_{1},
$$

which again must be solved in the space of functions satisfying $h_{30}(T)=-h_{30}(0)$. Taking the integral condition of (3.48) into account, we obtain

$$
a=\frac{1}{6} \int_{0}^{T}\left\langle v_{1}^{*}, C\left(\tau ; v_{1}, v_{1}, v_{1}\right)+3 B\left(\tau ; v_{1}, h_{20}\right)-6 \alpha A(\tau) v_{1}\right\rangle d \tau,
$$

where $\alpha$ is defined by (3.53), $h_{20}$ is the solution of (3.54), and $v_{1}$ and $v_{1}^{*}$ are defined in (3.46) and (3.47), respectively. As remarked before, it is important to note that if $h_{30}$ is a solution of (3.55) with $h_{30}(T)=-h_{30}(0)$, then $\tilde{h}_{30}=h_{30}+\epsilon_{30}^{I} v_{1}$ is also a solution, since $v_{1}$ spans the null-space of the operator $\frac{d}{d t}-A(\tau)$.

Collecting the $\xi_{1}^{2} \xi_{2}$-terms we get the equation for $h_{21}$,

$\dot{h}_{21}-A(\tau) h_{21}=-h_{30}-2 b v_{2}-2 \alpha \dot{v}_{2}-2 \alpha v_{1}+C\left(\tau ; v_{1}, v_{1}, v_{2}\right)+B\left(\tau ; h_{20}, v_{2}\right)+2 B\left(\tau ; h_{11}, v_{1}\right)$,

which is to be solved in the space of functions satisfying $h_{21}(T)=-h_{21}(0)$. The solvability of this equation implies that

$$
\int_{0}^{T}\left\langle v_{1}^{*},-h_{30}-2 b v_{2}-2 \alpha \dot{v}_{2}-2 \alpha v_{1}+C\left(\tau ; v_{1}, v_{1}, v_{2}\right)+B\left(\tau ; h_{20}, v_{2}\right)+2 B\left(\tau ; h_{11}, v_{1}\right)\right\rangle d \tau=0
$$


Notice that the $\xi_{1}^{2} \xi_{2}$-term in the R2 normal form from Table 1 is resonant: in fact, we cannot use the freedom on $h_{30}$ to make the normal form parameter $b$ zero since

$$
\int_{0}^{T}\left\langle v_{1}^{*}, \tilde{h}_{30}\right\rangle d \tau=\int_{0}^{T}\left\langle v_{1}^{*}, h_{30}+\varepsilon_{30}^{I} v_{1}\right\rangle d \tau=\int_{0}^{T}\left\langle v_{1}^{*}, h_{30}\right\rangle d \tau .
$$

Using the normalization from (3.48) and (3.50) gives us the following expression for $b$ :

$b=\frac{1}{2} \int_{0}^{T}\left\langle v_{1}^{*},-2 \alpha A(\tau) v_{2}+C\left(\tau ; v_{1}, v_{1}, v_{2}\right)+B\left(\tau ; h_{20}, v_{2}\right)+2 B\left(\tau ; h_{11}, v_{1}\right)\right\rangle d \tau-\frac{1}{2} \int_{0}^{T}\left\langle v_{1}^{*}, h_{30}\right\rangle d \tau$

However, there is no need to explicitly compute the cubic expansion of the center manifold since the last term of this sum can be rewritten so that the formula for $b$ takes the form

$$
\begin{gathered}
b=\frac{1}{2} \int_{0}^{T}\left\langle v_{1}^{*},-2 \alpha A(\tau) v_{2}+C\left(\tau ; v_{1}, v_{1}, v_{2}\right)+B\left(\tau ; h_{20}, v_{2}\right)+2 B\left(\tau ; h_{11}, v_{1}\right)\right\rangle d \tau \\
+\frac{1}{2} \int_{0}^{T}\left\langle v_{2}^{*}, C\left(\tau ; v_{1}, v_{1}, v_{1}\right)+3 B\left(\tau ; v_{1}, h_{20}\right)-6 \alpha A v_{1}\right\rangle d \tau
\end{gathered}
$$

where $h_{20}$ is defined in (3.54) and $\alpha$ calculated in (3.53). Notice that, since $h_{30}$ appears on the RHS of (3.56), we have two degrees of freedom on $h_{21}$. In fact, if $h_{21}$ is a solution of (3.56), then $\tilde{h}_{21}=h_{21}+\varepsilon_{21}^{I} v_{1}+\varepsilon_{30}^{I} v_{2}$ is also a solution since

$$
\frac{d \tilde{h}_{21}}{d \tau}-A(\tau) \tilde{h}_{21}=\frac{d h_{21}}{d \tau}-A(\tau) h_{21}+\varepsilon_{30}^{I}\left(\frac{d v_{2}}{d \tau}-A(\tau) v_{2}\right)=\frac{d h_{21}}{d \tau}-A(\tau) h_{21}-\varepsilon_{30}^{I} v_{1}
$$

By collecting the $\xi_{1} \xi_{2}^{2}$-terms we get the equation for $h_{12}$,

$$
\dot{h}_{12}-A(\tau) h_{12}=C\left(\tau, v_{1}, v_{2}, v_{2}\right)+B\left(\tau, v_{1}, h_{02}\right)+2 B\left(\tau, v_{2}, h_{11}\right)-2 h_{21},
$$

which is to be solved in the space of functions satisfying $h_{12}(T)=-h_{12}(0)$. The Fredholm solvability condition implies that

$$
\int_{0}^{T}\left\langle v_{1}^{*}, C\left(\tau, v_{1}, v_{2}, v_{2}\right)+B\left(\tau, v_{1}, h_{02}\right)+2 B\left(\tau, v_{2}, h_{11}\right)-2 h_{21}\right\rangle d \tau=0 .
$$

As mentioned before, $h_{21}$ has a component in the direction of $v_{2}$, which is not orthogonal to the adjoint eigenfunction $v_{1}^{*}$, so it is possible to impose

$$
\int_{0}^{T}\left\langle v_{1}^{*}, h_{21}\right\rangle d \tau=\frac{1}{2} \int_{0}^{T}\left\langle v_{1}^{*}, C\left(\tau, v_{1}, v_{2}, v_{2}\right)+B\left(\tau, v_{1}, h_{02}\right)+2 B\left(\tau, v_{2}, h_{11}\right)\right\rangle d \tau .
$$

This condition defines $\varepsilon_{30}^{I}$ uniquely; the freedom of $\varepsilon_{21}^{I}$ gives us as usual another freedom on $h_{12}$ in the direction of $v_{2}$.

Finally, collecting the $\xi_{2}^{3}$-terms gives

$$
\dot{h}_{03}-A(\tau) h_{03}=C\left(\tau, v_{2}, v_{2}, v_{2}\right)+3 B\left(v_{2}, h_{02}\right)-3 h_{12},
$$


which is to be solved in the space of functions satisfying $h_{03}(T)=-h_{03}(0)$. The Fredholm solvability condition is

$$
\int_{0}^{T}\left\langle v_{1}^{*}, C\left(\tau, v_{2}, v_{2}, v_{2}\right)+3 B\left(v_{2}, h_{02}\right)-3 h_{12}\right\rangle d \tau=0,
$$

which can be satisfied imposing

$$
\int_{0}^{T}\left\langle v_{1}^{*}, h_{12}\right\rangle d \tau=\frac{1}{3} \int_{0}^{T}\left\langle v_{1}^{*}, C\left(\tau, v_{2}, v_{2}, v_{2}\right)+3 B\left(v_{2}, h_{02}\right)\right\rangle d \tau .
$$

This last condition determines the value of $\varepsilon_{21}^{I}$, and thus the third order center manifold expansion is uniquely determined. However, since this third order expansion of the center manifold is not needed for the computation of the critical coefficients, we do not write down those conditions explicitly.

3.2.4. Strong resonance 1:3 bifurcation. The three-dimensional critical center manifold $W^{c}(\Gamma)$ at the R3 bifurcation can be parameterized locally by $(\tau, \xi)$ as

$$
u=u_{0}(\tau)+\xi v(\tau)+\bar{\xi} \bar{v}(\tau)+H(\tau, \xi, \bar{\xi}), \quad \tau \in[0,3 T], \quad \xi \in \mathbb{C},
$$

where the real function $H$ satisfies $H(3 T, \xi, \bar{\xi})=H(0, \xi, \bar{\xi})$ and has the Taylor expansion

$$
H(\tau, \xi, \bar{\xi})=\sum_{\substack{i, j=0 \\ 2 \leq i+j \leq 3}}^{3} \frac{1}{i ! j !} h_{i j}(\tau) \xi^{i} \bar{\xi}^{j}+O\left(|\xi|^{4}\right)
$$

with $h_{i j}(3 T)=h_{i j}(0)$ and $h_{i j}=\bar{h}_{j i}$ so that $h_{i i}$ is real. The eigenfunction $v$ is defined as the unique solution of the BVP

$$
\left\{\begin{aligned}
\dot{v}(\tau)-A(\tau) v & =0, \tau \in[0, T] \\
v(T)-e^{i \frac{2 \pi}{3}} v(0) & =0 \\
\int_{0}^{T}\langle v, v\rangle d \tau-1 & =0
\end{aligned}\right.
$$

and is extended on the interval $[0,3 T]$ using the equivariance property of the normal form, i.e.,

$$
v(\tau+T):=e^{i \frac{2 \pi}{3}} v(\tau) \text { and } v(\tau+2 T):=e^{i \frac{4 \pi}{3}} v(\tau) \text { for } \tau \in[0, T] .
$$

The definition of the conjugate eigenfunction $\bar{v}$ follows immediately. These functions exist due to Lemma 2 of [25].

As usual the functions $h_{i j}$ can be found by solving appropriate BVPs, assuming that (2.1) restricted to $W^{c}(\Gamma)$ has the periodic R3 normal form from Table 1. Also here we can deduce a property for the functions $h_{i j}$. The definition of $v(\tau)$ in $[0,3 T]$ states that $u(\tau, \xi, \bar{\xi})=u\left(\tau+T, e^{-i 2 \pi / 3} \xi, e^{i 2 \pi / 3} \bar{\xi}\right)$. Therefore,

$$
\sum_{k, l} \frac{1}{k ! l !} h_{k l}(\tau) \xi^{k} \bar{\xi}^{l}=\sum_{k, l} \frac{1}{k ! l !} h_{k l}(\tau+T)\left(e^{-i 2 \pi / 3}\right)^{k} \xi^{k}\left(e^{i 2 \pi / 3}\right)^{l} \bar{\xi}^{l},
$$


and thus

$$
h_{k l}(\tau)=h_{k l}(\tau+T)\left(e^{-i 2 \pi / 3}\right)^{k}\left(e^{i 2 \pi / 3}\right)^{l}
$$

for $\tau \in[0, T]$. This, for example, implies that $h_{k k}$ is $T$-periodic. These periodicity properties allow us to concentrate only on the interval $[0, T]$.

The adjoint eigenfunction $\varphi^{*}$ corresponding to the trivial multiplier is the unique $T$ periodic solution of BVP (3.16). The adjoint eigenfunction $v^{*}$ satisfies

$$
\left\{\begin{aligned}
\dot{v}^{*}(\tau)+A^{\mathrm{T}}(\tau) v^{*} & =0, \tau \in[0, T] \\
v^{*}(T)-e^{i \frac{2 \pi}{3}} v^{*}(0) & =0 \\
\int_{0}^{T}\left\langle v^{*}, v\right\rangle d \tau-1 & =0
\end{aligned}\right.
$$

Similarly, we obtain $\bar{v}^{*}$.

After the standard substitutions in (2.1), the constant and linear terms give us as usual

$$
\dot{u}_{0}=F\left(u_{0}\right), \quad \dot{v}-A(\tau) v=0, \quad \dot{\bar{v}}-A(\tau) \bar{v}=0 .
$$

From the $\xi^{2}$ - or $\bar{\xi}^{2}$-terms we obtain the equation (or its complex-conjugate)

$$
\dot{h}_{20}-A(\tau) h_{20}=B(\tau ; v, v)-2 \bar{b} \bar{v}
$$

which is to be solved in the space of functions satisfying $h_{20}(T)=e^{i \frac{4 \pi}{3}} h_{20}(0)$. In this space the operator $\frac{d}{d \tau}-A(\tau)$ has a range space with codim 1 which is orthogonal to $\bar{v}^{*}$. So only one Fredholm solvability condition is involved, from which we obtain

$$
b=\frac{1}{2} \int_{0}^{T}\left\langle v^{*}, B(\tau ; \bar{v}, \bar{v})\right\rangle d \tau .
$$

Using this value for $b$, we can find $h_{20}$ as the unique solution of the BVP

$$
\left\{\begin{aligned}
\dot{h}_{20}-A(\tau) h_{20}-B(\tau ; v, v)+2 \bar{b} \bar{v} & =0, \quad \tau \in[0, T], \\
h_{20}(T)-e^{i \frac{4 \pi}{3}} h_{20}(0) & =0 \\
\int_{0}^{T}\left\langle\bar{v}^{*}, h_{20}\right\rangle d \tau & =0 .
\end{aligned}\right.
$$

By collecting the $\xi \bar{\xi}$-terms, we obtain an equation for $h_{11}$,

$$
\dot{h}_{11}-A(\tau) h_{11}=B(\tau ; v, \bar{v})-\alpha_{1} \dot{u}_{0},
$$

which is to be solved in the space of functions satisfying $h_{11}(T)=h_{11}(0)$. The Fredholm solvability condition implies

$$
\alpha_{1}=\int_{0}^{T}\left\langle\varphi^{*}, B(\tau ; v, \bar{v})\right\rangle d \tau
$$

With $\alpha_{1}$ defined in this way, let $h_{11}$ be the unique solution of the BVP

$$
\left\{\begin{aligned}
\dot{h}_{11}-A(\tau) h_{11}-B(\tau ; v, \bar{v})+\alpha_{1} \dot{u}_{0} & =0, \tau \in[0, T], \\
h_{11}(T)-h_{11}(0) & =0 \\
\int_{0}^{T}\left\langle\varphi^{*}, h_{11}\right\rangle d \tau & =0
\end{aligned}\right.
$$


Finally, collecting the $\xi^{2} \bar{\xi}$-terms gives an equation for $h_{21}$,

$$
\dot{h}_{21}-A(\tau) h_{21}=C(\tau ; v, v, \bar{v})+2 B\left(\tau ; v, h_{11}\right)+B\left(\tau ; \bar{v}, h_{20}\right)-2 c v-2 \bar{b} h_{02}-2 \alpha_{1} \dot{v},
$$

which is to be solved in the space of the functions satisfying $h_{21}(T)=e^{i \frac{2 \pi}{3}} h_{21}(0)$. The Fredholm solvability condition implies that parameter $c$ of the R3 normal form in Table 1 is determined by

$$
c=\frac{1}{2} \int_{0}^{T}\left\langle v^{*}, C(\tau ; v, v, \bar{v})+2 B\left(\tau ; v, h_{11}\right)+B\left(\tau ; \bar{v}, h_{20}\right)-2 \alpha_{1} A v\right\rangle d \tau,
$$

where $\alpha_{1}$ is defined by (3.61), and $v, h_{11}$, and $h_{20}$ are the unique solutions of the BVPs (3.57), (3.62), and (3.60).

By collecting the $\xi^{3}$-terms we obtain

$$
\dot{h}_{30}-A(\tau) h_{30}=C(\tau ; v, v, v)+3 B\left(\tau ; v, h_{20}\right)-6 \bar{b} h_{11}-6 \alpha_{2} \dot{u}_{0},
$$

which is to be solved in the space of the functions satisfying $h_{30}(T)=h_{30}(0)$. Therefore,

$$
\alpha_{2}=\int_{0}^{T}\left\langle\varphi^{*}, C(\tau ; v, v, v)+3 B\left(\tau ; v, h_{20}\right)\right\rangle d \tau
$$

Note that as in the $\mathrm{CH}$ case $v$ is not uniquely determined. Indeed, when $v$ is a solution of (3.57) and $\gamma \in \mathbb{C}$ with $\bar{\gamma} \gamma=1$, then $\gamma v$ is also a solution. Then the adjoint function is given by $\gamma v^{*}$, and $b$ and $h_{20}$ are replaced by $\bar{\gamma}^{3} b$ and $\gamma^{2} h_{20}$, respectively. The normal form coefficient $c$ remains the same. However, the normal form coefficient $b$ is multiplied by $\bar{\gamma}^{3}$. This does not affect the bifurcation analysis since it must hold only that this normal form coefficient is nonzero, and obviously $\gamma \neq 0$. Moreover, the analysis around the bifurcation point is independent from the sign of $b$.

3.2.5. Strong resonance 1:4 bifurcation. The three-dimensional critical center manifold $W^{c}(\Gamma)$ at the R4 bifurcation can be parameterized locally by $(\tau, \xi)$ as

$$
u=u_{0}(\tau)+\xi v(\tau)+\bar{\xi} \bar{v}(\tau)+H(\tau, \xi, \bar{\xi}), \quad \tau \in[0,4 T], \xi \in \mathbb{C},
$$

where the real function $H$ satisfies $H(4 T, \xi, \bar{\xi})=H(0, \xi, \bar{\xi})$ and has the Taylor expansion

$$
H(\tau, \xi, \bar{\xi})=\sum_{\substack{i, j=0 \\ 2 \leq i+j \leq 3}}^{3} \frac{1}{i ! j !} h_{i j}(\tau) \xi^{i} \bar{\xi}^{j}+O\left(|\xi|^{4}\right)
$$

with $h_{i j}(4 T)=h_{i j}(0)$ and $h_{i j}=\bar{h}_{j i}$ so that $h_{i i}$ is real, while $v$ is defined by

$$
\left\{\begin{aligned}
\dot{v}-A(\tau) v & =0, \tau \in[0, T] \\
v(T)-e^{i \frac{\pi}{2}} v(0) & =0 \\
\int_{0}^{T}\langle v, v\rangle d \tau-1 & =0
\end{aligned}\right.
$$


extended on $[0,4 T]$ using the equivariance property of the normal form, i.e.,

$$
\begin{aligned}
v(\tau+T) & :=e^{i \frac{\pi}{2}} v(\tau)=i v(\tau), \\
v(\tau+2 T) & :=e^{i \pi} v(\tau)=-v(\tau), \\
v(\tau+3 T) & :=e^{i \frac{3 \pi}{2}} v(\tau)=-i v(\tau)
\end{aligned}
$$

for $\tau \in[0, T]$.

The definition of the conjugate $\bar{v}$ follows from this. These functions exist due to Lemma 2 of [25]. As usual the functions $h_{i j}$ can be found by solving appropriate BVPs, assuming that (2.1) restricted to $W^{c}(\Gamma)$ has the periodic R4 normal form from Table 1. Similar to the R3 case, it holds that

$$
h_{k l}(\tau)=h_{k l}(\tau+T)\left(e^{-i \pi / 2}\right)^{k}\left(e^{i \pi / 2}\right)^{l}
$$

for $\tau \in[0, T]$.

The adjoint eigenfunction $\varphi^{*}$ is defined by the $T$-periodic solution of (3.16), and $v^{*}$ satisfies

$$
\left\{\begin{aligned}
\dot{v}^{*}(\tau)+A^{\mathrm{T}}(\tau) v^{*} & =0, \tau \in[0, T] \\
v^{*}(T)-e^{i \frac{\pi}{2}} v^{*}(0) & =0 \\
\int_{0}^{T}\left\langle v^{*}, v\right\rangle d \tau-1 & =0
\end{aligned}\right.
$$

Similarly, we obtain $\bar{v}^{*}$.

The constant and the linear terms give the identities $\dot{u}_{0}=F\left(u_{0}\right), \dot{v}-A(\tau)=0$, and $\dot{\bar{v}}-A(\tau) \bar{v}=0$. From the $\xi^{2}$ - or $\bar{\xi}^{2}$-terms the following equation (or its complex-conjugate) follows:

$$
\dot{h}_{20}-A(\tau) h_{20}=B(\tau ; v, v) .
$$

Notice that this equation is nonsingular in the space of functions satisfying $h_{20}(T)=-h_{20}(0)$. So $h_{20}$ is given as the unique solution of the BVP

$$
\left\{\begin{aligned}
\dot{h}_{20}-A(\tau) h_{20}-B(\tau ; v, v) & =0, \tau \in[0, T] \\
h_{20}(T)+h_{20}(0) & =0 .
\end{aligned}\right.
$$

By collecting the $\xi \bar{\xi}$-terms we obtain an equation for $h_{11}$,

$$
\dot{h}_{11}-A(\tau) h_{11}=B(\tau ; v, \bar{v})-\alpha_{1} \dot{u}_{0},
$$

which is to be solved in the space of functions satisfying $h_{11}(T)=h_{11}(0)$. The Fredholm solvability condition gives exactly the same value of $\alpha_{1}$ as in (3.61). With this value of $\alpha_{1}$, $h_{11}$ is the unique solution of BVP (3.62).

The $\xi \bar{\xi}^{2}$-terms give an equation for $h_{12}$,

$$
\dot{h}_{12}-A(\tau) h_{12}=C(\tau ; v, \bar{v}, \bar{v})+B\left(\tau ; v, h_{02}\right)+2 B\left(\tau ; \bar{v}, h_{11}\right)-2 \bar{c} \bar{v}-2 \alpha_{1} \dot{\bar{v}},
$$

which is to be solved in the space of functions satisfying $h_{12}(T)=-i h_{12}(0)$. The Fredholm solvability condition implies

$$
\bar{c}=\frac{1}{2} \int_{0}^{T}\left\langle\bar{v}^{*}, C(\tau ; v, \bar{v}, \bar{v})+B\left(\tau ; v, h_{02}\right)+2 B\left(\tau ; \bar{v}, h_{11}\right)-2 \alpha_{1} A(\tau) \bar{v}\right\rangle d \tau,
$$


where $\alpha_{1}$ is defined in (3.61), and $v, h_{11}$, and $h_{02}$ are the unique solutions of the BVPs (3.63), (3.62), and the complex-conjugate of (3.65). Taking the complex-conjugate gives us the critical coefficient $c$ in the R4 normal form from Table 1.

Finally, by collecting the $\bar{\xi}^{3}$-terms we obtain an equation for $h_{03}$,

$$
\dot{h}_{03}-A(\tau) h_{03}=C(\tau ; \bar{v}, \bar{v}, \bar{v})+3 B\left(\tau ; \bar{v}, h_{02}\right)-6 d v,
$$

which is to be solved in the space of the functions satisfying $h_{03}(T)=i h_{03}(0)$. The nontrivial Fredholm solvability condition gives the value of the critical coefficient $d$ in the R4 normal form, namely,

$$
d=\frac{1}{6} \int_{0}^{T}\left\langle v^{*}, C(\tau ; \bar{v}, \bar{v}, \bar{v})+3 B\left(\tau ; \bar{v}, h_{02}\right)\right\rangle d \tau .
$$

So we finally obtain the value of

$$
A=\frac{c}{|d|}
$$

which can be used to determine the bifurcation scenario at the $\mathrm{R} 4$ resonance.

Also, in this case $v$ is not uniquely determined, since for every $\gamma \in \mathbb{C}$ with $\bar{\gamma}^{\mathrm{T}} \gamma=1, \gamma v$ is a solution. Then the adjoint eigenfunction is given by $\gamma v^{*}$, and $h_{20}$ is replaced by $\gamma^{2} h_{20}$. The normal form coefficient $c$ remains the same, but instead of $d$ we get $\bar{\gamma}^{4} d$. However, this again does not influence the bifurcation analysis since the study is determined by the above-defined $A$ for which we need only $|d|$.

3.2.6. Fold-flip bifurcation. The three-dimensional critical center manifold $W^{c}(\Gamma)$ at the LPPD bifurcation can be parameterized locally by $(\tau, \xi)$ as

$$
u=u_{0}(\tau)+\xi_{1} v_{1}(\tau)+\xi_{2} v_{2}(\tau)+H(\tau, \xi), \quad \tau \in[0,2 T], \xi=\left(\xi_{1}, \xi_{2}\right) \in \mathbb{R}^{2},
$$

where $H$ satisfies $H(2 T, \xi)=H(0, \xi)$ and has the Taylor expansion

$$
H(\tau, \xi)=\sum_{\substack{i, j=0 \\ 2 \leq i+j \leq 3}}^{3} \frac{1}{i ! j !} h_{i j}(\tau) \xi_{1}^{i} \xi_{2}^{j}+O\left(\|\xi\|^{4}\right),
$$

while the eigenfunctions $v_{1}$ and $v_{2}$ are given by

$$
\left\{\begin{aligned}
\dot{v}_{1}-A(\tau) v_{1}-F\left(u_{0}\right) & =0, \tau \in[0, T], \\
v_{1}(T)-v_{1}(0) & =0 \\
\int_{0}^{T}\left\langle v_{1}, F\left(u_{0}\right)\right\rangle d \tau & =0
\end{aligned}\right.
$$

and

$$
\left\{\begin{aligned}
\dot{v}_{2}-A(\tau) v_{2} & =0, \tau \in[0, T], \\
v_{2}(T)+v_{2}(0) & =0 \\
\int_{0}^{T}\left\langle v_{2}, v_{2}\right\rangle d \tau-1 & =0
\end{aligned}\right.
$$

with

$$
v_{1}(\tau+T):=v_{1}(\tau) \text { and } v_{2}(\tau+T):=-v_{2}(\tau) \text { for } \tau \in[0, T]
$$


The functions $v_{1}$ and $v_{2}$ exist because of Lemmas 2 and 5 of [25]. The functions $h_{i j}$ can be found by solving appropriate BVPs, assuming that (2.1) restricted to $W^{c}(\Gamma)$ has the periodic LPPD normal form in Table 1. Moreover, similarly as before, $u\left(\tau, \xi_{1}, \xi_{2}\right)=u\left(\tau+T, \xi_{1},-\xi_{2}\right)$ such that

$$
h_{i j}(\tau)=(-1)^{j} h_{i j}(\tau+T)
$$

for $\tau \in[0, T]$. As before, we will reduce all computations to the interval $[0, T]$.

To compute the coefficients of the normal form, we need the generalized eigenfunction $v_{1}$, the eigenfunction $v_{2}$, and the adjoint eigenfunctions $\varphi^{*}, v_{1}^{*}$, and $v_{2}^{*}$, defined as solution of the following BVPs:

$$
\begin{gathered}
\left\{\begin{aligned}
\dot{\varphi}^{*}+A^{\mathrm{T}}(\tau) \varphi^{*} & =0, \tau \in[0, T], \\
\varphi^{*}(T)-\varphi^{*}(0) & =0 \\
\int_{0}^{T}\left\langle\varphi^{*}, v_{1}\right\rangle d \tau-1 & =0
\end{aligned}\right. \\
\left\{\begin{aligned}
\dot{v}_{1}^{*}+A^{\mathrm{T}}(\tau) v_{1}^{*}+\varphi^{*} & =0, \tau \in[0, T], \\
v_{1}^{*}(T)-v_{1}^{*}(0) & =0 \\
\int_{0}^{T}\left\langle v_{1}^{*}, v_{1}\right\rangle d \tau & =0
\end{aligned}\right.
\end{gathered}
$$

and

$$
\left\{\begin{aligned}
\dot{v}_{2}^{*}+A^{\mathrm{T}}(\tau) v_{2}^{*} & =0, \quad \tau \in[0, T] \\
v_{2}^{*}(T)+v_{2}^{*}(0) & =0 \\
\int_{0}^{T}\left\langle v_{2}^{*}, v_{2}\right\rangle d \tau-1 & =0
\end{aligned}\right.
$$

Note that the integral conditions can be satisfied due to the spectral assumptions at the LPPD point. The following orthogonality conditions hold automatically:

$$
\begin{aligned}
\int_{0}^{T}\left\langle\varphi^{*}, F\left(u_{0}\right)\right\rangle d \tau & =\int_{0}^{T}\left\langle\varphi^{*}, v_{2}\right\rangle d \tau=\int_{0}^{T}\left\langle v_{1}^{*}, v_{2}\right\rangle d \tau \\
& =\int_{0}^{T}\left\langle v_{2}^{*}, v_{1}\right\rangle d \tau=\int_{0}^{T}\left\langle v_{2}^{*}, F\left(u_{0}\right)\right\rangle d \tau=0 .
\end{aligned}
$$

Since we have normalized the adjoint eigenfunction associated to multiplier 1 with the last generalized eigenfunction, we also have

$$
\int_{0}^{T}\left\langle v_{1}^{*}, F\left(u_{0}\right)\right\rangle d \tau=1
$$

As usual, to derive the normal form coefficients we substitute the above expansions into (2.1) and compare term by term. By collecting the constant and linear terms we get the identities $\dot{u}_{0}=F\left(u_{0}\right), \dot{v}_{1}=A(\tau) v_{1}+F\left(u_{0}\right)$, and $\dot{v}_{2}=A(\tau) v_{2}$.

By collecting the $\xi_{1}^{2}$-terms we find an equation for $h_{20}$,

$$
\dot{h}_{20}-A(\tau) h_{20}=B\left(\tau ; v_{1}, v_{1}\right)-2 a_{20} v_{1}-2 \alpha_{20} \dot{u}_{0}+2 \dot{v}_{1},
$$


which is to be solved in the space of functions satisfying $h_{20}(T)=h_{20}(0)$. In this space, the differential operator $\frac{d}{d \tau}-A(\tau)$ is singular and its null-space is spanned by $\dot{u}_{0}$. The corresponding Fredholm solvability condition implies

$$
a_{20}=\frac{1}{2} \int_{0}^{T}\left\langle\varphi^{*}, B\left(\tau ; v_{1}, v_{1}\right)+2 A(\tau) v_{1}\right\rangle d \tau
$$

With $a_{20}$ tuned in this way (3.71) is solvable for any value of parameter $\alpha_{20}$. As in the cusp of cycles case, we are free to choose parameter $\alpha_{20}$ as we want, and we take $\alpha_{20}=0$. This choice will not influence our final conclusion about possible bifurcation scenarios.

In order to make the solution of (3.71) unique, we have to fix the projection on the nullspace of the operator, more specifically in the direction of $F\left(u_{0}\right)$. Therefore, we impose the orthogonality condition with the adjoint generalized eigenfunction $v_{1}^{*}$ and obtain $h_{20}$ as the unique solution of the BVP

$$
\left\{\begin{aligned}
\dot{h}_{20}-A(\tau) h_{20}-B\left(\tau ; v_{1}, v_{1}\right)+2 a_{20} v_{1}+2 \alpha_{20} F\left(u_{0}\right)-2 A(\tau) v_{1}-2 F\left(u_{0}\right) & =0, \tau \in[0, T], \\
h_{20}(T)-h_{20}(0) & =0 \\
\int_{0}^{T}\left\langle v_{1}^{*}, h_{20}\right\rangle d \tau & =0 .
\end{aligned}\right.
$$

By collecting the $\xi_{1} \xi_{2}$-terms we obtain a singular equation for $h_{11}$,

$$
\dot{h}_{11}-A(\tau) h_{11}=B\left(\tau ; v_{1}, v_{2}\right)-b_{11} v_{2}+\dot{v}_{2},
$$

which is to be solved in the space of the functions that satisfy $h_{11}(T)=-h_{11}(0)$. The Fredholm solvability condition gives, using (3.67) and (3.70),

$$
b_{11}=\int_{0}^{T}\left\langle v_{2}^{*}, B\left(\tau ; v_{1}, v_{2}\right)+A(\tau) v_{2}\right\rangle d \tau
$$

With $b_{11}$ defined in this way, we can compute $h_{11}$ as the unique solution of the BVP

$$
\left\{\begin{aligned}
\dot{h}_{11}-A(\tau) h_{11}-B\left(\tau ; v_{1}, v_{2}\right)+b_{11} v_{2}-A(\tau) v_{2} & =0, \tau \in[0, T], \\
h_{11}(T)+h_{11}(0) & =0 \\
\int_{0}^{T}\left\langle v_{2}^{*}, h_{11}\right\rangle d \tau & =0 .
\end{aligned}\right.
$$

Collecting the $\xi_{2}^{2}$-terms gives a singular equation for $h_{02}$,

$$
\dot{h}_{02}-A(\tau) h_{02}=B\left(\tau ; v_{2}, v_{2}\right)-2 a_{02} v_{1}-2 \alpha_{02} \dot{u}_{0},
$$

where solvability gives in the standard manner

$$
a_{02}=\frac{1}{2} \int_{0}^{T}\left\langle\varphi^{*}, B\left(\tau ; v_{2}, v_{2}\right)\right\rangle d \tau
$$

So (3.72) is solvable for any value of the parameter $\alpha_{02}$. For simplicity, we take $\alpha_{02}=0$. Notice that also here, the solution of (3.72) is orthogonal to the adjoint eigenfunction $\varphi^{*}$. 
Since we have to fix the projection in the direction of eigenfunction $\dot{u}_{0}$, we define $h_{02}$ as the unique solution of

$$
\left\{\begin{aligned}
\dot{h}_{02}-A(\tau) h_{02}-B\left(\tau ; v_{2}, v_{2}\right)+2 a_{02} v_{1}+2 \alpha_{02} F\left(u_{0}\right) & =0, \tau \in[0, T], \\
h_{02}(T)-h_{02}(0) & =0 \\
\int_{0}^{T}\left\langle v_{1}^{*}, h_{02}\right\rangle d \tau & =0 .
\end{aligned}\right.
$$

Applying the Fredholm solvability conditions to the singular equations for $h_{i j}$ with $i+j=$ 3 , we obtain

$$
\begin{aligned}
a_{30}= & \frac{1}{6} \int_{0}^{T}\left\langle\varphi^{*}, C\left(\tau ; v_{1}, v_{1}, v_{1}\right)+3 B\left(\tau ; h_{20}, v_{1}\right)-6 a_{20} h_{20}\right. \\
& \left.+3\left(A(\tau) h_{20}+B\left(\tau ; v_{1}, v_{1}\right)\right)+6\left(1-\alpha_{20}\right) A(\tau) v_{1}\right\rangle d \tau-a_{20}, \\
b_{21}= & \frac{1}{2} \int_{0}^{T}\left\langle v_{2}^{*}, C\left(\tau ; v_{1}, v_{1}, v_{2}\right)+B\left(\tau ; h_{20}, v_{2}\right)+2 B\left(\tau ; h_{11}, v_{1}\right)-2 a_{20} h_{11}\right. \\
& \left.-2 b_{11} h_{11}+2\left(A(\tau) h_{11}+B\left(\tau ; v_{1}, v_{2}\right)\right)+2\left(1-\alpha_{20}\right) A(\tau) v_{2}\right\rangle d \tau-b_{11}, \\
a_{12}= & \frac{1}{2} \int_{0}^{T}\left\langle\varphi^{*}, C\left(\tau ; v_{1}, v_{2}, v_{2}\right)+B\left(\tau ; h_{02}, v_{1}\right)+2 B\left(\tau ; h_{11}, v_{2}\right)-2 b_{11} h_{02}\right. \\
& \left.-2 a_{02} h_{20}+A(\tau) h_{02}+B\left(\tau ; v_{2}, v_{2}\right)-2 \alpha_{02} A(\tau) v_{1}\right\rangle d \tau-a_{02}, \\
b_{03}= & \frac{1}{6} \int_{0}^{T}\left\langle v_{2}^{*}, C\left(\tau ; v_{2}, v_{2}, v_{2}\right)+3 B\left(\tau ; h_{02}, v_{2}\right)-6 a_{02} h_{11}-6 \alpha_{02} A(\tau) v_{2}\right\rangle d \tau .
\end{aligned}
$$

4. Implementation issues. Numerical implementation of the formulas derived in section 3 requires the evaluation of integrals of scalar functions over $[0, T]$ and the solution of nonsingular linear BVPs with integral constraints. Such tasks can be carried out as in the standard continuation software such as AUTO [17], CONTENT [31], and MATCONT [14]. In these software packages, periodic solutions to (1.1) are computed with the method of orthogonal collocation with piecewise polynomials applied to properly formulated BVPs.

The standard BVP for the periodic solutions is formulated on the unit interval $[0,1]$, so that the period $T$ becomes a parameter, and it involves an integral phase condition,

$$
\left\{\begin{aligned}
\dot{x}(\tau)-T f(x(\tau), \alpha) & =0, \tau \in[0,1] \\
x(0)-x(1) & =0 \\
\int_{0}^{1}\langle x(\tau), \dot{\xi}(\tau)\rangle d \tau & =0
\end{aligned}\right.
$$

where $\xi$ is a previously calculated periodic solution to a nearby problem, rescaled to $[0,1]$.

In the orthogonal collocation method [5], problem (4.1) is replaced by the following discretization:

$$
\left\{\begin{array}{r}
\sum_{j=0}^{m} x_{i, j} \dot{\ell}_{i, j}\left(\zeta_{i, k}\right)-T f\left(\sum_{j=0}^{m} x_{i, j} \ell_{i, j}\left(\zeta_{i, k}\right), \alpha\right)=0 \\
x_{0,0}-x_{N-1, m}=0 \\
\sum_{i=0}^{N-1} \sum_{j=0}^{m-1} \sigma_{i, j}\left\langle x_{i, j}, \dot{\xi}_{i, j}\right\rangle+\sigma_{N, 0}\left\langle x_{N, 0}, \dot{\xi}_{N, 0}\right\rangle=0
\end{array}\right.
$$


The points $x_{i, j}$ form the approximation of $x(\tau)$ with $m+1$ equidistant mesh points

$$
\tau_{i, j}=\tau_{i}+\frac{j}{m}\left(\tau_{i+1}-\tau_{i}\right), \quad j=0,1, \ldots, m,
$$

in each of the $N$ intervals $\left[\tau_{i}, \tau_{i+1}\right]$, where $0=\tau_{0}<\tau_{1}<\cdots<\tau_{N}=1$.

The $\ell_{i, j}(\tau)$ 's are the Lagrange basis polynomials, while the points $\zeta_{i, j}(j=1, \ldots, m)$ are Gauss points [12], i.e., the roots of the Legendre polynomial of degree $m$, all relative to the interval $\left[\tau_{i}, \tau_{i+1}\right]$.

With this choice of collocation points $\zeta_{i, j}$, the approximation error at the mesh points has order of accuracy $m+1,\left\|x\left(\tau_{i, j}\right)-x_{i, j}\right\|=\mathcal{O}\left(h^{m+1}\right)$, where $h=\max _{i=1,2, \ldots, N}\left\{t_{i}\right\}, t_{i}=\tau_{i}-\tau_{i-1}$, while for the coarse mesh points $\tau_{i}$ the error has order of accuracy $2 m,\left\|x\left(\tau_{i}\right)-x_{i, 0}\right\|=\mathcal{O}\left(h^{2 m}\right)$ ("superconvergence").

The integration weight $\sigma_{i, j}$ of $\tau_{i, j}$ is given by $w_{j+1} t_{i+1}$ for $0 \leq i \leq N-1$ and $0<$ $j<m$. For $i=0, \ldots, N-2$, the integration weight of $\tau_{i, m}\left(\tau_{i, m}=\tau_{i+1,0}\right)$ is given by $\sigma_{i, m}=w_{m+1} t_{i+1}+w_{1} t_{i+2}$, and the integration weights of $\tau_{0}$ and $\tau_{N}$ are given by $w_{1} t_{1}$ and $w_{m+1} t_{N}$, respectively. In the above expressions, $w_{j+1}$ is the Lagrange quadrature coefficient.

4.1. Discretization symbols. It is convenient to discretize all computed functions using the same mesh as in (4.2). For a given vector function $\eta \in \mathcal{C}^{1}\left([0,1], \mathbb{R}^{n}\right)$ we consider three different discretizations:

- $\eta_{M} \in \mathbb{R}^{(N m+1) n}$, the vector of the function values at the mesh points;

- $\eta_{C} \in \mathbb{R}^{N m n}$, the vector of the function values at the collocation points;

- $\eta_{W}=\left[\begin{array}{l}\eta_{W_{1}} \\ \eta_{W_{2}}\end{array}\right] \in \mathbb{R}^{N m n} \times \mathbb{R}^{n}$, where $\eta_{W_{1}}$ is the vector of the function values at the collocation points multiplied by the Gauss-Legendre weights and the lengths of the corresponding mesh intervals, and $\eta_{W_{2}}=\eta(0)$.

Formally, we also introduce the structured sparse matrix $L_{C \times M}$ that converts a vector $\eta_{M}$ of function values at the mesh points into a vector $\eta_{C}$ of its values at the collocation points, namely, $\eta_{C}=L_{C \times M} \eta_{M}$. This matrix is never formed explicitly; its entries are the $\ell_{i, j}\left(\zeta_{i, k}\right)$ coefficients in (4.2). We also need a matrix $A_{C \times M}$ such that $A_{C \times M} \eta_{M}=(A(t) \eta(t))_{C}$. Again this matrix need not be formed explicitly. On the other hand, we do need the matrix $(D-$ $T A(t))_{C \times M}$ explicitly; it is defined by $(D-T A(t))_{C \times M} \eta_{M}=(\dot{\eta}(t)-T A(t) \eta(t))_{C}$. Finally, let the tensors $B_{C \times M \times M}$ and $C_{C \times M \times M \times M}$ be defined by $B_{C \times M \times M} \eta_{1 M} \eta_{2 M}=\left(B\left(t ; \eta_{1}(t), \eta_{2}(t)\right)\right)_{C}$ and

$$
C_{C \times M \times M \times M} \eta_{1 M} \eta_{2 M} \eta_{3 M}=\left(C\left(t ; \eta_{1}(t), \eta_{2}(t), \eta_{3}(t)\right)\right)_{C}
$$

for all $\eta_{i} \in \mathcal{C}^{1}\left([0,1], \mathbb{R}^{n}\right)$. (These tensors are not formed explicitly.)

Let $f(t), g(t) \in \mathcal{C}^{0}([0,1], \mathbb{R})$ be two scalar functions. Then the integral $\int_{0}^{1} f(t) d t$ is represented by $\sum_{i=0}^{N-1} \sum_{j=1}^{m} \omega_{j}\left(f_{C}\right)_{i, j} t_{i+1}=\sum_{i=0}^{N-1} \sum_{j=1}^{m}\left(f_{W_{1}}\right)_{i, j}$, where $\left(f_{C}\right)_{i, j}=f\left(\zeta_{i, j}\right)$ and $\omega_{j}$ is the Gauss-Legendre quadrature coefficient. The integral $\int_{0}^{1} f(t) g(t) d t$ is approximated with Gauss-Legendre by $f_{W_{1}}^{\mathrm{T}} g_{C} \approx f_{W_{1}}^{\mathrm{T}} L_{C \times M} g_{M}$, where equality holds if $g(t)$ is a piecewise polynomial of degree $m$ or less on the given mesh. For vector functions $f(t), g(t) \in \mathcal{C}^{0}\left([0,1], \mathbb{R}^{n}\right)$, the integral $\int_{0}^{1}\langle f(t), g(t)\rangle d t$ is formally approximated by the same expression: $f_{W_{1}}^{\mathrm{T}} g_{C} \approx$ $f_{W_{1}}^{\mathrm{T}} L_{C \times M} g_{M}$, where again we have equality if $g(t)$ is a piecewise polynomial of degree $m$ or less on the given mesh. Concerning the accuracy of the quadrature formulas, we first note that accuracy is not an important issue for the phase integral in (4.1), as this equation selects 
only a specific solution from the continuum of solutions obtained by phase shifts. Similarly, the discretization of the normalization integrals does not affect the inherent accuracy, including superconvergence at the main mesh points $\tau_{i}$ of the solution of the discretized BVP. Discretization of integrals, as specified above, follows the standard Gauss quadrature error, which has order of accuracy $2 m$ if, as mentioned before, the function $g(t)$ is a piecewise polynomial of degree $m$ or less on the given mesh and if $f(t)$ is sufficiently smooth (in a piecewise sense). Otherwise, still assuming sufficient piecewise smoothness, the order of accuracy of the numerical integrals is $m+1$ if $m$ is odd, and $m+2$ if $m$ is even. In particular, for the often used choice $m=4$, the integrals would then have order of accuracy 6 .

In section 3 we derived the coefficients of the critical normal forms in Table 1 and the functions needed for their computation using the coordinate $\tau \in[0, T]$. Regarding the implementation, in all cases we will rescale to the interval $[0,1]$. Therefore, define $u_{1}(t)=u_{0}(T t)=u_{0}(\tau)$ for $t \in[0,1]$, where $u_{0}$ was defined in section 2 . In general, vector-functions with the rescaled argument will have an extra lower index 1 . Note that the multilinear forms $A, B, C, D$, and $E$ are computed by means of the MATLAB symbolic toolbox.

4.2. Cusp of cycles bifurcation. The linear BVPs (3.3), (3.4), and (3.5) defining the generalized eigenfunction, the adjoint eigenfunction, and the generalized adjoint eigenfunctions, respectively, are to be replaced by the rescaled problems

$$
\left\{\begin{aligned}
\dot{v}_{1}(t)-T A(t) v_{1}(t)-T F\left(u_{1}(t)\right) & =0, t \in[0,1], \\
v_{1}(1)-v_{1}(0) & =0 \\
\int_{0}^{1}\left\langle v_{1}(t), F\left(u_{1}(t)\right)\right\rangle d t & =0
\end{aligned}\right.
$$

with $v(\tau)=v_{1}(\tau / T)$,

$$
\left\{\begin{aligned}
\dot{\varphi}_{1}^{*}(t)+T A^{\mathrm{T}}(t) \varphi_{1}^{*}(t) & =0, \quad t \in[0,1] \\
\varphi_{1}^{*}(1)-\varphi_{1}^{*}(0) & =0 \\
\int_{0}^{1}\left\langle\varphi_{1}^{*}(t), v_{1}(t)\right\rangle d t-1 & =0
\end{aligned}\right.
$$

where $\varphi^{*}(\tau)=\varphi_{1}^{*}(\tau / T) / T$, and

$$
\left\{\begin{aligned}
\dot{v}_{1}^{*}(t)+T A^{\mathrm{T}}(t) v_{1}^{*}(t)+T \varphi_{1}^{*}(t) & =0, \quad t \in[0,1], \\
v_{1}^{*}(1)-v_{1}^{*}(0) & =0 \\
\int_{0}^{1}\left\langle v_{1}^{*}(t), v_{1}(t)\right\rangle d t & =0
\end{aligned}\right.
$$

with $v^{*}(\tau)=v_{1}^{*}(\tau / T) / T$. We then still need $h_{2,1}(t)$, which is the unique solution of the rescaled BVP (3.12), i.e.,

$$
\left\{\begin{aligned}
\dot{h}_{2,1}(t)-T A(t) h_{2,1}(t)-T B\left(t ; v_{1}(t), v_{1}(t)\right) & \\
-2 T A(t) v_{1}(t)-2 T F\left(u_{1}(t)\right) & =0, \quad t \in[0,1], \\
h_{2,1}(1)-h_{2,1}(0) & =0 \\
\int_{0}^{1}\left\langle v_{1}^{*}(t), h_{2,1}(t)\right\rangle d t & =0
\end{aligned}\right.
$$

where $h_{2}(\tau)=h_{2,1}(\tau / T)$, to compute the normal form coefficient $c$ via

$$
\begin{aligned}
c=\frac{1}{6} \int_{0}^{1}\left\langle\varphi_{1}^{*}(t), 3 A(t) h_{2,1}(t)+3 B\left(t ; v_{1}(t), v_{1}(t)\right)\right. \\
\left.+6 A(t) v_{1}(t)+3 B\left(t ; h_{2,1}(t), v_{1}(t)\right)+C\left(t ; v_{1}(t), v_{1}(t), v_{1}(t)\right)\right\rangle d t .
\end{aligned}
$$


We now give the matrix approximations. We compute $v_{1 M}$ by solving the discretization of (4.3), i.e.,

$$
\left[\begin{array}{cc}
(D-T A(t))_{C \times M} & p \\
\delta_{0}-\delta_{1} & 0
\end{array}\right]\left[\begin{array}{c}
v_{1 M} \\
a_{1}
\end{array}\right]=\left[\begin{array}{c}
T f_{C} \\
0_{n \times 1} \\
0
\end{array}\right],
$$

where $a_{1}$ equals zero since the $M \times M$ upper left part of the big matrix is singular, $g(t)=$ $F\left(u_{1}(t)\right)$, and $p$ is obtained by solving the system

$$
\left[\begin{array}{ll}
p^{\mathrm{T}} & a_{2}
\end{array}\right]\left[\begin{array}{cc}
(D-T A(t))_{C \times M} & r_{1} \\
\delta_{0}-\delta_{1} & 0
\end{array}\right]=\left[\begin{array}{ll}
0_{M \times 1} & 1
\end{array}\right]
$$

where $r_{1}$ and $r_{2}$ are any vectors which make the big matrix in (4.8) nonsingular. Here, $a_{2}=0$ and $p$ is then the left null-vector of $\left[\begin{array}{c}(D-T A(t))_{C \times M} \\ \delta_{0}-\delta_{1}\end{array}\right]$; in (4.7) the normalized $p$ is used. This technique guarantees that we always deal with nonsingular systems.

We will compute $\varphi_{1 W}^{*}$ instead of $\varphi_{1 M}^{*}$ since $\varphi_{1 W}^{*}$ can be calculated by a system very similar to (4.7). Formally, the computation of $\varphi_{1 W}^{*}$ is based on Proposition B.1 from the supplementary files, i.e., since $\varphi \in \operatorname{Ker}\left(\phi_{2}\right)$, with $\phi_{2}$ defined in Proposition B.1. We need

$$
\left(\varphi_{1}^{*}\right)_{W}^{\mathrm{T}}\left[\begin{array}{c}
(D-T A(t))_{C \times M} \\
\delta_{0}-\delta_{1}
\end{array}\right]=0 .
$$

Therefore, $\varphi_{1 W}^{*}$ can be obtained by solving

$$
\left[\left(\varphi_{1}^{*}\right)_{W}^{\mathrm{T}} \quad a\right]\left[\begin{array}{cc}
(D-T A(t))_{C \times M} & p \\
\delta_{0}-\delta_{1} & 0
\end{array}\right]=\left[\begin{array}{ll}
0_{M \times 1} & 1
\end{array}\right]
$$

where $a$ equals zero and $q$ is the normalized right null-vector of $\left[\begin{array}{c}(D-T A(t))_{C \times M} \\ \delta_{0}-\delta_{1}\end{array}\right]$. We then approximate $I=\int_{0}^{1}\left\langle\varphi_{1}^{*}(t), v_{1}(t)\right\rangle d t$ by $I_{1}=\left(\varphi_{1}^{*}\right)_{W_{1}}^{\mathrm{T}} L_{C \times M} v_{1 M} \cdot \varphi_{1 W}^{*}$ is then rescaled to ensure that $I_{1}=1$.

It is more efficient to compute $v_{1 W}^{*}$ instead of $v_{1 M}^{*}$, since $v_{1}^{*}$ will be used only to compute integrals of the form $\int_{0}^{1}\left\langle v_{1}^{*}(t), \zeta(t)\right\rangle d t$. From Proposition B.3 we can conclude that

$$
\left\langle\left[\begin{array}{c}
v_{1}^{*} \\
v_{1}^{*}(0)
\end{array}\right],\left[\begin{array}{c}
\dot{h}-T A(t) h \\
h(0)-h(1)
\end{array}\right]\right\rangle=-\left\langle\left[\begin{array}{c}
-T \varphi_{1}^{*} \\
0
\end{array}\right],\left[\begin{array}{l}
h \\
0
\end{array}\right]\right\rangle
$$

for all appropriate functions $h$, such that $v_{1}^{*}$ can be obtained by solving

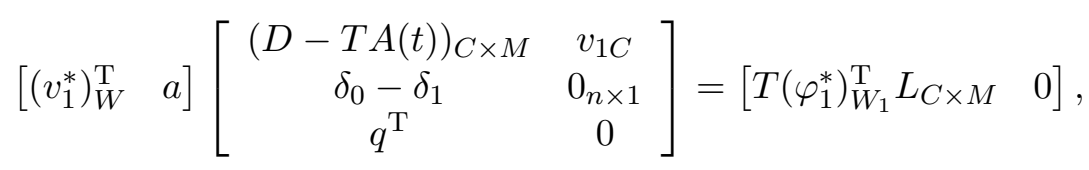

where $a$ equals zero and $p$ is defined in (4.8). 
Next, $\left(h_{2,1}\right)_{M}$ is found by solving the discretization of (4.5), namely,

$$
\left[\begin{array}{cc}
(D-T A(t))_{C \times M} & p \\
\delta_{0}-\delta_{1} & 0
\end{array}\right]\left[\begin{array}{c}
h_{2,1 M} \\
a
\end{array}\right]=\left[\begin{array}{c}
\mathrm{R} \\
0_{n \times 1} \\
0
\end{array}\right],
$$

with $\mathrm{R}=T B_{C \times M \times M} v_{1 M} v_{1 M}+2 T A_{C \times M} v_{1 M}+2 T g_{C}, a=0$, and $p$ defined above.

Finally, (4.6) is approximated by

$$
\begin{aligned}
c= & \frac{1}{6}\left(\varphi_{1}^{*}\right)_{W_{1}}^{\mathrm{T}}\left(3 A_{C \times M} h_{2,1 M}+3 B_{C \times M \times M} v_{1 M} v_{1 M}\right. \\
& \left.+6 A_{C \times M} v_{1 M}+3 B_{C \times M \times M} h_{2,1 M} v_{1 M}+C_{C \times M \times M \times M} v_{1 M} v_{1 M} v_{1 M}\right) .
\end{aligned}
$$

Note that since we have a CPC bifurcation, the quadratic coefficient of the $\xi$-equation in the corresponding normal form has to be equal to zero. MATCONT makes an extra check whether this coefficient [30], determined by

$$
b=\frac{1}{2}\left(\varphi_{1}^{*}\right)_{W_{1}}^{\mathrm{T}}\left(B_{C \times M \times M} v_{1 M} v_{1 M}+2 A_{C \times M} v_{1 M}\right),
$$

is indeed small enough.

4.3. GPD bifurcation. The linear BVPs (3.15), (3.16), and (3.17) defining the eigenfunction associated to multiplier -1 and the adjoint eigenfunctions become the following rescaled problems:

$$
\left\{\begin{aligned}
\dot{v}_{1}(t)-T A(t) v_{1}(t) & =0, t \in[0,1] \\
v_{1}(1)+v_{1}(0) & =0 \\
\int_{0}^{1}\left\langle v_{1}(t), v_{1}(t)\right\rangle d t-1 & =0
\end{aligned}\right.
$$

where $v(\tau)=v_{1}(\tau / T) / \sqrt{T}$,

$$
\left\{\begin{aligned}
\dot{\varphi}_{1}^{*}(t)+T A^{\mathrm{T}}(t) \varphi_{1}^{*}(t) & =0, t \in[0,1] \\
\varphi_{1}^{*}(1)-\varphi_{1}^{*}(0) & =0 \\
\int_{0}^{1}\left\langle\varphi_{1}^{*}(t), F\left(u_{1}(t)\right)\right\rangle d t-1 & =0
\end{aligned}\right.
$$

with $\varphi^{*}(\tau)=\varphi_{1}^{*}(\tau / T) / T$, and

$$
\left\{\begin{aligned}
\dot{v}_{1}^{*}(t)+T A^{\mathrm{T}}(t) v_{1}^{*}(t) & =0, t \in[0,1] \\
v_{1}^{*}(1)+v_{1}^{*}(0) & =0 \\
\int_{0}^{1}\left\langle v_{1}^{*}(t), v_{1}(t)\right\rangle d t-1 & =0
\end{aligned}\right.
$$

with $v^{*}(\tau)=v_{1}^{*}(\tau / T) / \sqrt{T}$.

Let $h_{2,1}(t)$ be the unique solution of the rescaled version of BVP (3.20), i.e.,

$$
\left\{\begin{aligned}
\dot{h}_{2,1}(t)-T A(t) h_{2,1}(t)-T B\left(t ; v_{1}(t), v_{1}(t)\right)+2 \alpha_{1,1} T F\left(u_{1}(t)\right) & =0, \quad t \in[0,1], \\
h_{2,1}(1)-h_{2,1}(0) & =0, \\
\int_{0}^{1}\left\langle\varphi_{1}^{*}(t), h_{2,1}(t)\right\rangle d t & =0,
\end{aligned}\right.
$$


with $h_{2}(\tau)=h_{2,1}(\tau / T) / T$, and $h_{3,1}(t)$ be the unique solution of the rescaled version of BVP (3.23), i.e.,

$$
\left\{\begin{aligned}
\dot{h}_{3,1}(t)-T A(t) h_{3,1}(t)-T C\left(t ; v_{1}(t), v_{1}(t), v_{1}(t)\right) & \\
-3 T B\left(t ; v_{1}(t), h_{2,1}(t)\right)+6 \alpha_{1,1} T A(t) v_{1}(t) & =0, \quad t \in[0,1], \\
h_{3,1}(1)+h_{3,1}(0) & =0 \\
\int_{0}^{1}\left\langle v_{1}^{*}(t), h_{3,1}(t)\right\rangle d t & =0
\end{aligned}\right.
$$

with $h_{3}(\tau)=h_{3,1}(\tau / T) /(\sqrt{T} T)$, where now

$$
\alpha_{1,1}=\frac{1}{2} \int_{0}^{1}\left\langle\varphi_{1}^{*}(t), B\left(t ; v_{1}(t), v_{1}(t)\right)\right\rangle d t
$$

and $\alpha_{1,1}=T \alpha_{1}$.

The coefficient $h_{4,1}(t)$ is obtained as the unique solution of the rescaled BVP

$$
\left\{\begin{aligned}
\dot{h}_{4,1}(t)-T A(t) h_{4,1}(t)-T D\left(t ; v_{1}(t), v_{1}(t), v_{1}(t), v_{1}(t)\right) & \\
-6 T C\left(t ; v_{1}(t), v_{1}(t), h_{2,1}(t)\right)-3 T B\left(t ; h_{2,1}(t), h_{2,1}(t)\right) & \\
-4 T B\left(t ; v_{1}(t), h_{3,1}(t)\right)+12 \alpha_{1,1} T\left(A(t) h_{2,1}(t)+B\left(t ; v_{1}(t), v_{1}(t)\right)\right. & \\
-2 \alpha_{1,1} F\left(u_{1}(t)\right)+24 \alpha_{2,1} T F\left(u_{1}(t)\right) & =0, t \in[0,1], \\
h_{4,1}(1)-h_{4,1}(0) & =0, \\
\int_{0}^{1}\left\langle\varphi_{1}^{*}(t), h_{4,1}(t)\right\rangle d t & =0,
\end{aligned}\right.
$$

with $h_{4}(\tau)=h_{4,1}(\tau / T) / T^{2}$, and where

$$
\begin{aligned}
\alpha_{2,1}= & \frac{1}{24} \int_{0}^{1}\left\langle\varphi_{1}^{*}(t), D\left(t ; v_{1}(t), v_{1}(t), v_{1}(t), v_{1}(t)\right)+6 C\left(t ; v_{1}(t), v_{1}(t), h_{2,1}(t)\right)\right. \\
& +3 B\left(t ; h_{2,1}(t), h_{2,1}(t)\right)+4 B\left(t ; v_{1}(t), h_{3,1}(t)\right)-12 \alpha_{1,1}\left(A(t) h_{2,1}(t)\right. \\
& \left.\left.+B\left(t ; v_{1}(t), v_{1}(t)\right)\right)\right\rangle d t+\alpha_{1,1}^{2}
\end{aligned}
$$

with $\alpha_{2,1}=T^{2} \alpha_{2}$.

Finally, we can write the critical coefficient as

$$
\begin{aligned}
e= & \frac{1}{120 T^{2}} \int_{0}^{1}\left\langle v_{1}^{*}(t), E\left(t ; v_{1}(t), v_{1}(t), v_{1}(t), v_{1}(t), v_{1}(t)\right)+10 D\left(t ; v_{1}(t), v_{1}(t), v_{1}(t), h_{2,1}(t)\right)\right. \\
& +15 C\left(t ; v_{1}(t), h_{2,1}(t), h_{2,1}(t)\right)+10 C\left(t ; v_{1}(t), v_{1}(t), h_{3,1}(t)\right)+10 B\left(t ; h_{2,1}(t), h_{3,1}(t)\right) \\
& \left.+5 B\left(t ; v_{1}(t), h_{4,1}(t)\right)-120 \alpha_{2,1} A(t) v_{1}(t)-20 \alpha_{1,1} A(t) h_{3,1}(t)\right\rangle d t .
\end{aligned}
$$

We now come to the implementation details in MATCONT. We compute $v_{1 M}$ by solving

$$
\left[\begin{array}{cc}
(D-T A(t))_{C \times M} & p_{1} \\
\delta_{0}+\delta_{1} & 0
\end{array}\right]\left[\begin{array}{c}
v_{1 M} \\
a_{1}
\end{array}\right]=\left[\begin{array}{c}
0_{C \times 1} \\
0_{n \times 1} \\
1
\end{array}\right],
$$

with $p_{1}$ and $q_{1}$ the normalized solutions of

$$
\left[\begin{array}{cc}
(D-T A(t))_{C \times M} & r_{1} \\
\delta_{0}+\delta_{1} & 0
\end{array}\right]\left[\begin{array}{l}
q_{1} \\
a_{2}
\end{array}\right]=\left[\begin{array}{c}
0_{C \times 1} \\
0_{n \times 1} \\
1
\end{array}\right]
$$


and

$$
\left[\begin{array}{ll}
p_{1}^{\mathrm{T}} & a_{3}
\end{array}\right]\left[\begin{array}{cc}
(D-T A(t))_{C \times M} & r_{1} \\
\delta_{0}+\delta_{1} & 0
\end{array}\right]=\left[\begin{array}{ll}
0_{M \times 1} & 1
\end{array}\right],
$$

where $r_{1}$ and $r_{2}$ are random vectors. Every $a_{i}$ equals zero. $v_{1 M}$ is then uniquely determined by the normalization $\sum_{i=0}^{N-1} \sum_{j=0}^{m} \sigma_{j}\left\langle\left(v_{1 M}\right)_{i, j},\left(v_{1 M}\right)_{i, j}\right\rangle=1$, where $\sigma_{j}$ is the Lagrange quadrature coefficient.

Equation (4.9) is used for the computation of $\varphi_{1 W}^{*}$. We approximate $I=\int_{0}^{1}\left\langle\varphi_{1}^{*}(t), F\left(u_{1}(t)\right)\right\rangle d t$ by $I_{1}=\left(\varphi_{1}^{*}\right)_{W_{1}}^{\mathrm{T}} g_{C}$ and normalize $\varphi_{1 W}^{*}$ to ensure that $I_{1}=1$.

The discretization of (4.12) can be computed with the same matrix as in (4.13) (see the antiperiodic version of Proposition B.1 in the supplementary files),

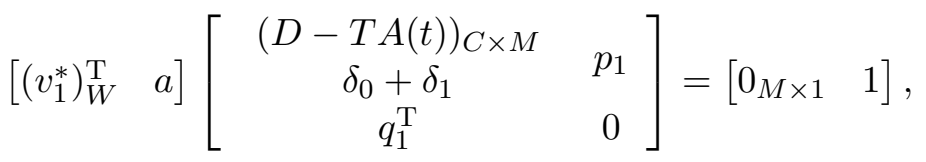

where $a=0$. We approximate $I=\int_{0}^{1}\left\langle v_{1}^{*}(t), v_{1}(t)\right\rangle d t$ by $I_{1}=\left(v_{1}^{*}\right)_{W_{1}}^{\mathrm{T}} L_{C \times M} v_{1 M} . \quad v_{1 W}^{*}$ is rescaled to ensure that $I_{1}=1$.

This then makes it possible to compute the expression for $\alpha_{1,1}$, namely,

$$
\alpha_{1,1}=\frac{1}{2}\left(\varphi_{1}^{*}\right)_{W_{1}}^{\mathrm{T}} B_{C \times M \times M} v_{1 M} v_{1 M} .
$$

Now, $h_{2,1}(t), h_{3,1}(t)$, and $h_{4,1}(t)$ are found by solving the systems

$$
\begin{gathered}
{\left[\begin{array}{cc}
(D-T A(t))_{C \times M} & p \\
\delta_{0}-\delta_{1} & 0
\end{array}\right]\left[\begin{array}{c}
h_{2,1 M} \\
a_{1}
\end{array}\right]=\left[\begin{array}{c}
T B_{C \times M \times M} v_{1 M} v_{1 M}-2 \alpha_{1,1} T g_{C} \\
0_{n \times 1} \\
0
\end{array}\right],} \\
\left(\varphi_{1}^{*}\right)_{W_{1}}^{\mathrm{T}} L_{C \times M} \\
{\left[\begin{array}{cc}
(D-T A(t))_{C \times M} & p_{1} \\
\delta_{0}+\delta_{1} & \\
\left(v_{1}^{*}\right)_{W_{1}}^{\mathrm{T}} L_{C \times M} & 0
\end{array}\right]\left[\begin{array}{c}
h_{3,1 M} \\
a_{2}
\end{array}\right]=\left[\begin{array}{c}
\mathrm{R} \\
0_{n \times 1} \\
0
\end{array}\right],}
\end{gathered}
$$

with

$$
\mathrm{R}=T C_{C \times M \times M \times M} v_{1 M} v_{1 M} v_{1 M}+3 T B_{C \times M \times M} v_{1 M} h_{2,1 M}-6 \alpha_{1,1} T A_{C \times M} v_{1 M},
$$

and

$$
\left[\begin{array}{cc}
(D-T A(t))_{C \times M} & p \\
\delta_{0}-\delta_{1} & 0
\end{array}\right]\left[\begin{array}{c}
h_{4,1 M} \\
a_{3}
\end{array}\right]=\left[\begin{array}{c}
\mathrm{R} \\
0_{n \times 1} \\
0
\end{array}\right],
$$

with

$$
\begin{aligned}
\mathrm{R}= & T D_{C \times M \times M \times M \times M} v_{1 M} v_{1 M} v_{1 M} v_{1 M}+6 T C_{C \times M \times M \times M} v_{1 M} v_{1 M} h_{2,1 M} \\
& +3 T B_{C \times M \times M} h_{2,1 M} h_{2,1 M}+4 T B_{C \times M \times M} v_{1 M} h_{3,1 M} \\
& -12 \alpha_{1,1} T\left(A_{C \times M} h_{2,1 M}+B_{C \times M \times M} v_{1 M} v_{1 M}-2 \alpha_{1,1} g_{C}\right)-24 \alpha_{2,1} T g_{C}
\end{aligned}
$$


and

$$
\begin{aligned}
\alpha_{2,1}= & \frac{1}{24}\left(\varphi_{1}^{*}\right)_{W_{1}}^{\mathrm{T}}\left(D_{C \times M \times M \times M \times M} v_{1 M} v_{1 M} v_{1 M} v_{1 M}+6 C_{C \times M \times M \times M} v_{1 M} v_{1 M} h_{2,1 M}\right. \\
& +3 B_{C \times M \times M} h_{2,1 M} h_{2,1 M}+4 B_{C \times M \times M} v_{1 M} h_{3,1 M}-12 \alpha_{1,1}\left(A_{C \times M} h_{2,1 M}\right. \\
& \left.\left.+B_{C \times M \times M} v_{1 M} v_{1 M}\right)\right)+\alpha_{1,1}^{2} .
\end{aligned}
$$

Here, $q$ is the normalized right null-vector and $p$ the normalized left null-vector of the $M \times M$ matrix corresponding with the $T$-periodic boundary condition, as before. In what follows, $p$, $q, p_{1}$, and $q_{1}$ will denote the previously defined null-vectors.

Now, we have all ingredients for the computation of the normal form coefficient

$$
\begin{aligned}
e= & \frac{1}{120 T^{2}}\left(v_{1}^{*}\right)_{W_{1}}^{\mathrm{T}}\left(E_{C \times M \times M \times M \times M \times M} v_{1 M} v_{1 M} v_{1 M} v_{1 M} v_{1 M}\right. \\
& +10 D_{C \times M \times M \times M \times M} v_{1 M} v_{1 M} v_{1 M} h_{2,1 M}+15 C_{C \times M \times M \times M} v_{1 M} h_{2,1 M} h_{2,1 M} \\
& +10 C_{C \times M \times M \times M} v_{1 M} v_{1 M} h_{3,1 M}+10 B_{C \times M \times M} h_{2,1 M} h_{3,1 M} \\
& \left.+5 B_{C \times M \times M} v_{1 M} h_{4,1 M}-120 \alpha_{2,1} A_{C \times M} v_{1 M}-20 \alpha_{1,1} A_{C \times M} h_{3,1 M}\right) .
\end{aligned}
$$

Note that since we are in the GPD case, the cubic coefficient of the $\xi$-equation in the corresponding normal form has to vanish. MATCONT makes an extra check whether this coefficient, computed as explained in [30] via

$$
c=\frac{1}{3 T}\left(v_{1}^{*}\right)_{W_{1}}^{\mathrm{T}}\left(C_{C \times M \times M} v_{1 M} v_{1 M} v_{1 M}+3 B_{C \times M \times M} v_{1 M} h_{2,1 M}-6 \alpha_{1,1} A_{C \times M} v_{1 M}\right),
$$

is indeed small enough.

4.4. $\mathrm{CH}$ bifurcation. The linear BVPs (3.26) and (3.27) defining the eigenfunction associated to the complex multiplier and the adjoint eigenfunctions are substituted by the rescaled problems

$$
\left\{\begin{aligned}
\dot{v}_{1}(t)-T A(t) v_{1}(t)+i \omega T v_{1}(t) & =0, t \in[0,1] \\
v_{1}(1)-v_{1}(0) & =0 \\
\int_{0}^{1}\left\langle v_{1}(t), v_{1}(t)\right\rangle d t-1 & =0
\end{aligned}\right.
$$

with $v(\tau)=v_{1}(\tau / T) / \sqrt{T},(4.11)$, and

$$
\left\{\begin{aligned}
\dot{v}_{1}^{*}(t)+T A^{\mathrm{T}}(t) v_{1}^{*}(t)+i \omega T v_{1}^{*}(t) & =0, \quad t \in[0,1] \\
v_{1}^{*}(1)-v_{1}^{*}(0) & =0 \\
\int_{0}^{1}\left\langle v_{1}^{*}(t), v_{1}(t)\right\rangle d t-1 & =0
\end{aligned}\right.
$$

where $v^{*}(\tau)=v_{1}^{*}(\tau / T) / \sqrt{T}$.

The second order coefficients of the center manifold are now defined by the corresponding rescaled problems

$$
\left\{\begin{aligned}
\dot{h}_{20,1}(t)-T A(t) h_{20,1}(t)+2 i \omega T h_{20,1}(t)-T B\left(t ; v_{1}(t), v_{1}(t)\right) & =0, t \in[0,1] \\
h_{20,1}(1)-h_{20,1}(0) & =0
\end{aligned}\right.
$$

where $h_{20}(\tau)=h_{20,1}(\tau / T) / T$, and

$$
\left\{\begin{aligned}
\dot{h}_{11,1}(t)-T A(t) h_{11,1}(t)-T B\left(t ; v_{1}(t), \bar{v}_{1}(t)\right)+\alpha_{1,1} T F\left(u_{1}(t)\right) & =0, t \in[0,1], \\
h_{11,1}(1)-h_{11,1}(0) & =0 \\
\int_{0}^{1}\left\langle\varphi_{1}^{*}(t), h_{11,1}(t)\right\rangle d t & =0
\end{aligned}\right.
$$


with $h_{11}(\tau)=h_{11,1}(\tau / T) / T$, where

$$
\alpha_{1,1}=\int_{0}^{1}\left\langle\varphi_{1}^{*}(t), B\left(t ; v_{1}(t), \bar{v}_{1}(t)\right)\right\rangle d t,
$$

with $\alpha_{1,1}=T \alpha_{1}$.

Now, we can compute

$$
\begin{aligned}
c_{1}= & -\frac{i}{2} \int_{0}^{1}\left\langle v_{1}^{*}(t), C\left(t ; v_{1}(t), v_{1}(t), \bar{v}_{1}(t)\right)+2 B\left(t ; v_{1}(t), h_{11,1}(t)\right)+B\left(t ; \bar{v}_{1}(t), h_{20,1}(t)\right)\right. \\
& \left.-2 \alpha_{1,1} A(t) v_{1}(t)\right\rangle d t+\alpha_{1,1} \omega,
\end{aligned}
$$

with $c_{1}=T c$. With $c_{1}$ defined in this way, $h_{21,1}$ can be computed as the solution of

$$
\left\{\begin{aligned}
\dot{h}_{21,1}(t)-T A(t) h_{21,1}(t)+i \omega T h_{21,1}(t)-T C\left(t ; v_{1}(t), v_{1}(t), \bar{v}_{1}(t)\right) & \\
-2 T B\left(t ; v_{1}(t), h_{11,1}(t)\right)-T B\left(t ; h_{20,1}(t), \bar{v}_{1}(t)\right)+2 i c_{1} T v_{1}(t) & \\
+2 \alpha_{1,1} T\left(A(t) v_{1}(t)-i \omega v_{1}(t)\right) & =0, t \in[0,1], \\
h_{21,1}(1)-h_{21,1}(0) & =0 \\
\int_{0}^{1}\left\langle v_{1}^{*}(t), h_{21,1}(t)\right\rangle d t & =0
\end{aligned}\right.
$$

where $h_{21}(\tau)=h_{21,1}(\tau / T) /(\sqrt{T} T)$.

Next, the rescaling of the BVP for $h_{30}(\tau)$ gives

$$
\left\{\begin{aligned}
\dot{h}_{30,1}(t)-T A(t) h_{30,1}(t)+3 i \omega T h_{30,1}(t) & \\
-T C\left(t ; v_{1}(t), v_{1}(t), v_{1}(t)\right)-3 T B\left(t ; v_{1}(t), h_{20,1}(t)\right) & =0, t \in[0,1], \\
h_{30,1}(1)-h_{30,1}(0) & =0,
\end{aligned}\right.
$$

with $h_{30}(\tau)=h_{30,1}(\tau / T) /(\sqrt{T} T)$.

Now, we come to the fourth order terms where the rescaled $h_{31,1}(t)$ is the solution of (4.19)

$$
\left\{\begin{array}{r}
\dot{h}_{31,1}(t)-T A(t) h_{31,1}(t)+2 i \omega T h_{31,1}(t)-T D\left(t ; v_{1}(t), v_{1}(t), v_{1}(t), \bar{v}_{1}(t)\right) \\
-3 T C\left(t ; v_{1}(t), v_{1}(t), h_{11,1}(t)\right)-3 T C\left(t ; v_{1}(t), \bar{v}_{1}(t), h_{20,1}(t)\right) \\
-3 T B\left(t ; h_{11,1}(t), h_{20,1}(t)\right)-3 T B\left(t ; v_{1}(t), h_{21,1}(t)\right)-T B\left(t ; \bar{v}_{1}(t), h_{30,1}(t)\right) \\
+6 i c_{1} T h_{20,1}(t)+3 \alpha_{1,1} T\left(A(t) h_{20,1}(t)-2 i \omega h_{20,1}(t)+B\left(t ; v_{1}(t), v_{1}(t)\right)\right)=0, t \in[0,1], \\
h_{31,1}(1)-h_{31,1}(0)=0,
\end{array}\right.
$$

where $h_{31}(\tau)=h_{31,1}(\tau / T) / T^{2}$, and the rescaled $h_{22,1}(t)$ of

$$
\left\{\begin{aligned}
\dot{h}_{22,1}(t)-T A(t) h_{22,1}(t)-T D\left(t ; v_{1}(t), v_{1}(t), \bar{v}_{1}(t), \bar{v}_{1}(t)\right) & \\
-T C\left(t ; v_{1}(t), v_{1}(t), h_{02,1}(t)\right)-4 T C\left(t ; v_{1}(t), \bar{v}_{1}(t), h_{11,1}(t)\right) & \\
-T C\left(t ; \bar{v}_{1}(t), \bar{v}_{1}(t), h_{20,1}(t)\right)-2 T B\left(t ; h_{11,1}(t), h_{11,1}(t)\right) & \\
-2 T B\left(t ; v_{1}(t), h_{12,1}(t)\right)-T B\left(t ; h_{02,1}(t), h_{20,1}(t)\right) & \\
-2 T B\left(t ; \bar{v}_{1}(t), h_{21,1}(t)\right)+4 \alpha_{1,1} T\left(A(t) h_{11,1}(t)\right. & \\
\left.+B\left(t ; v_{1}(t), \bar{v}_{1}(t)\right)-\alpha_{1,1} F\left(u_{1}(t)\right)\right)+4 \alpha_{2,1} T F\left(u_{1}(t)\right) & =0, t \in[0,1], \\
h_{22,1}(1)-h_{22,1}(0) & =0, \\
\int_{0}^{1}\left\langle\varphi_{1}^{*}(t), h_{22,1}(t)\right\rangle d t & =0,
\end{aligned}\right.
$$


where $h_{22}(\tau)=h_{22,1}(\tau / T) / T^{2}$, and

$$
\begin{aligned}
\alpha_{2,1}= & \frac{1}{4} \int_{0}^{1}\left\langle\varphi_{1}^{*}(t), D\left(t ; v_{1}(t), v_{1}(t), \bar{v}_{1}(t), \bar{v}_{1}(t)\right)+C\left(t ; v_{1}(t), v_{1}(t), h_{02,1}(t)\right)\right. \\
& +4 C\left(t ; v_{1}(t), \bar{v}_{1}(t), h_{11,1}(t)\right)+C\left(t ; \bar{v}_{1}(t), \bar{v}_{1}(t), h_{20,1}(t)\right) \\
& +2 B\left(t ; h_{11,1}(t), h_{11,1}(t)\right)+2 B\left(t ; v_{1}(t), h_{12,1}(t)\right)+B\left(t ; h_{02,1}(t), h_{20,1}(t)\right) \\
& \left.+2 B\left(t ; \bar{v}_{1}(t), h_{21,1}(t)\right)-4 \alpha_{1,1}\left(A(t) h_{11,1}(t)+B\left(t ; v_{1}(t), \bar{v}_{1}(t)\right)\right)\right\rangle d t+\alpha_{1,1}^{2},
\end{aligned}
$$

with $\alpha_{2,1}=T^{2} \alpha_{2}$.

At last, the critical coefficient $e$ is determined by

$$
\begin{aligned}
e= & \frac{1}{12 T^{2}} \int_{0}^{1}\left\langle v_{1}^{*}(t), E\left(t ; v_{1}(t), v_{1}(t), v_{1}(t), \bar{v}_{1}(t), \bar{v}_{1}(t)\right)+D\left(t ; v_{1}(t), v_{1}(t), v_{1}(t), h_{02,1}(t)\right)\right. \\
& +6 D\left(t ; v_{1}(t), v_{1}(t), \bar{v}_{1}(t), h_{11,1}(t)\right)+3 D\left(t ; v_{1}(t), \bar{v}_{1}(t), \bar{v}_{1}(t), h_{20,1}(t)\right) \\
& +6 C\left(t ; v_{1}(t), h_{11,1}(t), h_{11,1}(t)\right)+3 C\left(t ; v_{1}(t), v_{1}(t), h_{12,1}(t)\right) \\
& +3 C\left(t ; v_{1}(t), h_{02,1}(t), h_{20,1}(t)\right)+6 C\left(t ; \bar{v}_{1}(t), h_{11,1}(t), h_{20,1}(t)\right) \\
& +6 C\left(t ; v_{1}(t), \bar{v}_{1}(t), h_{21,1}(t)\right)+C\left(t ; \bar{v}_{1}(t), \bar{v}_{1}(t), h_{30,1}(t)\right) \\
& +3 B\left(t ; h_{12,1}(t), h_{20,1}(t)\right)+6 B\left(t ; h_{11,1}(t), h_{21,1}(t)\right)+3 B\left(t ; v_{1}(t), h_{22,1}(t)\right) \\
& +B\left(t ; h_{02,1}(t), h_{30,1}(t)\right)+2 B\left(t ; \bar{v}_{1}(t), h_{31,1}(t)\right)-12 \alpha_{2,1} A(t) v_{1}(t) \\
& -6 \alpha_{1,1}\left(A(t) h_{21,1}(t)+2 B\left(t ; v_{1}(t), h_{11,1}(t)\right)+C\left(t ; v_{1}(t), v_{1}(t), \bar{v}_{1}(t)\right)\right. \\
& \left.\left.+B\left(t ; h_{20,1}(t), \bar{v}_{1}(t)\right)-2 \alpha_{1,1} A(t) v_{1}(t)\right)\right\rangle d t+\alpha_{2,1} i \frac{\omega}{T^{2}}+\alpha_{1,1} i \frac{c_{1}}{T^{2}}-\alpha_{1,1}^{2} i \frac{\omega}{T^{2}}
\end{aligned}
$$

We now compute the vector approximations for the previously defined functions. We compute $v_{1 M}$ by solving the discretization of (4.17)

$$
\left[\begin{array}{cc}
(D-T A(t)+i \omega T L)_{C \times M} & p_{2} \\
\delta_{0}-\delta_{1} & 0
\end{array}\right]\left[\begin{array}{c}
v_{1,1 M} \\
a
\end{array}\right]=\left[\begin{array}{c}
0_{C \times 1} \\
0_{n \times 1} \\
1
\end{array}\right],
$$

with $a=0$, and where $q_{2}$ is the normalized right null-vector of the complex matrix $K=$ $\left[\begin{array}{c}(D-T A(t)+i \omega T L)_{C \times M} \\ \delta_{0}-\delta_{1}\end{array}\right]$ and $p_{2}$ the normalized right null-vector of $K^{\mathrm{H}}$. This vector is then rescaled to ensure that $\sum_{i=0}^{N-1} \sum_{j=0}^{m} \sigma_{j}\left\langle\left(v_{1 M}\right)_{i, j},\left(v_{1 M}\right)_{i, j}\right\rangle=1$.

$\varphi_{1, W_{1}}^{*}$ is computed as in section 4.3. For the computation of $v_{1}^{*}$ we apply Proposition B.2 from the supplementary files, which makes it possible to compute $v_{1}^{*}$ by solving

$$
\left[\left(v_{1}^{*}\right)_{W}^{\mathrm{H}} \quad a\right]\left[\begin{array}{cc}
(D-T A(t)+i \omega T L)_{C \times M} & p_{2} \\
\delta_{0}-\delta_{1} & 0
\end{array}\right]=\left[\begin{array}{cc}
0_{M \times 1} & 1
\end{array}\right] .
$$

We then approximate $I=\int_{0}^{1}\left\langle v_{1}^{*}(t), v_{1}(t)\right\rangle d t$ by $I_{1}=\left(v_{1}^{*}\right)_{W_{1}}^{\mathrm{H}} L_{C \times M} v_{1 M}$ and rescale $v_{1 W}^{*}$ so that $I_{1}=1$.

The second order terms are approximated by

$$
\left[\begin{array}{c}
(D-T A(t)+2 i \omega T L)_{C \times M} \\
\delta_{0}-\delta_{1}
\end{array}\right] h_{20,1 M}=\left[\begin{array}{c}
T B_{C \times M \times M} v_{1 M} v_{1 M} \\
0_{n \times 1}
\end{array}\right]
$$


and

$$
\left[\begin{array}{cc}
(D-T A(t))_{C \times M} & p \\
\delta_{0}-\delta_{1} & 0
\end{array}\right]\left[\begin{array}{c}
h_{11,1 M} \\
a
\end{array}\right]=\left[\begin{array}{c}
T B_{C \times M \times M} v_{1 M} \bar{v}_{1 M}-\alpha_{1,1} T g_{C} \\
0_{n \times 1} \\
0
\end{array}\right]
$$

with $\alpha_{1,1}$ computed as

$$
\alpha_{1,1}=\left(\varphi_{1}^{*}\right)_{W_{1}}^{\mathrm{T}} B_{C \times M \times M} v_{1 M} \bar{v}_{1 M} .
$$

An approximation to the rescaled normal form coefficient $c_{1}$ is given by

$$
\begin{aligned}
c_{1}= & -\frac{i}{2}\left(v_{1}^{*}\right)_{W_{1}}^{\mathrm{H}}\left(C_{C \times M \times M \times M} v_{1 M} v_{1 M} \bar{v}_{1 M}+2 B_{C \times M \times M} v_{1 M} h_{11,1 M}\right. \\
& \left.+B_{C \times M \times M} \bar{v}_{1 M} h_{20,1 M}-2 \alpha_{1,1} A_{C \times M} v_{1 M}\right)+\alpha_{1,1} \omega,
\end{aligned}
$$

where in MATCONT an extra check is done to ensure that this coefficient is indeed purely imaginary.

Next, we determine the third order coefficients of the center manifold expansion, namely,

$$
\left[\begin{array}{cc}
(D-T A(t)+i \omega T L)_{C \times M} & p_{2} \\
\delta_{0}-\delta_{1} & 0
\end{array}\right]\left[\begin{array}{c}
h_{21,1 M} \\
a
\end{array}\right]=\left[\begin{array}{c}
\mathrm{R} \\
0_{n \times 1} \\
0
\end{array}\right],
$$

where

$$
\begin{aligned}
\mathrm{R}= & T C_{C \times M \times M \times M} v_{1 M} v_{1 M} \bar{v}_{1 M}+2 T B_{C \times M \times M} v_{1 M} h_{11,1 M}+T B_{C \times M \times M} h_{20,1 M} \bar{v}_{1 M} \\
& -2 i c_{1} T L_{C \times M} v_{1 M}-2 \alpha_{1,1} T\left(A_{C \times M} v_{1 M}-i \omega L_{C \times M} v_{1 M}\right)
\end{aligned}
$$

and $a=0$, and

$$
\left[\begin{array}{c}
(D-T A(t)+3 i \omega T L)_{C \times M} \\
\delta_{0}-\delta_{1}
\end{array}\right] h_{30,1 M}=\left[\begin{array}{c}
\mathrm{R} \\
0_{n \times 1}
\end{array}\right],
$$

with $\mathrm{R}=T C_{C \times M \times M \times M} v_{1 M} v_{1 M} v_{1 M}+3 T B_{C \times M \times M} v_{1 M} h_{20,1 M}$.

The approximation to (4.19) is given by

$$
\left[\begin{array}{c}
(D-T A(t)+2 i \omega T L)_{C \times M} \\
\delta_{0}-\delta_{1}
\end{array}\right] h_{31,1 M}=\left[\begin{array}{c}
\mathrm{R} \\
0_{n \times 1}
\end{array}\right],
$$

with

$$
\begin{aligned}
\mathrm{R}= & T D_{C \times M \times M \times M \times M} v_{1 M} v_{1 M} v_{1 M} \bar{v}_{1 M}+3 T C_{C \times M \times M \times M} v_{1 M} v_{1 M} h_{11,1 M} \\
& +3 T C_{C \times M \times M \times M} v_{1 M} \bar{v}_{1 M} h_{20,1 M}+3 T B_{C \times M \times M} h_{11,1 M} h_{20,1 M} \\
& +3 T B_{C \times M \times M} v_{1 M} h_{21,1 M}+T B_{C \times M \times M} \bar{v}_{1 M} h_{30,1 M}-6 i c_{1} T L_{C \times M} h_{20,1 M} \\
& -3 \alpha_{1,1} T\left(A_{C \times M} h_{20,1 M}-2 i \omega L_{C \times M} h_{20,1 M}+B_{C \times M \times M} v_{1 M} v_{1 M}\right)
\end{aligned}
$$

while

$$
\begin{aligned}
\alpha_{2,1}= & \frac{1}{4}\left(\varphi_{1}^{*}\right)_{W_{1}}^{\mathrm{T}}\left(D_{C \times M \times M \times M \times M} v_{1 M} v_{1 M} \bar{v}_{1 M} \bar{v}_{1 M}+C_{C \times M \times M \times M} v_{1 M} v_{1 M} h_{02,1 M}\right. \\
& +4 C_{C \times M \times M \times M} v_{1 M} \bar{v}_{1 M} h_{11,1 M}+C_{C \times M \times M \times M} \bar{v}_{1 M} \bar{v}_{1 M} h_{20,1 M} \\
& +2 B_{C \times M \times M} h_{11,1 M} h_{11,1 M}+2 B_{C \times M \times M} v_{1 M} h_{12,1 M}+B_{C \times M \times M} h_{02,1 M} h_{20,1 M} \\
& \left.+2 B_{C \times M \times M} \bar{v}_{1 M} h_{21,1 M}-4 \alpha_{1,1}\left(A_{C \times M} h_{11,1 M}+B_{C \times M \times M} v_{1 M} \bar{v}_{1 M}\right)\right)+\alpha_{1,1}^{2} .
\end{aligned}
$$


The last fourth order term needed is given by

$$
\left[\begin{array}{cc}
(D-T A(t))_{C \times M} & p \\
\delta_{0}-\delta_{1} & \\
\left(\varphi_{1}^{*}\right)_{W_{1}}^{\mathrm{T}} L_{C \times M} & 0
\end{array}\right]\left[\begin{array}{c}
h_{22,1 M} \\
a
\end{array}\right]=\left[\begin{array}{c}
\mathrm{R} \\
0_{n \times 1} \\
0
\end{array}\right],
$$

with

$$
\begin{aligned}
\mathrm{R}= & T D_{C \times M \times M \times M \times M} v_{1 M} v_{1 M} \bar{v}_{1 M} \bar{v}_{1 M}+T C_{C \times M \times M \times M} v_{1 M} v_{1 M} h_{02,1 M} \\
& +4 T C_{C \times M \times M \times M} v_{1 M} \bar{v}_{1 M} h_{11,1 M}+T C_{C \times M \times M \times M} \bar{v}_{1 M} \bar{v}_{1 M} h_{20,1 M} \\
& +2 T B_{C \times M \times M} h_{11,1 M} h_{11,1 M}+2 T B_{C \times M \times M} v_{1 M} h_{12,1 M}+T B_{C \times M \times M} h_{02,1 M} h_{20,1 M} \\
& +2 T B_{C \times M \times M} \bar{v}_{1 M} h_{21,1 M}-4 \alpha_{1,1} T\left(A_{C \times M} h_{11,1 M}+B_{C \times M \times M} v_{1 M} \bar{v}_{1 M}\right. \\
& \left.-\alpha_{1} g_{C}\right)-4 \alpha_{2,1} T g_{C}
\end{aligned}
$$

and $a=0$. Now, we have all the information needed to compute the fifth order coefficient of the normal form, namely,

$$
\begin{aligned}
e= & \frac{1}{12 T^{2}}\left(v_{1}^{*}\right)_{W_{1}}^{\mathrm{H}}\left(E_{C \times M \times M \times M \times M \times M} v_{1 M} v_{1 M} v_{1 M} \bar{v}_{1 M} \bar{v}_{1 M}\right. \\
& +D_{C \times M \times M \times M \times M} v_{1 M} v_{1 M} v_{1 M} h_{02,1 M}+6 D_{C \times M \times M \times M \times M} v_{1 M} v_{1 M} \bar{v}_{1 M} h_{11,1 M} \\
& +3 D_{C \times M \times M \times M \times M} v_{1 M} \bar{v}_{1 M} \bar{v}_{1 M} h_{20,1 M}+6 C_{C \times M \times M \times M} v_{1 M} h_{11,1 M} h_{11,1 M} \\
& +3 C_{C \times M \times M \times M} v_{1 M} v_{1 M} h_{12,1 M}+3 C_{C \times M \times M \times M} v_{1 M} h_{02,1 M} h_{20,1 M} \\
& +6 C_{C \times M \times M \times M} \bar{v}_{1 M} h_{11,1 M} h_{20,1 M}+6 C_{C \times M \times M \times M} v_{1 M} \bar{v}_{1 M} h_{21,1 M} \\
& +C_{C \times M \times M \times M} \bar{v}_{1 M} \bar{v}_{1 M} h_{30,1 M}+3 B_{C \times M \times M} h_{12,1 M} h_{20,1 M} \\
& +6 B_{C \times M \times M} h_{11,1 M} h_{21,1 M}+3 B_{C \times M \times M} v_{1 M} h_{22,1 M} \\
& +B_{C \times M \times M} h_{02,1 M} h_{30,1 M}+2 B_{C \times M \times M} \bar{v}_{1 M} h_{31,1 M}-12 \alpha_{2,1} A_{C \times M} v_{1 M} \\
& -6 \alpha_{1,1}\left(A_{C \times M} h_{21,1 M}+2 B_{C \times M \times M} v_{1 M} h_{11,1 M}+C_{C \times M \times M \times M} v_{1 M} v_{1 M} \bar{v}_{1 M}\right. \\
& \left.\left.+B_{C \times M \times M} h_{20,1 M} \bar{v}_{1 M}-2 \alpha_{1,1} A_{C \times M} v_{1 M}\right)\right)+\alpha_{2,1} i \frac{\omega}{T^{2}}+\alpha_{1,1} i \frac{c_{1}}{T^{2}}-\alpha_{1,1}^{2} i \frac{\omega}{T^{2}}
\end{aligned}
$$

4.5. Strong resonance 1:1 bifurcation. In addition to the rescaled generalized eigenfunction $v_{1,1}$ defined by (4.3), the second rescaled generalized eigenfunction associated to multiplier 1 can be found by solving

$$
\left\{\begin{aligned}
\dot{v}_{2,1}(t)-T A(t) v_{2,1}(t)+T v_{1,1}(t) & =0, \quad t \in[0,1] \\
v_{2,1}(1)-v_{2,1}(0) & =0 \\
\int_{0}^{1}\left\langle v_{2,1}(t), F\left(u_{1}(t)\right)\right\rangle d t & =0
\end{aligned}\right.
$$

with $v_{2}(\tau)=v_{2,1}(\tau / T)$.

The adjoint eigenfunction $\varphi_{1}^{*}$ is determined by the first two equations of (4.4) and the normalization condition

$$
\int_{0}^{1}\left\langle\varphi_{1}^{*}(t), v_{2,1}(t)\right\rangle d t-1=0 .
$$


We also need the first rescaled generalized adjoint eigenfunction that is given by

$$
\left\{\begin{aligned}
\dot{v}_{1,1}^{*}(t)+T A^{\mathrm{T}}(t) v_{1,1}^{*}(t)-T \varphi_{1}^{*}(t) & =0, \quad t \in[0,1], \\
v_{1,1}^{*}(1)-v_{1,1}^{*}(0) & =0 \\
\int_{0}^{1}\left\langle v_{1,1}^{*}(t), v_{2,1}(t)\right\rangle d t & =0
\end{aligned}\right.
$$

with $v_{1}^{*}(\tau)=v_{1,1}^{*}(\tau / T) / T$. Now, we have all the information needed to compute the two critical coefficients, namely,

$$
a=\frac{1}{2} \int_{0}^{1}\left\langle\varphi_{1}^{*}(t), 2 A(t) v_{1,1}(t)+B\left(t ; v_{1,1}(t), v_{1,1}(t)\right)\right\rangle d t
$$

and

$$
\begin{aligned}
b=\int_{0}^{1}\langle & \left.\varphi_{1}^{*}(t), B\left(t ; v_{1,1}(t), v_{2,1}(t)\right)+A(t) v_{2,1}(t)\right\rangle d t \\
& +\int_{0}^{1}\left\langle v_{1,1}^{*}(t), 2 A(t) v_{1,1}(t)+B\left(t ; v_{1,1}(t), v_{1,1}(t)\right)\right\rangle d t .
\end{aligned}
$$

The implementation in MATCONT is straightforward and relies on earlier explained techniques, so we will omit further details.

4.6. Strong resonance 1:2 bifurcation. The rescaled eigenfunction associated to multiplier -1 is given by (4.10), and the rescaled generalized eigenfunction is the solution of

$$
\left\{\begin{aligned}
\dot{v}_{2,1}(t)-T A(t) v_{2,1}(t)+T v_{1,1}(t) & =0, t \in[0,1] \\
v_{2,1}(1)+v_{2,1}(0) & =0 \\
\int_{0}^{1}\left\langle v_{2,1}(t), v_{1,1}(t)\right\rangle d t & =0
\end{aligned}\right.
$$

where $v_{2}(\tau)=v_{2,1}(\tau / T) / \sqrt{T}$.

The rescaled adjoint eigenfunctions are determined by (4.11), (4.12) but with normalization condition $\int_{0}^{1}\left\langle v_{1,1}^{*}(t), v_{2,1}(t)\right\rangle d t=1$ and

$$
\left\{\begin{aligned}
\dot{v}_{2,1}^{*}(t)+T A^{\mathrm{T}}(t) v_{2,1}^{*}(t)-T v_{1,1}^{*}(t) & =0, \quad t \in[0,1] \\
v_{2,1}^{*}(1)+v_{2,1}^{*}(0) & =0 \\
\int_{0}^{1}\left\langle v_{2,1}(t), v_{2,1}^{*}(t)\right\rangle d t & =0
\end{aligned}\right.
$$

where $v_{2}^{*}(\tau)=v_{2,1}^{*}(\tau / T) / \sqrt{T}$.

With $\alpha_{1}$ defined as

$$
\alpha_{1}=\frac{1}{2} \int_{0}^{1}\left\langle\varphi_{1}^{*}(t), B\left(t ; v_{1,1}(t), v_{1,1}(t)\right)\right\rangle d t
$$

with $\alpha_{1}=T \alpha$, let $h_{20,1}(t)$ be the unique solution of the rescaled BVP

$$
\left\{\begin{aligned}
\dot{h}_{20,1}(t)-T A(t) h_{20,1}(t)-T B\left(t ; v_{1,1}(t), v_{1,1}(t)\right)+2 \alpha_{1} T F\left(u_{1}(t)\right) & =0, \quad t \in[0,1] \\
h_{20,1}(1)-h_{20,1}(0) & =0 \\
\int_{0}^{1}\left\langle\varphi_{1}^{*}(t), h_{20,1}(t)\right\rangle d t-\int_{0}^{1}\left\langle\varphi_{1}^{*}(t), B\left(v_{1,1}(t), v_{2,1}(t)\right)\right\rangle d t & =0
\end{aligned}\right.
$$


where $h_{20}(\tau)=h_{20,1}(\tau / T) / T$.

With $h_{11,1}(t)$ being the rescaling of the function $h_{11}(\tau)$, the solution of

$$
\left\{\begin{aligned}
\dot{h}_{11,1}(t)-T A(t) h_{11,1}(t)-T B\left(t ; v_{1,1}(t), v_{2,1}(t)\right)+T h_{20,1}(t) & =0, t \in[0,1] \\
h_{11,1}(1)-h_{11,1}(0) & =0 \\
\int_{0}^{1}\left\langle\varphi_{1}^{*}(t), h_{11,1}(t)\right\rangle d t-\frac{1}{2} \int_{0}^{1}\left\langle\varphi_{1}^{*}(t), B\left(v_{2,1}(t), v_{2,1}(t)\right)\right\rangle d t & =0
\end{aligned}\right.
$$

where $h_{11}(\tau)=h_{11,1}(\tau / T) / T$, we are able to obtain the two normal form coefficients by the expressions

$$
a_{1}=\frac{1}{6} \int_{0}^{1}\left\langle v_{1,1}^{*}(t), C\left(t ; v_{1,1}(t), v_{1,1}(t), v_{1,1}(t)\right)+3 B\left(t ; v_{1,1}(t), h_{20,1}(t)\right)-6 \alpha_{1} A(t) v_{1,1}(t)\right\rangle d t,
$$

with $a_{1}=T a$, and

$$
\begin{aligned}
b=\frac{1}{2 T} \int_{0}^{1}\left\langle v_{1,1}^{*}(t),-\right. & 2 \alpha_{1} A(t) v_{2,1}(t)+C\left(t ; v_{1,1}(t), v_{1,1}(t), v_{2,1}(t)\right) \\
& \left.+B\left(t ; h_{20,1}(t), v_{2,1}(t)\right)+2 B\left(t ; h_{11,1}(t), v_{1,1}(t)\right)\right\rangle d t \\
+ & \frac{1}{2 T} \int_{0}^{1}\left\langle v_{2,1}^{*}(t), C\left(t ; v_{1,1}(t), v_{1,1}(t), v_{1,1}(t)\right)+3 B\left(t ; v_{1,1}(t), h_{20,1}(t)\right)-6 \alpha_{1} A(t) v_{1,1}(t)\right\rangle d t .
\end{aligned}
$$

For the implementation details we will just highlight the differences from the previous cases. Formula (4.13) gives us the value of $v_{1,1}$ in the mesh points. However, since $v_{1,1}$ is used in the integral condition for $v_{2,1}$, we have to transfer this vector to the collocation points and multiply it with the Gauss-Legendre weights and the lengths of the corresponding intervals to obtain $\left(v_{1,1}\right)_{W_{1}}$. The computation of $v_{2,1}$ is then straightforward.

Using the antiperiodic version of Proposition B.3, we approximate the adjoint generalized eigenfunction by solving

$$
\left[\left(v_{2,1}^{*}\right)_{W}^{\mathrm{T}} \quad a\right]\left[\begin{array}{cc}
(D-T A(t))_{C \times M} & v_{2,1 C} \\
\delta_{0}+\delta_{1} & 0_{n \times 1} \\
q_{1}^{\mathrm{T}} & 0
\end{array}\right]=\left[\begin{array}{ll}
-T\left(v_{1,1}^{*}\right)_{W_{1}}^{\mathrm{T}} L_{C \times M} & 0
\end{array}\right] .
$$

Now, $h_{20,1 M}$ is found by discretizing (4.21), i.e.,

$$
\left[\begin{array}{cc}
(D-T A(t))_{C \times M} & p \\
\delta_{0}-\delta_{1} & 0 \\
\left(\varphi_{1}^{*}\right)_{W_{1}}^{\mathrm{T}} L_{C \times M} & 0
\end{array}\right]\left[\begin{array}{c}
h_{20,1 M} \\
a
\end{array}\right]=\left[\begin{array}{c}
T B_{C \times M \times M} v_{1,1 M} v_{1,1 M}-2 \alpha_{1} T g_{C} \\
0_{n \times 1} \\
\left(\varphi_{1}^{*}\right)_{W_{1}}^{\mathrm{T}} B_{C \times M \times M} v_{1,1 M} v_{2,1 M}
\end{array}\right] .
$$

4.7. Strong resonance 1:3 bifurcation. The BVPs for the rescaled eigenfunction and its adjoint belonging to eigenvalue $e^{i \frac{2 \pi}{3}}$ are determined by

$$
\left\{\begin{aligned}
\dot{v}_{1}(t)-T A(t) v_{1}(t) & =0, t \in[0,1] \\
v_{1}(1)-e^{i \frac{2 \pi}{3}} v_{1}(0) & =0 \\
\int_{0}^{1}\left\langle v_{1}(t), v_{1}(t)\right\rangle d t-1 & =0
\end{aligned}\right.
$$


with $v(\tau)=v_{1}(\tau / T) / \sqrt{T}$, and

$$
\left\{\begin{aligned}
\dot{v}_{1}^{*}(t)+T A^{\mathrm{T}}(t) v_{1}^{*}(t) & =0, t \in[0,1] \\
v_{1}^{*}(1)-e^{i \frac{2 \pi}{3}} v_{1}^{*}(0) & =0 \\
\int_{0}^{1}\left\langle v_{1}^{*}(t), v_{1}(t)\right\rangle d t-1 & =0
\end{aligned}\right.
$$

where $v^{*}(\tau)=v_{1}^{*}(\tau / T) / \sqrt{T}$. The adjoint eigenfunction corresponding to the trivial multiplier is given by (4.11).

These eigenfunctions already make it possible to compute the rescaled normal form coefficients

$$
\alpha_{1,1}=\int_{0}^{1}\left\langle\varphi_{1}^{*}(t), B\left(v_{1}(t), \bar{v}_{1}(t)\right)\right\rangle d t
$$

where $\alpha_{1,1}=T \alpha_{1}$, and

$$
b_{1}=\frac{1}{2} \int_{0}^{1}\left\langle v_{1}^{*}(t), B\left(\bar{v}_{1}(t), \bar{v}_{1}(t)\right)\right\rangle d t
$$

with $b_{1}=\sqrt{T} b$.

The rescaled second order functions in the center manifold expansion are solutions of

$$
\left\{\begin{aligned}
\dot{h}_{20,1}(t)-T A(t) h_{20,1}(t)-T B\left(v_{1}(t), v_{1}(t)\right)+2 \bar{b}_{1} T \bar{v}_{1}(t) & =0, t \in[0, T], \\
h_{20,1}(1)-e^{i \frac{4 \pi}{3}} h_{20,1}(0) & =0, \\
\int_{0}^{1}\left\langle\bar{v}_{1}^{*}(t), h_{20,1}(t)\right\rangle d t & =0,
\end{aligned}\right.
$$

with $h_{20}(\tau)=h_{20,1}(\tau / T) / T$, and

$$
\left\{\begin{aligned}
\dot{h}_{11,1}(t)-T A(t) h_{11,1}-T B\left(v_{1}(t), \bar{v}_{1}(t)\right)+\alpha_{1,1} T F\left(u_{1}(t)\right) & =0, \quad t \in[0,1], \\
h_{11,1}(1)-h_{11,1}(0) & =0 \\
\int_{0}^{1}\left\langle\varphi_{1}^{*}(t), h_{11,1}(t)\right\rangle d t & =0
\end{aligned}\right.
$$

with $h_{11}(\tau)=h_{11,1}(\tau / T) / T$. This all results then in

$$
\begin{aligned}
c= & \frac{1}{2 T} \int_{0}^{1}\left\langle v_{1}^{*}(t), C\left(v_{1}(t), v_{1}(t), \bar{v}_{1}(t)\right)+2 B\left(v_{1}(t), h_{11,1}(t)\right)\right. \\
& \left.+B\left(\bar{v}_{1}(t), h_{20,1}(t)\right)-2 \alpha_{1,1} A v_{1}(t)\right\rangle d t .
\end{aligned}
$$

We now come to the implementation details in MATCONT. We again highlight only the differences from the implementation details given in the previous sections. Eigenfunction $v_{1}$, determined by (4.23), is computed by

$$
\left[\begin{array}{cc}
(D-T A(t))_{C \times M} & p_{3} \\
\delta_{0}-e^{-i \frac{2 \pi}{3}} \delta_{1} & 0
\end{array}\right]\left[\begin{array}{c}
v_{1 M} \\
a
\end{array}\right]=\left[\begin{array}{c}
0_{C \times 1} \\
0_{n \times 1} \\
1
\end{array}\right] .
$$

We normalize $v_{1 M}$ by requiring $\sum_{i=0}^{N-1} \sum_{j=0}^{m} \sigma_{j}\left\langle\left(v_{1 M}\right)_{i, j},\left(v_{1 M}\right)_{i, j}\right\rangle=1$, where $\sigma_{j}$ is the Lagrange quadrature coefficient. $q_{3}$ is the normalized right null-vector of $K=\left[\begin{array}{c}(D-T A(t))_{C \times M} \\ \delta_{0}-e^{-i \frac{2 \pi}{3}} \delta_{1}\end{array}\right]$, and $p_{3}$ is the normalized right null-vector of $K^{\mathrm{H}}$. 
To compute the adjoint eigenfunction $v_{1}^{*}$, we apply Proposition B.1 from the supplementary files but with boundary condition $\zeta(0)-e^{-i \frac{2 \pi}{3}} \zeta(1)$ and obtain

$$
\left[\left(v_{1}^{*}\right)_{W}^{\mathrm{H}} \quad a\right]\left[\begin{array}{cc}
(D-T A(t))_{C \times M} & p_{3} \\
\delta_{0}-e^{-i \frac{2 \pi}{3}} \delta_{1} & 0 \\
q_{3}^{\mathrm{H}} & 0
\end{array}\right]=\left[\begin{array}{ll}
0_{M \times 1} & 1
\end{array}\right] .
$$

$v_{1 W}^{*}$ is rescaled such that $\left(v_{1}^{*}\right)_{W_{1}}^{\mathrm{H}} L_{C \times M} v_{1 M}=1$.

By first computing the complex conjugate of $h_{20,1}$ we can use the same matrix as in (4.26), except for the last line which represents the integral condition, to get

$$
\left[\begin{array}{cc}
(D-T A(t))_{C \times M} & p_{3} \\
\delta_{0}-e^{-i \frac{2 \pi}{3}} \delta_{1} & \\
\left(v_{1}^{*}\right)_{W_{1}}^{\mathrm{H}} L_{C \times M} & 0
\end{array}\right]\left[\begin{array}{c}
\bar{h}_{20,1 M} \\
a
\end{array}\right]=\left[\begin{array}{c}
T B_{C \times M \times M} \bar{v}_{1 M} \bar{v}_{1 M}-2 b_{1} T v_{1 C} \\
0_{n \times 1} \\
0
\end{array}\right] .
$$

4.8. Strong resonance 1:4 bifurcation. The rescaled eigenfunction and the adjoint eigenfunction corresponding to multiplier $e^{i \frac{\pi}{2}}$ are given by the solution of

$$
\left\{\begin{aligned}
\dot{v}_{1}(t)-T A(t) v_{1}(t) & =0, t \in[0,1] \\
v_{1}(1)-e^{i \frac{\pi}{2}} v_{1}(0) & =0 \\
\int_{0}^{1}\left\langle v_{1}(t), v_{1}(t)\right\rangle d t-1 & =0
\end{aligned}\right.
$$

with $v(\tau)=v_{1}(\tau / T) / \sqrt{T}$, and

$$
\left\{\begin{aligned}
\dot{v}_{1}^{*}(t)+T A^{\mathrm{T}}(t) v_{1}^{*}(t) & =0, t \in[0,1] \\
v_{1}^{*}(1)-e^{i \frac{\pi}{2}} v_{1}^{*}(0) & =0 \\
\int_{0}^{1}\left\langle v_{1}^{*}(t), v_{1}(t)\right\rangle d t-1 & =0
\end{aligned}\right.
$$

where $v^{*}(\tau)=v_{1}^{*}(\tau / T) / \sqrt{T}$, respectively. We also need the functions $\varphi_{1}^{*}(t)$ and $h_{11,1}(t)$ defined by (4.11) and (4.25) and the value of $\alpha_{1,1}$ given by (4.24).

The other second order coefficient of the center manifold is determined by

$$
\left\{\begin{aligned}
\dot{h}_{20,1}(t)-T A(t) h_{20,1}(t)-T B\left(v_{1}(t), v_{1}(t)\right) & =0, t \in[0,1] \\
h_{20,1}(1)+h_{20,1}(0) & =0
\end{aligned}\right.
$$

with $h_{20}(\tau)=h_{20,1}(\tau / T) / T$.

The critical normal form coefficients are then given by

$$
\begin{aligned}
\bar{c}= & \frac{1}{2 T} \int_{0}^{1}\left\langle\bar{v}_{1}^{*}(t), C\left(v_{1}(t), \bar{v}_{1}(t), \bar{v}_{1}(t)\right)+B\left(v_{1}(t), h_{02,1}(t)\right)+2 B\left(\bar{v}_{1}(t), h_{11,1}(t)\right)\right. \\
& \left.-2 \alpha_{11} A(t) \bar{v}_{1}(t)\right\rangle d t
\end{aligned}
$$

and

$$
d=\frac{1}{6 T} \int_{0}^{1}\left\langle v_{1}^{*}(t), C\left(\bar{v}_{1}(t), \bar{v}_{1}(t), \bar{v}_{1}(t)\right)+3 B\left(\bar{v}_{1}(t), h_{02,1}(t)\right)\right\rangle d t .
$$

The code is very similar to that of the strong resonance 1:3 case. 
4.9. Fold-flip bifurcation. The rescaled generalized eigenfunction $v_{1,1}$ associated to multiplier 1 is the solution of (4.3), and the eigenfunction $v_{2,1}(t)$ associated to multiplier -1 is defined by (4.10). Equation (4.4) determines the adjoint eigenfunction $\varphi_{1}^{*}(t)$ corresponding to the trivial solution, and the generalized adjoint eigenfunction $v_{1,1}^{*}(t)$ is given by (4.2). The last adjoint eigenfunction $v_{2,1}^{*}(t)$ is found by solving (4.12).

The coefficients in front of the $\xi_{1}^{2}$-terms in the corresponding normal form are given by

$$
a_{20}=\frac{1}{2} \int_{0}^{1}\left\langle\varphi_{1}^{*}(t), B\left(v_{1,1}(t), v_{1,1}(t)\right)+2 A(t) v_{1,1}(t)\right\rangle d t
$$

and $\alpha_{20}=0$.

The second order coefficients of the center manifold expansion are defined by the rescaled BVPs

$$
\left\{\begin{aligned}
\dot{h}_{20,1}(t)-T A(t) h_{20,1}(t)-T B\left(v_{1,1}(t), v_{1,1}(t)\right) & \\
+2 a_{20} T v_{1,1}(t)+2 \alpha_{20} T F\left(u_{1}(t)\right)-2 T A(t) v_{1,1}(t)-2 T F\left(u_{1}(t)\right) & =0, t \in[0,1], \\
h_{20,1}(1)-h_{20,1}(0) & =0, \\
\int_{0}^{1}\left\langle v_{1,1}^{*}(t), h_{20,1}(t)\right\rangle d t & =0,
\end{aligned}\right.
$$

with $h_{20}(\tau)=h_{20,1}(\tau / T)$,

$$
\left\{\begin{aligned}
\dot{h}_{11,1}(t)-T A(t) h_{11,1}(t)-T B\left(v_{1,1}(t), v_{2,1}(t)\right)+T b_{11} v_{2,1}(t) & \\
-T A(t) v_{2,1}(t) & =0, \quad t \in[0,1], \\
h_{11,1}(1)+h_{11,1}(0) & =0 \\
\int_{0}^{1}\left\langle v_{2,1}^{*}(t), h_{11,1}(t)\right\rangle d t & =0
\end{aligned}\right.
$$

with $h_{11}(\tau)=h_{11,1}(\tau / T) / \sqrt{T}$, and

$$
\left\{\begin{aligned}
\dot{h}_{02,1}(t)-T A(t) h_{02,1}(t)-T B\left(v_{2,1}(t), v_{2,1}(t)\right)+2 a_{02,1} T v_{1,1}(t) & \\
+2 \alpha_{02,1} T F\left(u_{1}(t)\right) & =0, \quad t \in[0,1], \\
h_{02,1}(1)-h_{02,1}(0) & =0, \\
\int_{0}^{1}\left\langle v_{1,1}^{*}(t), h_{02,1}(t)\right\rangle d t & =0,
\end{aligned}\right.
$$

with $h_{02}(\tau)=h_{02,1}(\tau / T) / T$, where

$$
\begin{gathered}
b_{11}=\int_{0}^{1}\left\langle v_{2,1}^{*}(t), B\left(v_{1,1}(t), v_{2,1}(t)\right)+A(t) v_{2,1}(t)\right\rangle d t, \\
a_{02,1}=\frac{1}{2} \int_{0}^{1}\left\langle\varphi_{1}^{*}(t), B\left(v_{2,1}(t), v_{2,1}(t)\right)\right\rangle d t
\end{gathered}
$$

with $a_{02,1}=T a_{02}$ and $\alpha_{02}=0$.

The rescaling of the last four normal form coefficients of interest gives

$$
\begin{aligned}
a_{30}=\frac{1}{6} \int_{0}^{1}\left\langle\varphi_{1}^{*}(t), C\left(v_{1,1}(t), v_{1,1}(t), v_{1,1}(t)\right)+3 B\left(h_{20,1}, v_{1,1}(t)\right)-6 a_{20} h_{20,1}(t)\right. \\
\left.+3\left(A(t) h_{20,1}(t)+B\left(v_{1,1}(t), v_{1,1}(t)\right)\right)+6\left(1-\alpha_{20}\right) A(t) v_{1,1}(t)\right\rangle d t-a_{20},
\end{aligned}
$$




$$
\begin{aligned}
b_{21}=\frac{1}{2} \int_{0}^{1}\left\langle v_{2,1}^{*}(t), C\left(v_{1,1}(t), v_{1,1}(t), v_{2,1}(t)\right)+B\left(h_{20,1}(t), v_{2,1}(t)\right)+2 B\left(h_{11,1}(t), v_{1,1}(t)\right)\right. \\
-2 a_{20} h_{11,1}(t)-2 b_{11} h_{11,1}(t)+2\left(A(t) h_{11,1}(t)+B\left(v_{1,1}(t), v_{2,1}(t)\right)\right) \\
\left.+2\left(1-\alpha_{20}\right) A(t) v_{2,1}(t)\right\rangle d t-b_{11} \\
a_{12}=\frac{1}{2 T} \int_{0}^{1}\left\langle\varphi_{1}^{*}(t), C\left(v_{1,1}(t), v_{2,1}(t), v_{2,1}(t)\right)+B\left(h_{02,1}(t), v_{1,1}(t)\right)\right. \\
\quad+2 B\left(h_{11,1}(t), v_{2,1}(t)\right)-2 b_{11} h_{02,1}(t)-2 a_{02,1} h_{20,1}(t)+A(t) h_{02,1}(t) \\
\left.+B\left(v_{2,1}(t), v_{2,1}(t)\right)-2 \alpha_{02,1} A(t) v_{1,1}(t)\right\rangle d t-\frac{a_{02,1}}{T}
\end{aligned}
$$

and

$$
\begin{aligned}
b_{03}=\frac{1}{6 T} \int_{0}^{1}\left\langle v_{2,1}^{*}(t), C\left(v_{2,1}(t), v_{2,1}(t), v_{2,1}(t)\right)+3 B\left(h_{02,1}(t), v_{2,1}(t)\right)-6 a_{02,1} h_{11,1}(t)\right. \\
\\
\left.-6 \alpha_{02,1} A(t) v_{2,1}(t)\right\rangle d t .
\end{aligned}
$$

For the computation of the needed functions and coefficients of interest, we refer the reader to the previous sections.

5. Examples. All computations in this section are performed with MATCONT [14]. In particular, the bordering methods from $[18,21]$ are used to continue the codim 1 bifurcations of limit cycles in two parameters. The algorithms described above for computing the normal form coefficients are implemented in the current version of MATCONT.

5.1. Periodic predator-prey model. Our first model is a periodically forced predator-prey system, studied in [34] using shooting techniques, and described by the differential equations

$$
\left\{\begin{array}{l}
\dot{x}=r\left(1-\frac{x}{K}\right) x-p(x, t) y, \\
\dot{y}=e p(x, t) y-d y
\end{array}\right.
$$

where $x$ and $y$ are the numbers of individuals, respectively, of prey and predator populations or suitable measures of density or biomass. The parameters present in (5.1) are the intrinsic growth rate $r$, the carrying capacity $K$, the efficiency $e$, and the death rate $d$ of the predator. The function $p(x, t)$ is the predator functional response, for which the Holling type II is chosen, with constant attack rate $a$ and half saturation $b(t)$ that varies periodically with period one (year), i.e.,

$$
p(x, t)=\frac{a x}{b(t)+x}, \quad b(t)=b_{0}(1+\varepsilon \cos (2 \pi t)) .
$$

Instead of system (5.1), we consider the extended autonomous system

$$
\left\{\begin{array}{l}
\dot{x}=r\left(1-\frac{x}{K}\right) x-\frac{a x y}{b_{0}(1+\varepsilon u)+x} \\
\dot{y}=e \frac{a x y}{b_{0}(1+\varepsilon u)+x}-d y \\
\dot{u}=u-2 \pi v-\left(u^{2}+v^{2}\right) u \\
\dot{v}=2 \pi u+v-\left(u^{2}+v^{2}\right) v
\end{array}\right.
$$


where the last two equations have a stable limit cycle with $u(t)=\cos (2 \pi t+\varphi)$ and a phase shift $\varphi$ depending on the initial conditions.

With fixed $r=2 \pi, K=e=1, a=4 \pi$, and $d=2 \pi$, we perform a bifurcation analysis with respect to the remaining parameters $\left(\varepsilon, b_{0}\right)$ obtaining the bifurcation diagram reported in Figure 1. Since the system is periodically forced, no equilibria are present. The blue curves, with labels LPC2(1) and LPC2(2), are limit point of cycle bifurcation curves of the second iterate, the magenta curves are Neimark-Sacker bifurcations (of the first or of the second iterate, respectively labeled with NS1 and NS2), and the green curves are period-doubling bifurcations, dotted when subcritical and a solid line when supercritical (with notation PD1, PD2, PD4, and PD8).

We have chosen this system as the first example since it allows us to check if the computation of the normal form coefficients $\alpha_{i}$ is correct. Indeed, in a periodically forced system the return time is independent of the distance from the limit cycle, so that the first equation in all periodic normal forms should be $\dot{\tau}=1$. For the cases GPD and $\mathrm{CH}$, as well as for the strong resonance cases R2, R3, and R4, this would imply that all $\alpha_{i}$ in the normal forms listed in Table 1 must vanish. For the remaining CPC, R1, and LPPD (and even LPC) cases, the normal forms derived for bifurcations of generic ODEs and given in Table 1 cannot be applied verbatim, because periodically forced systems are not generic due to the above-mentioned property of the return time, which results in a special Jordan structure of their monodromy

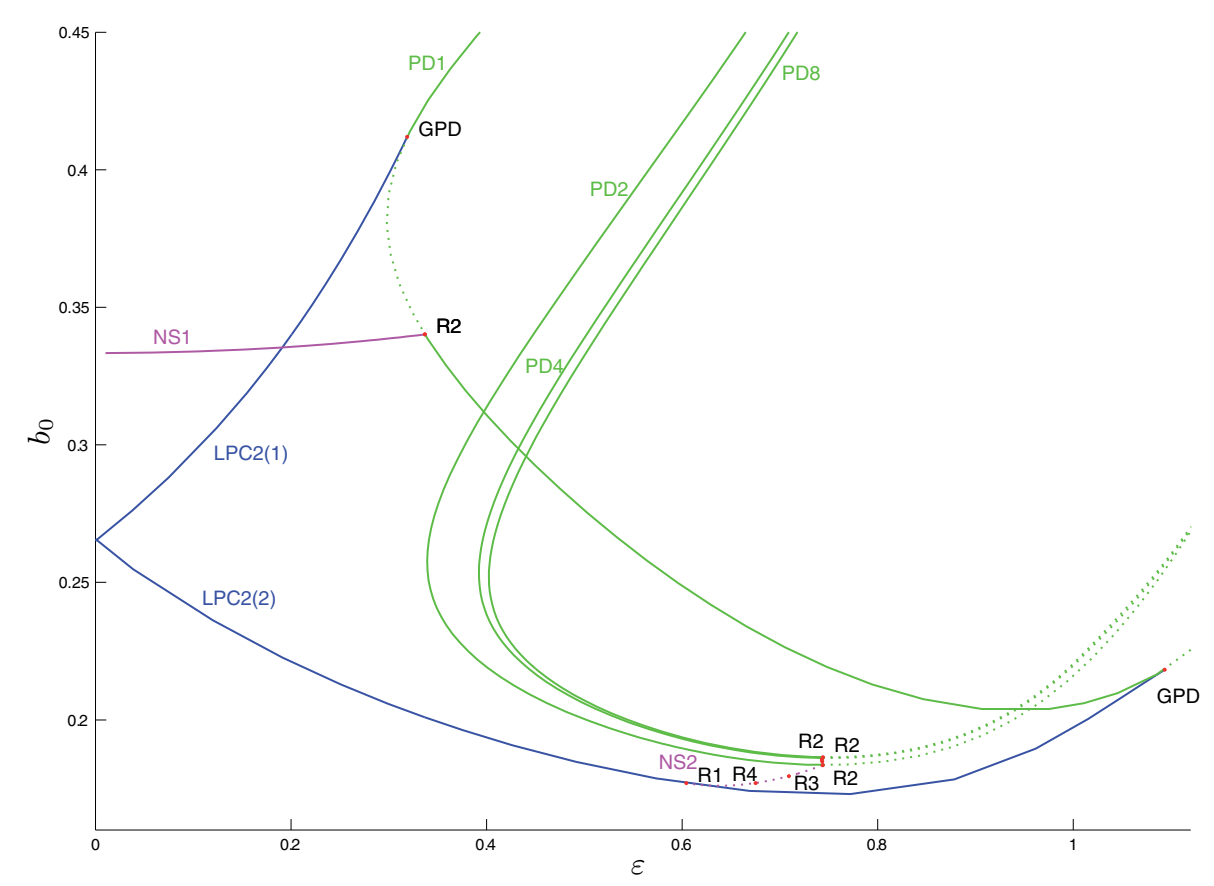

Figure 1. Bifurcation diagram of limit cycles in system (5.2). In blue are the limit points of cycle bifurcations, in green period doubling bifurcations, and in magenta Neimark-Sacker bifurcations. Solid/dotted curves correspond to supercritical/subcritical bifurcations. 
matrix. Let us illustrate this phenomenon. Consider a continuation of a period-doubled limit cycle in (5.2), and suppose that its LPC bifurcation is detected. For each point of the continuation, we compute the singular values of the monodromy matrix minus the identity matrix. The two smallest singular values are shown in Figure 2. There is always one singular value equal to zero, but also the second one vanishes when approaching the LPC point. This means that instead of a Jordan block of length two (as is expected at the LPC point in generic ODEs [25]), we have in fact two Jordan blocks of length one. Therefore, we cannot apply the general theory derived for generic LPC points. A similar situation is encountered when the original limit cycle undergoes an LPC bifurcation. Something analogous happens in the CPC, R1, and LPPD cases for the periodically forced systems, which therefore should be treated separately. Normal forms for periodically forced ODEs were studied in [19].

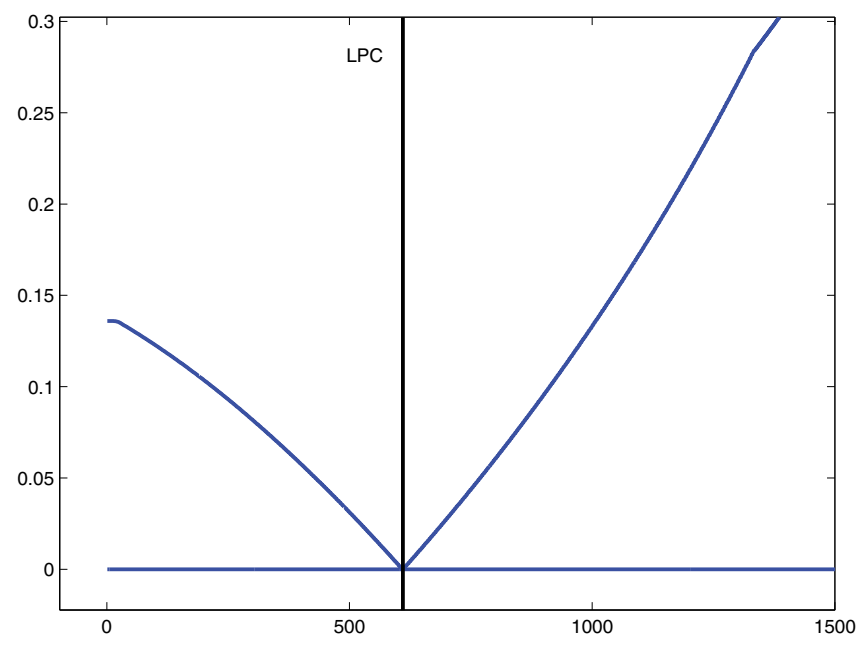

Figure 2. The two smallest singular values.

As can be seen in Figure 1, R1 points are detected in the periodically forced system. Due to the above remark, we will not attempt any normal form analysis of these points. We will analyze in detail other detected codim 2 points, reporting the normal form coefficients computed as explained in section 4 .

5.1.1. The two GPD points. In Figure 1 the LPC2 curves are tangent to the PD1 curve in two different GPD points. In the first one, with parameter values $\left(\varepsilon, b_{0}\right)=(0.319,0.412)$, the LPC curve is tangent to the subcritical period-doubling curve (type presented in Figure 9.3 of [29]), while in the second one, for $\left(\varepsilon, b_{0}\right)=(1.093,0.218)$, the LPC2 curve is tangent to the supercritical part of the PD1 bifurcation curve.

Performing the computation of the GPD normal form coefficients at the first point, we obtain the following:

- For the first equation of the GPD normal form the two coefficients $\alpha_{1}$ and $\alpha_{2}$, up to a scaling term $T$ and $T^{2}$ computed through the formula (4.14) and (4.15), are zero, up to the accuracy of the computation. 
- The normal form coefficient of the second equation, computed through formula (4.16), equals $e=-58.287$.

Notice that these results are in agreement with what we expected. Indeed, since we are in the case presented in Figure 9.3 of [29], the normal form coefficient $e$ is negative.

From the computation of the GPD normal form coefficients at the second critical point we obtain the following:

- For the first equation of the GPD normal form the two coefficients equal zero up to the accuracy of the computation.

- The value of the normal form coefficient of the second equation is $e=41.544$.

Also in this case the obtained results are in agreement with the theory.

5.1.2. The 1:2 resonance points. We divide the $1: 2$ resonance points present in this model into two groups, namely, the R2 point at $\left(\varepsilon, b_{0}\right)=(0.337,0.340)$ and the cascade of resonance points in the right lower part of the graph.

The isolated R2 point forms the intersection of the NS1 curve, the supercritical NeimarkSacker curve of a limit cycle with period approximately equal to 1, and PD1. The situation is thus the one depicted in Figure 8.8 of [29]. Performing the normal form coefficient computation we obtain the following:

- In the first equation of the R2 normal form $\alpha=0$.

- In the last equation of the R2 normal form we have $(a, b)=(3.401,-12.907)$.

Note that the obtained results are in accordance with the theory. Indeed, the absence of a secondary Neimark-Sacker curve implies that $a>0$, and the supercriticality of the NeimarkSacker curve implies that $b<0$.

In the lower right part of the bifurcation diagram a resonance cascade is present, which accumulates on the sequence of period-doubling curves. A zoom of this part is shown in Figure 3. Each resonance point of this cascade is a point of the type represented in Figure 9.10 of [29] (so with $a<0$ and the sign of $b$ dependent on the criticality of the incoming Neimark-Sacker curve). Notice that the criticality of the NS curves changes at the R2 point (as depicted in Figure 9.10 of [29]).

As a first general result we observe that, in the first equation of the R2 normal form coefficient, $\alpha=0$ for all points (as expected since the system is periodically forced). We remark that for the normal form coefficients in the $\xi$-equations of the normal forms, a computation to high accuracy is needed to get unambiguous results. The results are as follows.

On PD2. In the R2 point $\left(\varepsilon, b_{0}\right)=(0.744,0.184)$. To the left of the R2 point the PD2 curve is supercritical, and to the right it is subcritical. The NS2 curve incoming in the R2 point is subcritical, while the NS4 curve outgoing at the R2 point is supercritical. We are thus in the time reversed situation of Figure 9.10 of [29]. So we expect that $b>0$ (subcritical incoming Neimark-Sacker curve) and $a<0$ (there is an outgoing secondary Neimark-Sacker curve). The computed critical coefficients at the $\mathrm{R} 2$ point are $(a, b)=(-65.767,16.267)$.

On PD4. In the R2 point $\left(\varepsilon, b_{0}\right)=(0.743,0.186)$. To the left of the R2 point the PD4 curve is supercritical, and to the right it is subcritical. The NS4 curve incoming in the R2 point is supercritical, while the NS8 curve outgoing at the R2 point is subcritical. We are therefore in the situation depicted in Figure 9.10 of [29]. We expect that $b<0$ (supercritical incoming Neimark-Sacker curve) and $a<0$ (there is an outgoing secondary Neimark-Sacker 


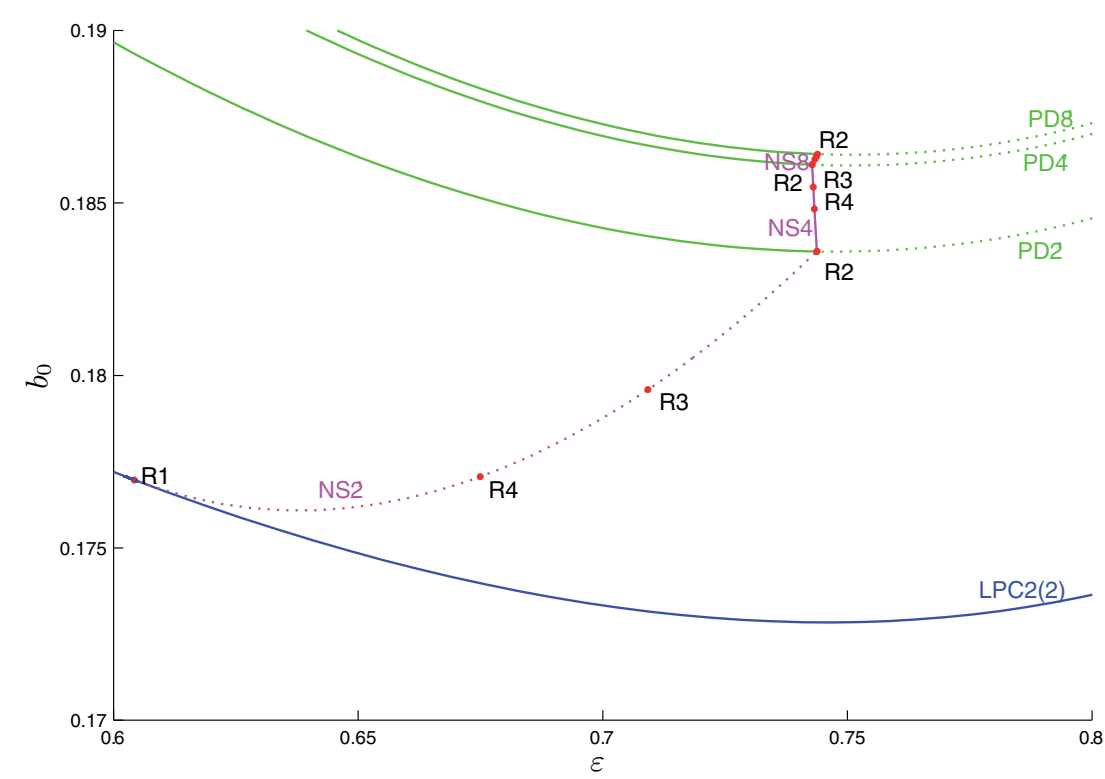

Figure 3. The resonance cascade in system (5.2). In blue are the limit points of cycle bifurcations, in green period doubling bifurcations, and in magenta Neimark-Sacker bifurcations. Solid/dotted curves correspond to supercritical/subcritical bifurcations.

curve). The computed coefficients at the $\mathrm{R} 2$ point are $(a, b)=(-269.368,-18.151)$.

On PD8. In the $\mathrm{R} 2$ point $\left(\varepsilon, b_{0}\right)=(0.744,0.186)$. To the left of the $\mathrm{R} 2$ point the $\mathrm{PD} 8$ curve is supercritical, and to the right it is subcritical. The NS8 curve incoming in the R2 point is subcritical; we are thus in the time reversed situation of Figure 9.10 of [29]. Thus, we expect that $b>0$ (subcritical incoming Neimark-Sacker curve) and $a<0$ (there is an outgoing secondary Neimark-Sacker curve, since the cascade continues). The computed coefficients of the R2 point are $(a, b)=(-921.701,16.581)$.

All the obtained results are in agreement with the theory.

5.1.3. The 1:3 resonance points. There are two 1:3 resonance points, one on NS2 and the other on NS4, as can be seen in Figure 3. These two points behave in a different way. The Neimark-Sacker curve corresponding to the first point at $\left(\varepsilon, b_{0}\right)=(0.709,0.179)$ is subcritical, so we expect $\Re(c)$ to be positive. The Neimark-Sacker curve of the second point at $\left(\varepsilon, b_{0}\right)=$ $(0.743,0.185)$ is supercritical, and so $\Re(c)$ should be negative. To check whether we are in a nondegenerate case, we also have to take $b$ into account; however, the sign of $b$ is not relevant.

- For the first R3 point we have that $(b, \Re(c))=(4.557-4.457 i, 9.155)$.

- For the second R3 point we have that $(b, \Re(c))=(0.405+12.143 i,-8.820)$.

These results are in accordance with the theory.

5.1.4. The 1:4 resonance points. There are two 1:4 resonance points, one on NS2 and the other on NS4, as can be seen in Figure 3. These two points behave in the same way as the 1:3 resonance bifurcation points. The Neimark-Sacker curve corresponding to the first point at $\left(\varepsilon, b_{0}\right)=(0.675,0.177)$ is subcritical, and so here we expect $\Re(A)$ to be positive. The Neimark-Sacker curve of the second point at $\left(\varepsilon, b_{0}\right)=(0.743,0.185)$ is supercritical, and so 
$\Re(A)$ should be negative. Moreover, since those points are part of a resonance cascade, we should not have limit point bifurcations of nontrivial equilibria, so we are in region I of Figure 9.14 of [29]. In order to ensure that we are not in a degenerate case, we also need to check that $d \neq 0$.

- For the first R4 point we have that $(c, d)=(11.624-84.897 i, 65.072+92.254 i)$, and so $A=0.103-0.752 i$.

- For the second R4 point we have that $(c, d)=(-8.580-414.721 i,-416.641-489.172 i)$, and so $A=-0.01335-0.645 i$.

The results are in accordance with the theory. For both bifurcation points the value of $A$ belongs to region I of Figure 9.14 in [29].

5.2. The Steinmetz-Larter model. The following model of the peroxidase-oxidase reaction was studied by Steinmetz and Larter [41] and is used as the test-example in [30, 21]:

$$
\left\{\begin{array}{l}
\dot{A}=-k_{1} A B X-k_{3} A B Y+k_{7}-k_{-7} A, \\
\dot{B}=-k_{1} A B X-k_{3} A B Y+k_{8} \\
\dot{X}=k_{1} A B X-2 k_{2} X^{2}+2 k_{3} A B Y-k_{4} X+k_{6} \\
\dot{Y}=-k_{3} A B Y+2 k_{2} X^{2}-k_{5} Y
\end{array}\right.
$$

where $A, B, X, Y$ are state variables and $k_{1}, k_{2}, k_{3}, k_{4}, k_{5}, k_{6}, k_{7}, k_{8}$, and $k_{-7}$ are parameters. We fix the parameters as follows.

\begin{tabular}{|l|l|l|l|l|l|l|l|}
\hline Par. & Value & Par. & Value & Par. & Value & Par. & Value \\
\hline$k_{1}$ & 0.1631021 & $k_{2}$ & 1250 & $k_{3}$ & 0.046875 & $k_{4}$ & 20 \\
$k_{5}$ & 1.104 & $k_{6}$ & 0.001 & $k_{-7}$ & 0.1175 & & \\
\hline
\end{tabular}

We perform a bifurcation analysis in the remaining parameter space $\left(k_{7}, k_{8}\right)$. A few curves are reported in Figure 4.

5.2.1. The 1:1 resonance points. The two 1:1 resonance points behave differently, since in one R1 point the Neimark-Sacker curve rooted at the bifurcation point is supercritical, while in the other one it is subcritical.

- For the R1 point in $\left(k_{7}, k_{8}\right)=(1.180,0.724)$, the two coefficients of the last equation of the $\mathrm{R} 1$ normal form are equal to $(a, b)=\left(-3.65410^{-3}, 0.735\right)$. Their product $a b=-2.68610^{-3}$ is negative, which corresponds to the fact that the Neimark-Sacker curve rooted at the $\mathrm{R} 1$ point is supercritical.

- For the $\mathrm{R} 1$ point in $\left(k_{7}, k_{8}\right)=(1.858,0.930)$, the two coefficients of the last equation of the $\mathrm{R} 1$ normal form are equal to $(a, b)=\left(-6.64310^{-2},-2.157\right)$. Their product $a b=0.143$ is positive, and indeed the Neimark-Sacker curve rooted at the R1 point is subcritical.

So we can conclude that the results are in accordance with the theory.

5.2.2. The $\mathbf{C H}$ points. In Figure 4 we see a $\mathrm{CH}$ point at $\left(k_{7}, k_{8}\right)=(1.757,0.913)$. The normal form coefficient at that bifurcation point equals $\Re(e)=1.392$ and is hence positive. In order to verify that the normal form computation is correct, one might use tori continuation techniques [38, 27, 11, 16, 35, 39]. However, these techniques are not stable near critical cases like the one we have. In order to validate our result we therefore rely on simulations. 


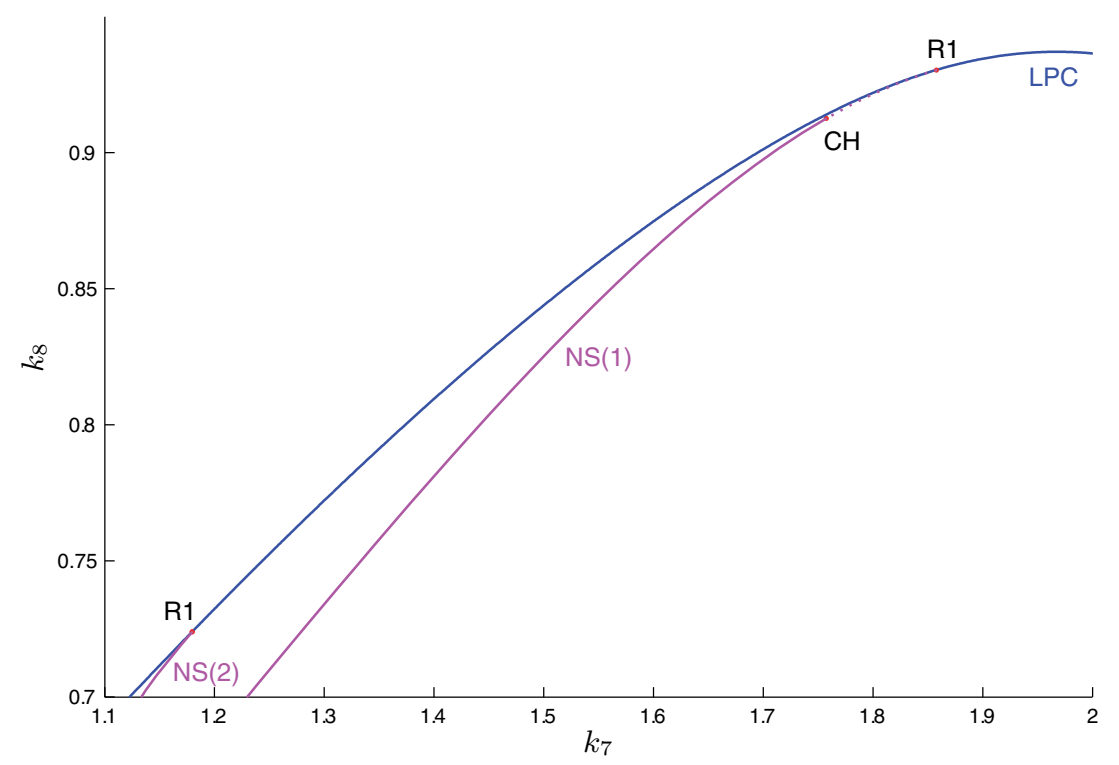

Figure 4. Bifurcation diagram of limit cycles in model (5.3). In blue are the limit points of cycle bifurcations and in magenta Neimark-Sacker bifurcations. Solid/dotted curves correspond to supercritical/subcritical bifurcations.

The obtained result is shown in Figure 5. The indicated regions correspond with the regions as denoted in [29]. The magenta curve between regions 2 and 3 is the supercritical Neimark-Sacker curve, and the red curve between regions 1 and 2 is the subcritical NeimarkSacker curve. For each point of the grid, we have started time integration from a point close to the original limit cycle (a 1\% perturbation) until an attractor was found. The 1-norm of the $X$-coordinate of an orbit with time length 1000 along the attractor is shown in the colormap. In region 2 this attractor is the original limit cycle, and in region 3 it is the inner torus arisen through the supercritical Neimark-Sacker curve. In region 1 the original limit cycle is unstable, and so the trajectory which starts nearby converges to another attractor. Between regions 1 and 3 and regions 1 and 2 a catastrophic bifurcation happens, i.e., a drastic change of the attractor, identified from the change of color which varies from blue to red. Right above the $\mathrm{CH}$ point, the catastrophic bifurcation is the subcritical Neimark-Sacker curve, while left below it is the limit point of tori $\left(T_{c}\right)$ curve. Figure 5 shows that we obtain the scenario which corresponds to a positive second Lyapunov coefficient.

5.3. The Lorenz-84 system. This model, taken from [36], is a meteorological model proposed by Lorenz in 1984 in order to describe the atmospheric circulation. The equations of the model are

$$
\left\{\begin{array}{l}
\dot{x}=-y^{2}-z^{2}-a x+a F, \\
\dot{y}=x y-b x z-y+G, \\
\dot{z}=b x y+x z-z,
\end{array}\right.
$$

where $(a, b, F, G)$ are parameters. We fix $a=0.25$ and $b=4$. This model, as found in $[40,43]$, has most of the analyzed codim 2 bifurcations of limit cycles. We report in Figure 6 a 




Figure 5. Simulations on a parameter grid (black points) of system (5.3). The magenta solid/dotted line is the supercritical/subcritical Neimark-Sacker curve. The color represents the value of the maximum of the first coordinate of the attractor reached through simulation from a point close to the limit cycle.

bifurcation diagram recomputed and extended with MATCONT in which the bifurcations of equilibria (LP stands for limit point and $\mathrm{H}$ for Andronov-Hopf) are thicker and the limit cycle bifurcations are thin. In particular, the blue curve is an LPC bifurcation curve, the green curves are period-doubling (PD) bifurcation curves, and the magenta curves are NeimarkSacker (NS) curves. The codim 2 points are marked with a red dot, and, as can be seen in the figure, almost all cases, except for the $\mathrm{CH}$ bifurcation and the fold-flip bifurcation, are present in this model. In the remainder of this section we will investigate the normal form coefficients of each bifurcation.

5.3.1. The swallow-tail bifurcation. The first degeneracy we want to analyze is the vanishing of the coefficient $c$ in the CPC normal form. This bifurcation, named the swallow-tail bifurcation, is in our case characterized by the collision and disappearance of two cusp points of limit cycles. In order to get this codimension three (codim 3) bifurcation we analyze part of the blue curve in Figure 6 for different parameter values of $b$. The result is shown in Figure 7. Part of the LPC branch is plotted in the $(G, F)$-plane for different values of parameter $b \in[2.91,2.95]$ (from blue to red). In the table we can see the behavior of the critical normal form coefficient $c$, where it exists (the colors correspond to those from the bifurcation diagram). Notice how the behavior of this codim 3 bifurcation is captured by a smooth vanishing of the normal form coefficient. 


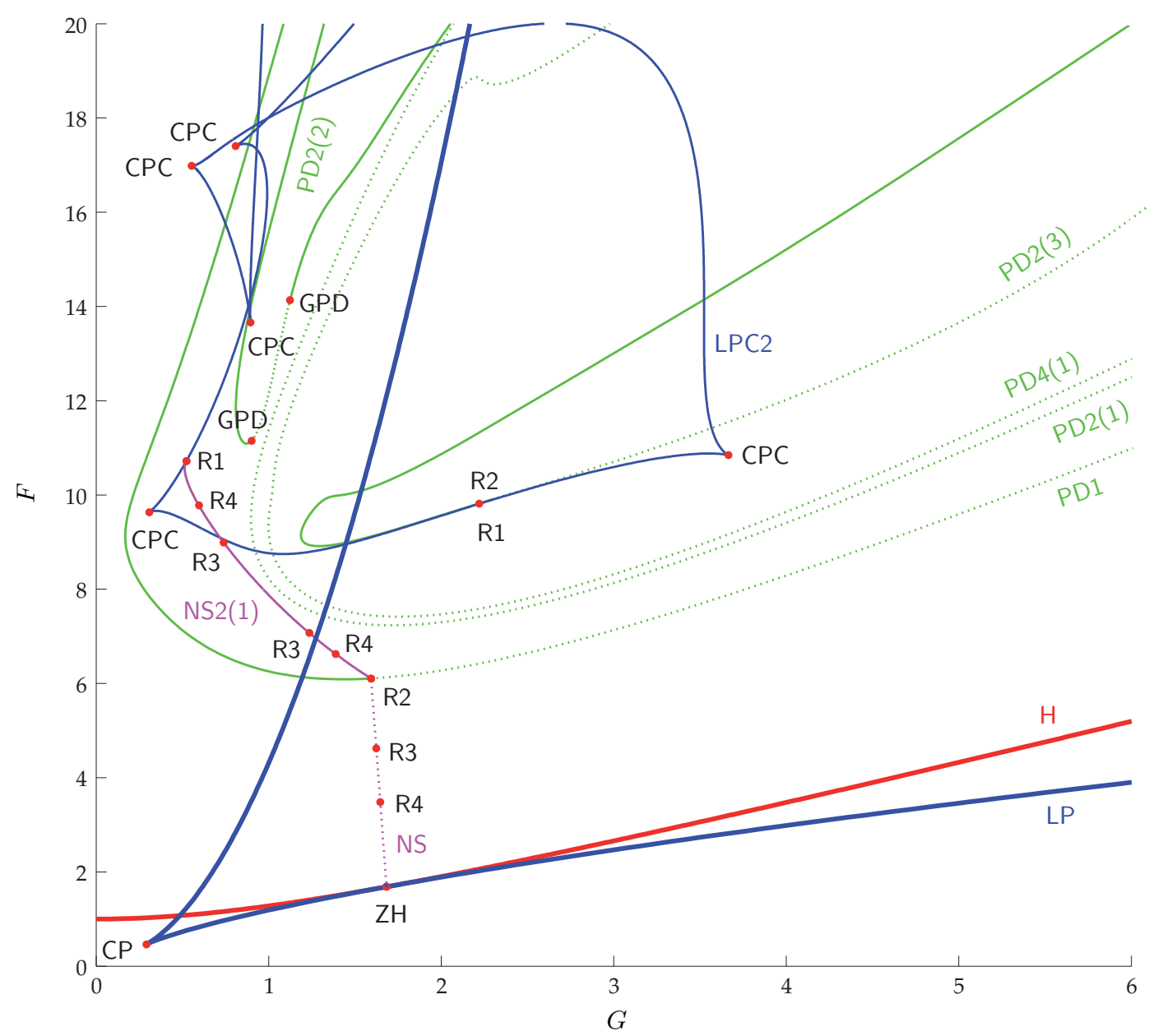

Figure 6. Bifurcation diagram of model (5.4). The thicker curves are bifurcation curves of equilibria; the thin curves are bifurcation curves of limit cycles and invariant tori (in blue the limit points of cycle curves, in green period doubling curves, and in magenta Neimark-Sacker curves). Solid/dotted curves correspond to supercritical/subcritical bifurcations.

5.3.2. The degenerate GPD bifurcation. On the green curve PD2(2) of Figure 6 there are two GPD points. Computing the normal form coefficient in the first GPD point, with parameter values $(G, F)=(0.900,11.145)$, gives $e=-1.31810^{-3}<0$. Therefore, there is an LPC bifurcation curve that starts rightward tangent to the subcritical part of the perioddoubling manifold. In the second case, namely, for $(G, F)=(1.124,14.129), e=2.89510^{-3}>$ 0 , and so the LPC curve starts rightward tangent to the supercritical part of the PD curve. These conclusions are clarified in Figure 8; in the upper panels the Poincaré maps of the limit cycles involved in the bifurcation are sketched. On the LPC4(2) curve the limit cycles sketched in blue and red collide and disappear, while on the LPC4(1) curve the two involved limit cycles are sketched in red and green. 


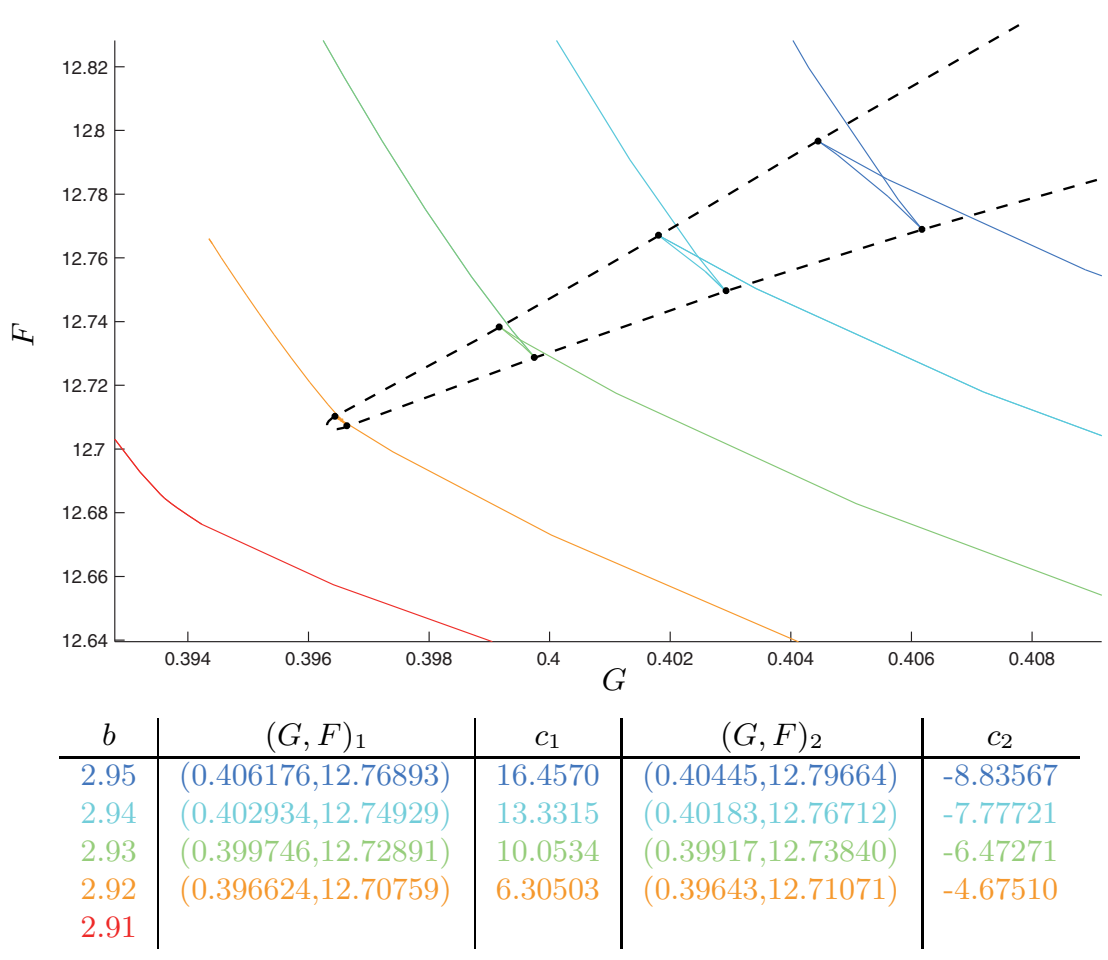

Figure 7. Different limit points of cycle bifurcation curves in the $(G, F)$-plane for different values of the third parameter $b$. The parameter values are reported in the table.

5.3.3. The 1:1 resonance points. Two R1 points are located on the LPC curve. Those two points should have different products of normal form coefficients. In fact, in the first one, where $(G, F)=(0.522,10.718)$, the Neimark-Sacker curve rooted at the bifurcation point is supercritical (i.e., the situation depicted in Figure 8.8 of [29]), while in the second one, where $(G, F)=(2.220,9.811)$, the Neimark-Sacker curve is subcritical.

- For the first $\mathrm{R} 1$ point $(a, b)=(2.577,-1.266)$, so the product $a b=-3.262$ is negative.

- For the second R1 point $(a, b)=(-9.887,-2.005)$, so the product $a b=19.819$ is positive.

These results are in accordance with the theory.

5.3.4. The 1:2 resonance points. At the $\mathrm{R} 2$ point at $(G, F)=(1.593,6.106)$ shown in Figure 6, the incoming Neimark-Sacker curve NS is subcritical (therefore, we must have $b>0$ ), while the outgoing curve NS2 (which exists and thus $a<0$ ) is supercritical; i.e., we are in the time reversed case of Figure 9.10 of [29]. The coefficients computed at the 1:2 resonance point are $(a, b)=(-0.633,0.179)$, in accordance with the theory.

At the $\mathrm{R} 1$ point located at $(G, F)=(2.220,9.811)$ starts a resonance $1: 2$ cascade, as shown in Figure 9. On the cascade we find many resonance points which we will analyze in what follows. In particular, since the R2 points belong to a cascade, they are of the type presented in Figure 9.10 of [29] (so $a<0$ ), with at each step a change of criticality of the incoming Neimark-Sacker curve. The Neimark-Sacker curve born at the R1 point is subcritical, so for the first R2 point we expect that $b>0$, while for the second one $b<0$. 


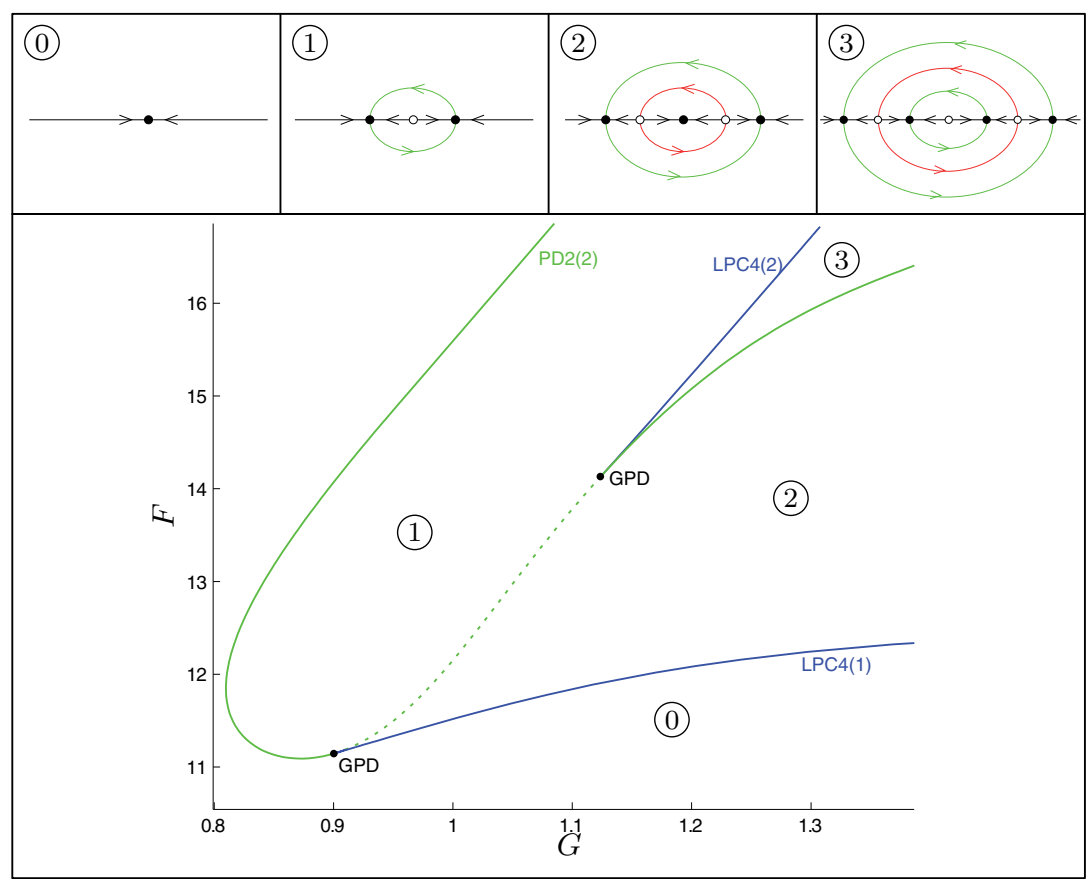

Figure 8. Two generalized period-doubling points with different normal form coefficients on the perioddoubling bifurcation curve PD2(2) of Figure 6.

- For the first R2 point at $(G, F)=(2.298,9.916)$ we have that $(a, b)=(-1.316,0.111)$.

- For the second R2 point at $(G, F)=(2.298,9.920)$ we have that $(a, b)=(-2.623$, $\left.-5.64110^{-2}\right)$.

Results are in accordance with the theory.

5.3.5. The 1:3 resonance points. There are several 1:3 resonance points at which we can have a closer look. There is one R3 point located on the NS curve and two R3 points are detected on the NS2 curve. The R3 bifurcation corresponding to the first iterate happens at $(G, F)=(1.624,4.628)$, with a positive normal form coefficient of the Neimark-Sacker bifurcation. The R3 points corresponding to the second iterate are at $(G, F)=(1.235,7.072)$ and $(G, F)=(0.739,8.989)$, where the Neimark-Sacker bifurcation is in both cases supercritical, so we are in the situation depicted in Figure 9.12 of [29].

- For the R3 point at $(G, F)=(1.624,4.628)$ we have that $(b, \Re(c))=(0.191-0.546 i$, $\left.6.18610^{-2}\right)$.

- For the R3 point at $(G, F)=(1.235,7.072)$ we have that $(b, \Re(c))=(-0.446-$ $\left.0.190 i,-3.61210^{-2}\right)$.

- For the R3 point at $(G, F)=(0.7394,8.989)$ we have that $(b, \Re(c))=(-0.129+$ $\left.1.68110^{-2} i,-1.95110^{-2}\right)$.

All these results are in accordance with the theory.

There are also R3 points on the cascade, shown in Figure 9. The first one corresponds with a subcritical Neimark-Sacker curve, while the second one corresponds with a supercritical 


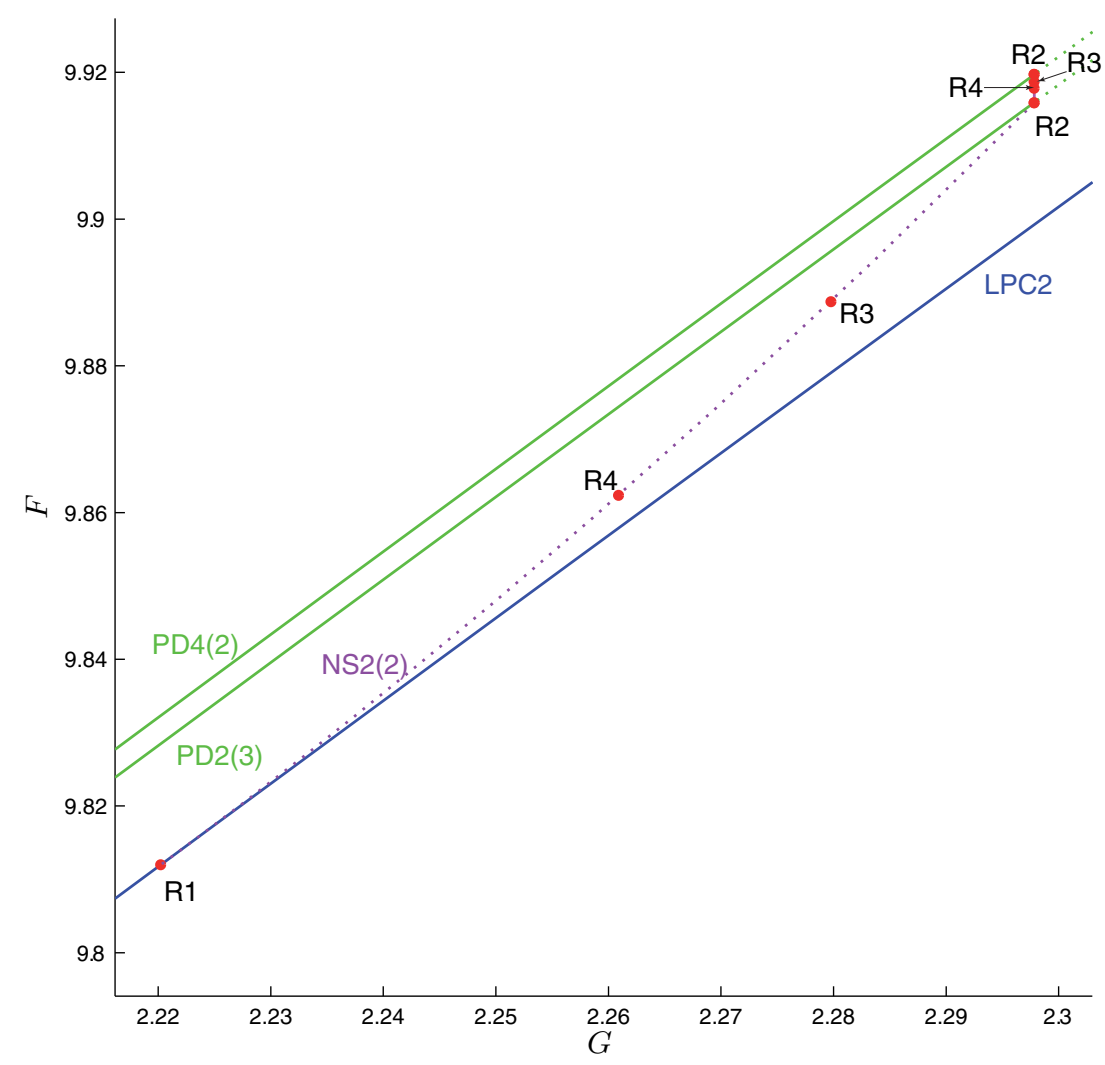

Figure 9. Zoom on the resonance 1: 2 cascade that starts at the right $\mathrm{R} 1$ point in Figure 6 . In blue are the limit points of cycle bifurcation curves, in green the period-doubling curves, and in violet the Neimark-Sacker curves. Solid/dotted curves correspond to supercritical/subcritical curves.

Neimark-Sacker curve.

- For the first R3 point, at $(G, F)=(2.279,9.889)$ we have that $(b, \Re(c))=(-2.958-$ $0.360 i, 0.738)$.

- For the second R3 point, at $(G, F)=(2.297,9.919)$ we have that $(b, \Re(c))=(2.745+$ $3.539 i,-0.385)$.

Also in this case all results are in accordance with the theory.

5.3.6. The 1:4 resonance points. There are five 1:4 resonance points at which we will have a closer look. One is located on the NS curve, two others are on the NS2 curve, and the last two lie on the resonance cascade (see Figure 9).

- For the R4 point at $(G, F)=(1.647,3.376)$ we have that $(c, d)=\left(5.004510^{-2}-\right.$ $7.45910^{-2} i, 0.110+0.534 i$ ), and so $A=9.17910^{-2}-0.137 i$ (subcritical NeimarkSacker curve, case I).

- For the R4 point at $(G, F)=(0.595,9.777)$ we have that $(c, d)=\left(-1.51310^{-2}-\right.$ $0.135 i,-2.66510^{-2}-4.11210^{-2} i$ ), and so $A=-0.308-2.753 i$ (supercritical NeimarkSacker curve, case VIII).

- For the R4 point at $(G, F)=(1.390,6.620)$ we have that $(c, d)=\left(-4.17210^{-2}-\right.$ 
$0.992 i,-0.428-1.082 i$ ), and so $A=-3.584-0.852 i$ (supercritical Neimark-Sacker curve, case I).

For the first and the last points no further bifurcation analysis is possible to confirm the correctness of the results since the curves rooted at the bifurcation point are global bifurcations of limit cycles. Instead, it is possible to continue all local bifurcations of limit cycles rooted at the second R4 point, obtaining the result shown in Figure 10. The curve $T^{\text {in }}$ is shown in the bifurcation diagram of the R4 point, given in [29]. These diagrams are also presented in [13]. Curve $T$ corresponds to the fold bifurcation of the $4 T$-periodic cycle, which happens in the "big" cycle. For curve $T^{i n}$, the fold bifurcation happens in the "big" cycle. Note that we have not made the distinction between regions VII and VIII.

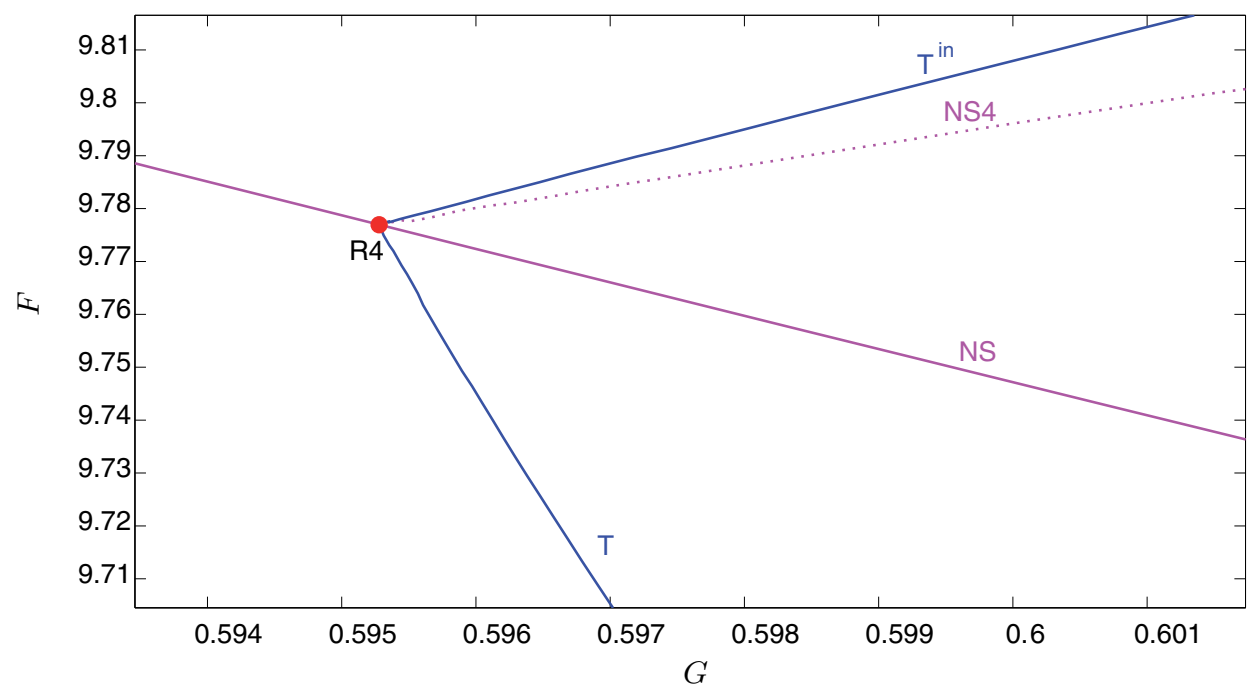

Figure 10. Bifurcation diagram at the $\mathrm{R} 4$ point at $(G, F)=(0.595,9.777)$. In blue are the limit points of cycle bifurcation curves and in violet the Neimark-Sacker curves. Solid/dotted curves correspond to supercritical/subcritical curves.

The first R4 point of the resonance cascade lies on a subcritical Neimark-Sacker curve, while the second one lies on a supercritical Neimark-Sacker curve. Moreover, since they are part of a cascade, we expect them to be of type I.

- For the first R4 point at $(G, F)=(2.298,9.916)$ we have that $(c, d)=\left(5.18510^{-2}-\right.$ $1.763 i,-2.014+0.455 i)$, and so $A=2.51010^{-2}-0.854 i$.

- For the first R4 point at $(G, F)=(2.298,9.919)$ we have that $(c, d)=\left(-2.82110^{-2}-\right.$ $6.815 i,-10.845+2.146 i)$, and so $A=-2.55010^{-3}-0.616 i$.

Also in this case the results are in accordance with the theory.

5.4. The extended Lorenz-84 system. As done in [33], it is possible to extend the Lorenz84 system (5.4) by adding a fourth variable which takes the influence on the jet stream and the baroclinic waves of external parameters like the temperature of the sea surface into account. 
The resulting system is

$$
\left\{\begin{array}{l}
\dot{x}=-y^{2}-z^{2}-a x+a F-\gamma u^{2} \\
\dot{y}=x y-b x z-y+G \\
\dot{z}=b x y+x z-z \\
\dot{u}=-\delta u+\gamma u x+K
\end{array}\right.
$$

We use the parameter values mentioned in [33], i.e.,

$$
a=0.25, b=1, G=0.2, \delta=1.04, \gamma=0.987, F=1.75, K=0.0003 .
$$

Time integrating this system from the origin leads to the detection of a stable limit cycle. In a continuation with $K$ as free system parameter the limit cycle undergoes a supercritical period-doubling bifurcation. Now, we can perform a two-parameter continuation in $(F, K)$ and obtain the bifurcation diagram reported in Figure 11 (cf. [33]).

5.4.1. The fold-flip point. As can be seen in Figure 11, a fold-flip point is detected for $(F, K)=\left(1.762,2.80610^{-4}\right)$. Since there is a Neimark-Sacker curve of the period-doubled limit cycle rooted at the bifurcation point and the NS2 curve and the LPC curve lie on different sides of the PD curve, we are in the situation represented in Figure 9.25 of [29]; i.e., we have $a_{20} b_{11}<0$ and $a_{02} b_{11}<0$. Moreover, since the NS2 curve is supercritical, $C_{N S}$ should be negative. Numerically, we obtain that $b_{11}=562.222, a_{20}=-0.576, a_{02}=$ $-1.78410^{-5}, C_{N S}=-1.07610^{7}$. Hence, these results are in agreement with the theory. They also agree with [33], where the LPPD bifurcation was analyzed by computing the normal form



Figure 11. Bifurcation diagram of limit cycles in model (5.5). The blue curve is a limit point of the cycle curve, the green one is a period-doubling curve (solid/dotted parts correspond to supercritical/subcritical parts), and the magenta curve is a supercritical Neimark-Sacker bifurcation curve of the period-doubled limit cycle. 
coefficients for the critical Poincaré map, using the numerical integration of the variational equations to compute the multilinear forms in the Taylor expansion of this map.

6. Discussion. In this paper, we have applied the approach from [30] to codim 2 bifurcations of limit cycles in generic autonomous ODE systems (1.1). This approach is based on the periodic normalization by Iooss [25]; see also [26].

Although in $[10,25]$ periodic normal forms for some codim 2 bifurcations of limit cycles were presented, neither of these publications treated all 11 codim 2 local bifurcations of limit cycles. We derived the normal forms for eight cases when the center manifold is either two- or three-dimensional. Moreover, we have provided the explicit formulas for the normal form coefficients. These formulas are directly suitable for numerical implementation using orthogonal collocation. They perfectly fit into the continuation context, where limit cycles and their bifurcations are computed using the BVP approach, without numerical approximation of Poincaré maps. Full details of the implementation of the developed method in MATCONT were given, together with several numerical examples.

The three remaining codim 2 cases with four- and five-dimensional center manifolds, i.e., fold-NS (LPNS), period doubling-NS (PDNS), and double NS (NSNS) bifurcations, will be treated in a separate paper.

Acknowledgments. The authors would like to thank H. G. E. Meijer (University of Twente, The Netherlands) for useful discussions. The authors would also like to thank two referees whose reviews led to a substantial improvement of the presentation and even the content of the paper.

\section{REFERENCES}

[1] A. Abad, R. Barrio, F. Blesa, M. Rodriguez, and the Grupo Mecánica Espacial, tideS: $A$ Taylor Integrator for Differential Equations, http://gme.unizar.es/software/tides.

[2] V. I. ARnol'D, Geometrical Methods in the Theory of Ordinary Differential Equations, Springer-Verlag, New York, 1983.

[3] V. I. Arnol'd, V. S. Afrajmovich, Yu. S. Il'yashenko, and L. P. Shil'nikov, Dynamical Systems $V$, Bifurcation Theory and Catastrophe Theory, Encyclopaedia of Mathematical Sciences 5, SpringerVerlag, Berlin, 1994.

[4] D. K. Arrowsmith and C. M. Place, An Introduction to Dynamical Systems, Cambridge University Press, Cambridge, UK, 1990.

[5] U. M. Ascher, R. M. M. Mattheij, And R. D. Russell, Numerical Solution of Boundary Value Problems for Ordinary Differential Equations, Classics in Appl. Math. 13, SIAM, Philadelphia, 1995.

[6] R. BARrIo, Sensitivity analysis of ODEs/DAEs using the Taylor series method, SIAM J. Sci. Comput., 27 (2006), pp. 1929-1947.

[7] W. J. Beyn, A. Champneys, E. J. Doedel, W. Govaerts, Yu. A. Kuznetsov, and B. Sandstede, Numerical continuation and computation of normal forms, in Handbook of Dynamical Systems, Vol. 2, 3rd ed., B. Fiedler, ed., AMS, Providence, RI, 2002, pp. 149-219.

[8] H. Broer, C. Simó, ANd R. Vitolo, Bifurcations and strange attractors in the Lorenz-84 climate model with seasonal forcing, Nonlinearity, 15 (2002), pp. 1205-1267.

[9] M. Capinski, Z. Galias, T. Kapela, M. Mrozek, P. Pilarczyk, D. Wilczak, and P. Zgliczyński, CAPD: Computer Assisted Proofs in Dynamics, http://capd.ii.uj.edu.pl.

[10] S.-N. Chow And D. WAng, Normal forms of bifurcating periodic orbits, in Multiparameter Bifurcation Theory (Arcata, CA, 1985), Contemp. Math. 56, AMS, Providence, RI, 1986, pp. 9-18. 
[11] H. Dankowicz and G. Thakur, A Newton method for locating invariant tori of maps, Internat. J. Bifur. Chaos Appl. Sci. Engrg., 16 (2006), pp. 1491-1503.

[12] C. De Boor And B. Swartz, Collocation at Gaussian points, SIAM J. Numer. Anal., 10 (1973), pp. 582606.

[13] F. Della Rossa, V. De Witte, W. Govaerts, and Yu. A. Kuznetsov, Numerical periodic normalization for codim 2 bifurcations of limit cycles, preprint, http://arxiv.org/abs/1111.4445, 2011.

[14] A. Dhooge, W. Govaerts, and Yu. A. Kuznetsov, MATCONT: A MATLAB package for numerical bifurcation analysis of ODEs, ACM Trans. Math. Software, 29 (2003), pp. 141-164. http://sourceforge. net/projects/matcont.

[15] A. Dhooge, W. Govaerts, Yu. A. Kuznetsov, W. Mestrom, and A. M. Riet, CL_matcont: A continuation toolbox in MATLAB, in Symposium on Applied Computing (Melbourne, FL), ACM, New York, 2003, pp. 161-166.

[16] L. Dieci, J. Lorenz, And R. D. Russell, Numerical calculation of invariant tori, SIAM J. Sci. Statist. Comput., 12 (1991), pp. 607-647.

[17] E. J. Doedel, A. R. Champneys, T. F. Fairgrieve, Yu. A. Kuznetsov, B. Sandstede, And X. J. WANG, AUTO97: Continuation and Bifurcation Software for Ordinary Differential Equations (with HomCont), http://cmvl.cs.concordia.ca/auto, 1997.

[18] E. J. Doedel, W. Govaerts, and Yu. A. Kuznetsov, Computation of periodic solution bifurcations in ODEs using bordered systems, SIAM J. Numer. Anal., 41 (2003), pp. 401-435.

[19] C. Elphick, G. Iooss, AND E. TiRAPEgui, Normal form reduction for time-periodically driven differential equations, Phys. Lett. A, 120 (1987), pp. 459-463.

[20] W. Govaerts, R. Khoshsiar Ghaziani, Yu. A. Kuznetsov, and H. G. E. Meijer, Numerical methods for two-parameter local bifurcation analysis of maps, SIAM J. Sci. Comput., 29 (2007), pp. 2644-2667.

[21] W. Govaerts, Yu. A. Kuznetsov, And A. Dhooge, Numerical continuation of bifurcations of limit cycles in MATLAB, SIAM J. Sci. Comput., 27 (2005), pp. 231-252.

[22] A. Griewank, D. Juedes, And J. Utke, Algorithm 755: ADOL-C: A package for the automatic differentiation of algorithms written in $\mathrm{C} / \mathrm{C}++$, ACM Trans. Math. Software, 22 (1996), pp. 131167 .

[23] J. GuCKenheimer And B. Meloon, Computing periodic orbits and their bifurcations with automatic differentiation, SIAM J. Sci. Comput., 22 (2000), pp. 951-985.

[24] G. Iooss, Bifurcation of Maps and Applications, North-Holland Math. Stud. 36, North-Holland, Amsterdam, 1979.

[25] G. Iooss, Global characterization of the normal form for a vector field near a closed orbit, J. Differential Equations, 76 (1988), pp. 47-76.

[26] G. Iooss And M. Adelmeyer, Topics in Bifurcation Theory and Applications, Adv. Ser. Nonlinear Dynam. 3, World Scientific, River Edge, NJ, 1992.

[27] I. G. Kevrekidis, R. Aris, L. D. Schmidt, and S. Pelikan, Numerical computation of invariant circles of maps, Phys. D, 16 (1985), pp. 243-251.

[28] B. Krauskopf, Bifurcation sequences at 1 : 4 resonance: An inventory, Nonlinearity, 7 (1994), pp. 10731091.

[29] Yu. A. Kuznetsov, Elements of Applied Bifurcation Theory, 3rd ed., Springer-Verlag, New York, 2004.

[30] Yu. A. Kuznetsov, W. Govaerts, E. J. Doedel, And A. Dhooge, Numerical periodic normalization for codim 1 bifurcations of limit cycles, SIAM J. Numer. Anal., 43 (2005), pp. 1407-1435.

[31] Yu. A. Kuznetsov and V. V. Levitin, CONTENT: A multiplatform environment for analyzing dynamical systems, 1997.

[32] Yu. A. Kuznetsov and H. G. E. MeiJer, Numerical normal forms for codim 2 bifurcations of fixed points with at most two critical eigenvalues, SIAM J. Sci. Comput., 26 (2005), pp. 1932-1954.

[33] Yu. A. Kuznetsov, H. G. E. Meijer, and L. van Veen, The fold-flip bifurcation, Internat. J. Bifur. Chaos Appl. Sci. Engrg., 14 (2004), pp. 2253-2282.

[34] Yu. A. Kuznetsov, S. Muratori, And S. Rinaldi, Bifurcations and chaos in a periodic predator-prey model, Internat. J. Bifur. Chaos Appl. Sci. Engrg., 2 (1992), pp. 117-128.

[35] Y. Lan, C. Chandre, And P. Cvitanovic, Newton's descent method for the determination of invariant tori, Phys. Rev. E, 74 (2006), 046206. 
[36] E. N. LoRenz, Irregularity: A fundamental property of the atmosphere, Tellus, 36 (1984), pp. 98-110.

[37] B. B. Peckham and I. G. Kevrekidis, Period doubling with higher-order degeneracies, SIAM J. Math. Anal., 22 (1991), pp. 1552-1574.

[38] B. Rasmussen, Numerical Methods for the Continuation of Invariant Tori, ProQuest LLC, Ann Arbor, MI, 2003.

[39] B. Rasmussen And L. Dieci, A geometrical method for the approximation of invariant tori, J. Comput. Appl. Math., 216 (2008), pp. 388-412.

[40] A. Shilnikov, G. Nicolis, AND C. Nicolis, Bifurcation and predictability analysis of a low-order atmospheric circulation model, Internat. J. Bifur. Chaos Appl. Sci. Engrg., 5 (1995), pp. 1701-1711.

[41] C. G. Steinmetz And R. LARTer, The quasiperiodic route to chaos in a model of the peroxidase-oxidase reaction, J. Chem. Phys., 94 (1991), pp. 1388-1396.

[42] G. Torrini, R. Genesio, And A. Tesi, On the computation of characteristic multipliers for predicting limit cycle bifurcations, Chaos Solitons Fractals, 9 (1998), pp. 121-133.

[43] L. VAN VeEn, Baroclinic flow and the Lorenz-84 model, Internat. J. Bifur. Chaos Appl. Sci. Engrg., 13 (2003), pp. 2117-2139. 